\title{
The SAMI Galaxy Survey: Quenching of Star Formation in Clusters I. Transition Galaxies
}

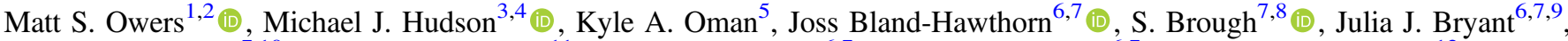 \\ Luca Cortese $^{7,10}$ (D), Warrick J. Couch ${ }^{11}$ (D), Scott M. Croom ${ }^{6,7}$, Jesse van de Sande ${ }^{6,7}$ (D), Christoph Federrath ${ }^{12}$ (iD), \\ Brent Groves $^{7,12}$ (D) A. M. Hopkins ${ }^{13}$ (ID), J. S. Lawrence ${ }^{13}$, Nuria P. F. Lorente ${ }^{13}$, Richard M. McDermid ${ }^{1,2}$, \\ Anne M. Medling ${ }^{12,14,18}$ (D), Samuel N. Richards ${ }^{15}$ (D), Nicholas Scott ${ }^{6,7}$ (D), Dan S. Taranu ${ }^{10,16}$ (D), Charlotte Welker ${ }^{10}$, and \\ Sukyoung K. $\mathrm{Yi}^{17}$ (iD \\ ${ }^{1}$ Department of Physics and Astronomy, Macquarie University, NSW, 2109, Australia; matt.owers@mq.edu.au \\ ${ }^{2}$ Astronomy, Astrophysics and Astrophotonics Research Centre, Macquarie University, Sydney, NSW 2109, Australia \\ 3 Department of Physics \& Astronomy, University of Waterloo, Waterloo, ON, N2L 3G1, Canada \\ ${ }^{4}$ Perimeter Institute for Theoretical Physics, 31 Caroline St.N., Waterloo, ON, N2L 2Y5, Canada \\ ${ }^{5}$ Kapteyn Astronomical Institute, University of Groningen, Postbus 800, NL-9700 AV Groningen, The Netherlands \\ ${ }^{6}$ Sydney Institute for Astronomy (SIfA), School of Physics, The University of Sydney, NSW, 2006, Australia \\ ${ }^{7}$ ARC Centre of Excellence for All Sky Astrophysics in 3 Dimensions (ASTRO 3D), Australia \\ ${ }^{8}$ School of Physics, University of New South Wales, NSW 2052, Australia \\ ${ }^{9}$ Australian Astronomical Optics, AAO-USydney, School of Physics, University of Sydney, NSW 2006, Australia \\ Australian Astronomical Optics, AAO-USydney, School of Physics, University of Sydney, NSW 2006, Australia
${ }^{10}$ International Centre for Radio Astronomy Research, University of Western Australia, 35 Stirling Highway, Crawley WA 6009, Australia \\ ${ }^{11}$ Centre for Astrophysics and Supercomputing, Swinburne University of Technology, VIC 3122, Melbourne, Australia \\ ${ }^{12}$ Research School for Astronomy \& Astrophysics, Australian National University, Canberra, ACT 2611, Australia \\ ${ }^{13}$ Australian Astronomical Optics, Macquarie University, 105 Delhi Rd, North Ryde, NSW 2113, Australia \\ ${ }^{14}$ Ritter Astrophysical Research Center, University of Toledo, Toledo, OH 43606, USA \\ ${ }^{15}$ SOFIA Science Center, USRA, NASA Ames Research Center, Building N232, M/S 232-12, P.O. Box 1, Moffett Field, CA 94035-0001, USA \\ ${ }^{16}$ Department of Astrophysical Sciences, Princeton University, 4 Ivy Lane, Princeton, NJ 08544, USA \\ ${ }^{17}$ Department of Astronomy and Yonsei University Observatory, Yonsei University, Seoul 03722, Republic of Korea \\ Received 2018 December 13; revised 2019 January 21; accepted 2019 January 23; published 2019 March 4
}

\begin{abstract}
We use integral-field spectroscopy from the SAMI Galaxy Survey to identify galaxies that show evidence of recent quenching of star formation. The galaxies exhibit strong Balmer absorption in the absence of ongoing star formation in more than $10 \%$ of their spectra within the SAMI field of view. These H $\delta$-strong (HDS) galaxies (HDSGs) are rare, making up only $\sim 2 \%(25 / 1220)$ of galaxies with stellar mass $\log \left(M_{*} / M_{\odot}\right)>10$. The HDSGs make up a significant fraction of nonpassive cluster galaxies $(15 \% ; 17 / 115)$ and a smaller fraction $(2.0 \% ; 8 / 387)$ of the nonpassive population in low-density environments. The majority (9/17) of cluster HDSGs show evidence of star formation at their centers, with the HDS regions found in the outer parts of the galaxy. Conversely, the HDS signal is more evenly spread across the galaxy for the majority (6/8) of HDSGs in low-density environments and is often associated with emission lines that are not due to star formation. We investigate the location of the HDSGs in the clusters, finding that they are exclusively within $0.6 R_{200}$ of the cluster center and have a significantly higher velocity dispersion relative to the cluster population. Comparing their distribution in projected phase space to those derived from cosmological simulations indicates that the cluster HDSGs are consistent with an infalling population that has entered the central $0.5 r_{200,3 \mathrm{D}}$ cluster region within the last $\sim 1$ Gyr. In the eight of nine cluster HDSGs with central star formation, the extent of star formation is consistent with that expected of outside-in quenching by ram pressure stripping. Our results indicate that the cluster HDSGs are currently being quenched by ram pressure stripping on their first passage through the cluster.
\end{abstract}

Key words: galaxies: clusters: general - galaxies: evolution - galaxies: star formation

\section{Introduction}

One of the key problems in modern astrophysics is understanding how galaxies evolve, with the process likely governed by both internal and external influences that manifest as well-defined correlations between galaxy properties, stellar mass, and external environment. The sense of the correlations is clear: the fraction of galaxies that are bulge dominated and devoid of star formation increases with stellar mass and local density, while the fraction of disk-dominated star-forming (SF) galaxies (SFGs) increases toward lower stellar mass and lower local density (Dressler 1980; Lewis et al. 2002; Kauffmann et al. 2003b). The relative importance of internal and external

\footnotetext{
${ }^{18}$ Hubble Fellow.
}

influences that act to stop the star formation in galaxies has been the subject of much study, with significant advances made possible by large surveys such as the $2^{\circ}$ Field Galaxy Redshift Survey (Colless et al. 2001) and Sloan Digital Sky Survey (SDSS; York et al. 2000). The large sample sizes provided by these surveys have helped to separate the effects of mass and environment, and indicate that the environment plays an important role in quenching star formation in galaxies (Balogh et al. 2004; Blanton \& Moustakas 2009; Peng et al. 2010). However, the dominant physical mechanisms responsible for the environment-driven quenching is still the subject of intense debate.

The impact of environmental quenching should reveal itself most prominently in the relatively hostile environments that exist in clusters of galaxies. There are a number of physical 
mechanisms that may act in clusters to both trigger and truncate star formation in infalling galaxies (see Boselli \& Gavazzi 2006, for a review). The processes can be divided into two categories: (i) interactions between the gas bound to the galaxy and the hot $\left(10^{7}-10^{8} \mathrm{~K}\right)$, rarefied $\left(10^{-3}\right.$ particles $\left.\mathrm{cm}^{-3}\right)$ intracluster medium (ICM), and (ii) gravitational interactions between either the galaxy and the cluster's gravitational potential, or interactions with other cluster galaxies.

Interactions with the ICM, such as ram pressure and viscous stripping (Gunn \& Gott 1972; Nulsen 1982), can easily remove the hot gas halo reservoir, thereby leading to a gradual decline in star formation (strangulation; Larson et al. 1980; Bekki et al. 2002; Bekki 2009). Strong ram pressure stripping can also remove the cold disk gas that fuels star formation, leading to quenching of star formation on short timescales (Roediger \& Brüggen 2006; Bekki 2014; Boselli et al. 2014a; Lee et al. 2017). These hydrodynamical interactions are able to affect galaxy star formation with little impact on the structure of the old stellar population.

The effect of gravitational interactions, through either tides due to the cluster potential, other galaxies, or the combined effect (harassment; Moore et al. 1996), can disrupt both the distribution of old stars and the gas in a cluster galaxy. This disruption may lead to transformations in the morphological, kinematical, SF, and active galactic nucleus (AGN) properties of cluster galaxies (Byrd \& Valtonen 1990; Bekki 1999). Galaxy-galaxy mergers are less frequent in the cores of clusters because of the high relative velocities of the galaxies (Ghigna et al. 1998). However, both simulations (McGee et al. 2009) and observations (Haines et al. 2018) indicate that 40\%-50\% of galaxies observed in massive clusters are accreted through smaller, group-scale halos (although the exact fraction depends on both halo and galaxy mass; De Lucia et al. 2012). Within these less-massive halos, the relative velocities between galaxies are lower, and preprocessing due to mergers and slower tidal interactions may be important (Cortese et al. 2006; Bianconi et al. 2018). Clearly, there are many mechanisms by which the cluster environment can act to quench the star formation in a galaxy. The outstanding challenge is to disentangle the impacts each of these mechanisms has, individually, on the star formation of recently accreted galaxies, and to understand the timescales required for them to transition from SF into quiescence.

Along these lines, it has been shown that the star formation rate (SFR) of SFGs within the central $R_{200}$ of clusters is systematically lower than that of SFGs in the field (e.g., Gavazzi et al. 2002, 2006; Koopmann \& Kenney 2004a; Haines et al. 2013). Furthermore, the mean SFR of SFGs is seen to decline steadily from the outskirts to the centers of clusters (von der Linden et al. 2010; Paccagnella et al. 2016; Barsanti et al. 2018). The slow decline in SFR with radius, coupled with kinematical evidence revealing that SFGs are consistent with being drawn from an infalling population (Colless \& Dunn 1996; Haines et al. 2015), indicate that the cluster environment acts to quench the star formation of infalling galaxies on timescales longer than a few billion years. Similar conclusions were reached by Taranu et al. (2014), who found that in order to match the reddening of disk colors toward the cluster center observed by Hudson et al. (2010), quenching must occur on relatively long $\sim 3$ Gyr timescales after infall. These relatively long timescales favor mild processes such as strangulation as being responsible for quenching.

However, other studies have found that the properties of cluster SFGs do not differ markedly from those of their field counterparts (Balogh et al. 2004; Muzzin et al. 2012; Wetzel et al. 2012). This finding has led to the proposal of the "delayed-then-rapid" quenching scenario by Wetzel et al. (2013), where SFGs are unaffected by the environment for several gigayears after becoming a satellite of a massive halo, before rapidly quenching on timescales shorter than $\sim 1 \mathrm{Gyr}$. The rapid phase of quenching is required to explain the strong bimodality observed in the SFR of cluster galaxies; there is a dearth of "green valley" galaxies with intermediate SFRs that are expected to exist if quenching acts on long timescales. A similar conclusion was reached by Oman \& Hudson (2016), who found that all galaxies become quenched on first infall, shortly after first pericentric passage.

Studies involving large, statistically significant samples of cluster galaxies allow constraints to be placed on overall quenching timescales. While these constraints help to understand which quenching mechanisms may be important, they do not allow for a detailed investigation of the processes at play. A complementary approach in this regard is to identify galaxies that show evidence for environmental perturbation, or transition galaxies that show evidence for very recent quenching, and target them with more detailed investigations. This approach has been successfully applied to galaxies in the nearby Virgo cluster where Chung et al. (2007, 2009a) have characterized the H I morphology of a sample of spiral galaxies. They find that galaxies within $0.5 \mathrm{Mpc}$ of M87 have much smaller H I disks when compared with the stellar disks, while many galaxies at larger cluster-centric radii show one-sided tails that point away from the cluster core, concluding that these galaxies are being influenced by ram pressure stripping on first infall.

Using $\mathrm{H} \alpha$ imaging, Koopmann \& Kenney (2004b) found that the distribution of star formation is truncated with respect to the stellar disk in the majority of the Virgo spirals that they studied. Very few SFGs in Virgo show an overall disk-wide reduction in SFR, indicating that ram pressure stripping is more important than strangulation for Virgo spirals. Crowl \& Kenney (2008) used integral-field spectroscopy (IFS) to follow up on a sample of 10 truncated spirals selected from the Koopmann \& Kenney (2004b) sample. They found that in all cases, the stellar populations in the regions just outside the radius of truncation were young $(<500 \mathrm{Myr})$, indicating that the cessation of star formation following the stripping of gas occurs on short timescales. While these observations point to the importance of ram pressure stripping in quenching star formation in Virgo, it must be emphasized that the centers of the truncated spirals in Virgo generally show normal SFRs. Crucially, transition galaxies analogous to those observed in Virgo but that exist in higher redshift clusters would be characterized as normal SFGs in single-fiber surveys. Therefore, key questions remain as to whether the Virgo-specific results are representative of the general cluster population.

Poststarburst galaxies are among the best candidates for galaxies that are in the process of transitioning from SF to quiescent systems. They were first identified in the spectroscopic surveys of intermediate redshift clusters as galaxies that exhibit strong Balmer absorption and an absence of emission lines excited by ongoing star formation (Dressler \& Gunn 1983; 
Couch \& Sharples 1987). Spectrophotometric modeling indicates that very strong Balmer absorption (i.e., $\mathrm{EW}(\mathrm{H} \delta)<$ $-5 \AA$ ) can only occur by the rapid truncation of a starburst within the last $\sim 1$ Gyr (Couch \& Sharples 1987). The weaker Balmer absorption seen in $\mathrm{H} \delta$-strong (HDS) galaxies (HDSGs) $(-5 \AA<\mathrm{EW}(\mathrm{H} \delta)<-3 \AA)$ is likely associated with recent truncation of normal star formation (also referred to as postSFGs; Couch \& Sharples 1987; Poggianti et al. 1999). Their transitioning state has made HDSGs attractive targets for attempting to identify the mechanism/s associated with the rapid quenching of star formation.

Comparisons between the environments and properties of HDSGs indicate that field HDSGs are likely the result of galaxy-galaxy mergers (Zabludoff et al. 1996; Blake et al. 2004; Yang et al. 2008; Pracy et al. 2009), while ICM-related stripping mechanisms are thought to be responsible for the quenching of cluster HDSGs (Poggianti et al. 1999; Tran et al. 2003; Muzzin et al. 2014; Paccagnella et al. 2017). Most previous studies rely on single-fiber or single-slit spectroscopy to identify the HDS spectral signature. Therefore, in order for HDSGs to be identified, either the entire galaxy must be completely quenched of star formation, or the aperture through which the spectrum is measured must be coincident with a post-SF region (e.g., as seen in Pracy et al. 2014). Thus, galaxies that are currently being transformed in an outside-in manner, such as those seen in Virgo by Crowl \& Kenney (2008), will not be identified in such surveys owing to aperture effects. Further, the unresolved nature of the spectra mean that contributions from HDS and SF regions cannot be disentangled.

Because many environment-related mechanisms modulate star formation in a spatially nonuniform way, the spatially resolved information provided by IFS makes it a powerful tool for understanding environment-related quenching. To date, the predominantly monolithic IFS instruments have meant that the focus of these observations has been on following up galaxies that are preselected because they show evidence for recent quenching or for being perturbed by the environment (Pracy et al. 2005; Merluzzi et al. 2013, 2016; Fumagalli et al. 2014; Fossati et al. 2016, 2018; Bellhouse et al. 2017; Fritz et al. 2017; Gullieuszik et al. 2017; Poggianti et al. 2017). The advent of multi-IFS instruments such as the Sydney-AAO Multi-Object IFS (SAMI; Bland-Hawthorn et al. 2011; Croom et al. 2012; Bryant et al. 2014) has opened up a new era for galaxy surveys where resolved spectroscopy can be collected for large, unbiased samples of galaxies.

Our aim in this paper is to use data from the SAMI Galaxy Survey (hereafter SAMI-GS; Bryant et al. 2015) to identify galaxies that exhibit evidence for recent quenching in their spatially resolved spectroscopy, and to understand how the environment may be acting to quench the star formation in these galaxies. We build upon previous studies that used the SAMI-GS to investigate quenching and environment (e.g., Schaefer et al. 2017, 2019; Medling et al. 2018) by both expanding the sample size and focusing on the cluster regions. Furthermore, we use the resolved spectroscopy to characterize both the ongoing SF distribution and to identify HDS regions associated with recent quenching. Critically, the SAMI-GS probes a broad range in environmental densities. The main portion of the survey targeted the equatorial GAMA regions (Galaxy and Mass Assembly; Driver et al. 2011) that contain low- to intermediate-density environments, and added eight massive clusters (Owers et al. 2017), allowing us to extend the work of Crowl \& Kenney (2008) to a larger, more representative sample of clusters. Toward that aim, we have used resolved spectroscopic classification maps from a sample of 1220 SAMI galaxies with $\log \left(M_{*} / M_{\odot}\right)>10$ and spanning all environments to identify 26 galaxies where more than $10 \%$ of their classifiable spaxels ${ }^{19}$ exhibit strong Balmer absorption, indicating recent quenching in those regions. We investigate the properties of these galaxies, focusing mainly on the HDSGs found in the cluster regions.

The outline of this paper is as follows: in Section 2, we describe the SAMI-GS and ancillary data used in this paper, as well as the sample selection. In Section 3, we describe our emission and absorption line measurements, Section 4 describes the spectroscopic classification maps, and Section 5 describes our classification of galaxies as passive galaxies (PASGs), SFGs, or HDSGs. In Section 6, we investigate the demographics of the HDSGs, paying particular attention to the environments of the cluster HDSGs, which we find are significantly different from those found in the GAMA regions, as well as being spatially and kinematically distinct from the cluster PASGs and SFGs. In Section 7, we interpret our results, showing that the cluster HDSGs are consistent with a recently accreted population of SFGs that are being quenched from the outside-in because of the effects of ram pressure stripping. Finally, in Section 8, we summarize our results and present our conclusions. Throughout this paper, we assume a standard $\Lambda \mathrm{CDM}$ cosmology, with $\Omega_{m}=0.3, \Omega_{\Lambda}=0.7$, and a HubbleLemaitre constant $H_{0}=70 \mathrm{~km} \mathrm{~s}^{-1} \mathrm{Mpc}^{-1}$.

\section{Data and Sample Selection}

In this section, we describe the SAMI-GS data, the ancillary data used, and the selection of the SAMI-GS galaxies used in this paper.

\subsection{The SAMI Galaxy Survey}

The SAMI-GS was conducted with the Sydney-AAO Multiobject Integral-field spectrograph (SAMI; Croom et al. 2012), which was mounted at the prime focus of the $3.9 \mathrm{~m}$ AngloAustralian Telescope and provided a $1^{\circ}$ diameter field of view (FOV). SAMI uses 13 fused fiber bundles (Hexabundles; Bland-Hawthorn et al. 2011; Bryant et al. 2014) with a high (75\%) fill factor. Each bundle contains 61 fibers of 1 !" 6 diameter, resulting in each integral field unit (IFU) having a diameter of $15^{\prime \prime}$. The IFUs, as well as 26 sky fibers, are plugged into predrilled plates using magnetic connectors. SAMI fibers are fed to the double-beam AAOmega spectrograph (Saunders et al. 2004; Sharp et al. 2006). AAOmega allows a range of different resolutions and wavelength ranges. For the SAMI-GS, we used the $570 \mathrm{~V}$ grating at 3700-5700 $\AA$ giving a central resolution of $R=1812$ in the blue $\operatorname{arm}\left(\sigma=70 \mathrm{~km} \mathrm{~s}^{-1}\right.$; FWHM $=2.65 \AA$ ), and the $1000 R$ grating from 6250 to $7350 \AA$ giving a central resolution of $R=4263$ in the red $\operatorname{arm}\left(\sigma=30 \mathrm{~km} \mathrm{~s}^{-1}, \mathrm{FWHM}=1.61 \AA\right.$ A ; van de Sande et al. 2017).

The SAMI-GS involved the observation of 3071 galaxies between 2013 and 2018 in the stellar mass range $\log \left(M_{*} / M_{\odot}\right)=8-12$ and with redshift $0.004<z \leqslant 0.115$. The SAMI-GS galaxies are primarily drawn from the equatorial G09, G12, and G15 GAMA I regions (2153 observed galaxies;

\footnotetext{
${ }^{19}$ Here and throughout this paper, the term spaxel refers to the spatial element of the IFS data cube.
} 
Driver et al. 2011; Liske et al. 2015), and also include galaxies selected from regions containing eight massive clusters with virial masses in the range $\log \left(M_{200} / M_{\odot}\right)=14.25-15.19(918$ observed galaxies; Owers et al. 2017). The input catalogs for the GAMA and cluster regions targeted during the SAMI-GS are described in detail in Bryant et al. (2015) and Owers et al. (2017). Briefly, the primary SAMI-GS targets in the GAMA regions are selected from a series of redshift bins with an increasing stellar mass limit in higher redshift bins. In the cluster regions, primary targets are selected using similar redshift-dependent stellar mass cuts, although a lower limit is set at $\log \left(M_{*} / M_{\odot}\right)=9.5$. Furthermore, primary targets in the cluster regions are constrained to have projected cluster-centric distance $R<R_{200}$, and peculiar velocity $\left|v_{\text {pec }}\right|<3.5 \sigma_{200}$ with respect to the cluster redshift, where $\sigma_{200}$ is the cluster velocity dispersion measured within $R_{200}$. For both the GAMA and cluster regions, a number of secondary targets with relaxed selection criteria are also included in the observations. The secondary objects are excluded from the analysis presented in this paper.

The observing procedure is detailed in Green et al. (2018). Briefly, each observed field involves a series of seven-dither pointings designed to provide both complete coverage over the $15^{\prime \prime}$ diameter FOV for each hexabundle, and to reduce the impact on image quality of the 1 ". 6 diameter fiber size, which undersamples the seeing point-spread function (PSF). Each dither pointing has a duration of $1800 \mathrm{~s}$, for a total $12,600 \mathrm{~s}$ exposure, and the seven-dither series is bookended by flat field and arc frames. When possible, twilight flats are observed for the purpose of fiber tracing, throughput, and flat-fielding. In cases where twilight flats could not be observed, dome flats are used in their place. Each plate observes 12 galaxies and one calibration star that is used for telluric and flux calibration. The data were reduced using the SAMI PYTHON package (Allen et al. 2014), which incorporates the 2DFDR package (AAO software team 2015). The reduced and calibrated data cubes are sampled on a regular spatial grid with $0 . " 5 \times 0$." 5 spaxels, and the spectra have pixel scales of 1.03 and $0.56 \AA$ for the blueand red-arm spectra, respectively. The full end-to-end description of reducing the data from raw frames to fully calibrated data cubes is described elsewhere (Allen et al. 2015; Sharp et al. 2015; Green et al. 2018; Scott et al. 2018).

\subsection{Ancillary Data}

We make use of several existing data products during our analysis. For the GAMA portion of the survey, the stellar masses, $M_{*}$, are determined using the approximation of Taylor et al. (2011) as outlined in Bryant et al. (2015), and use the aperture-matched $g$ - and $i$-band colors determined by Hill et al. (2011). Structural parameters (effective radius, $r_{e}$, Sérsic index, $n_{\text {ser }}$, ellipticity, and position angle (PA)) for the GAMA regions are drawn from the Sérsic profile fitting of SDSS $r$-band images as described in Kelvin et al. (2012). In the cluster regions, the same stellar mass proxy described in Bryant et al. (2015) is used to determine $M_{*}$, along with aperture-matched $g$ - and $i$-band magnitudes as described in Owers et al. (2017). We also make use of the cluster masses $\left(M_{200}\right)$, velocity dispersions $\left(\sigma_{200}\right)$, cluster redshifts $\left(z_{\text {clus }}\right)$, galaxy peculiar velocities $\left(v_{\text {pec }}\right)$, and overdensity radii $\left(R_{200}\right)$ published in Owers et al. (2017).

\subsubsection{Sérsic Fits for Cluster Galaxies}

Structural parameters for the cluster galaxies were determined from Sérsic profile fitting using the PROFIT ${ }^{20}$ code (Robotham et al. 2017). The fitting was performed on $r$-band images taken from the SDSS (DR9; Ahn et al. 2012) and VLT Survey Telescope (VST)/ATLAS (Shanks et al. 2015) surveys. The VST/ATLAS data were reprocessed as described in Owers et al. (2017) using the ASTRO-WISE pipeline as described in McFarland et al. (2013) and de Jong et al. (2015). At the position of each SAMI target in the cluster input catalogs, a $400^{\prime \prime} \times 400^{\prime \prime}$ cutout image was generated in each of the available bands. Pointlike objects were selected on the basis of their position in the size-surface-brightness plane, and nonsaturated point sources with magnitude $16<r<20$ were fitted with Moffat profiles using PROFIT. The median of the best-fit parameter values is used to generate a PSF specific to each $r$-band cutout, and this PSF is used for convolution during the Sérsic profile fitting.

Prior to fitting, we perform local sky subtraction on each cutout after aggressively masking detected sources. We use the PROFOUND $^{21}$ software package (Robotham et al. 2018) to generate a detection image from an inverse-variance-weighted stack of the griz-band images (or gri-band in the case of VST/ ATLAS, where the $z$-band was not available). We then run PROFOUND on the detection image to detect and characterize the shapes of sources in the field. The shape parameters derived with PROFOUND (i.e., position, PA, and axial ratio) were used to generate a mask around each detected object as follows. We use the PROFIT software to produce a Sérsic model for each object using the PROFOUND-derived magnitude and shape parameters, and assuming Sérsic index $n_{\text {ser }}=4$, which is typical of the early-type galaxies found in the clusters. We use the model to mask all pixels within a constant surface brightness $\mu=30 \mathrm{mag} \operatorname{arcsec}^{-2}$ for each object. This aggressive masking ensures that the remaining pixels are not contaminated by the faint outer wings of galaxies. We then define a $10^{\prime \prime} \times 10^{\prime \prime}$ grid and determine the local sky at each gridpoint using a box with an adaptive size that is grown until the box contains 10,000 unmasked sky pixels. The sky and sky noise are determined from the distribution of values in the adaptive box. Masked object regions are then interpolated using inverse-distance-weighted means, and the gridded sky distribution is interpolated back to the full-resolution grid using bicubic interpolation. This final sky distribution is subtracted from the $r$-band image prior to fitting.

During Sérsic fitting, any galaxy for which R100 (the elliptical semimajor axis that contains $100 \%$ of the flux as defined by PROFOUND) overlaps with that of the primary galaxy of interest, and that also has an isophotal area greater than $5 \%$ of the primary galaxy's isophotal area, is simultaneously fitted along with the primary galaxy. Stars and objects that do not meet these criteria are masked using the segmentation map derived with PROFOUND. Initial input estimates for the Sérsic profile parameters are derived from the PROFOUND outputs and further optimized via the $R$ optim function using the "L-BFGS-B" algorithm. The final parameters are determined using the LaplaceDemon package, where we run 10,000 Markov chain Monte Carlo (MC) iterations using the Componentwise Hit-And-Run method.

\footnotetext{
${ }^{20}$ https://github.com/ICRAR/ProFit

21 https://github.com/asgr/ProFound
} 

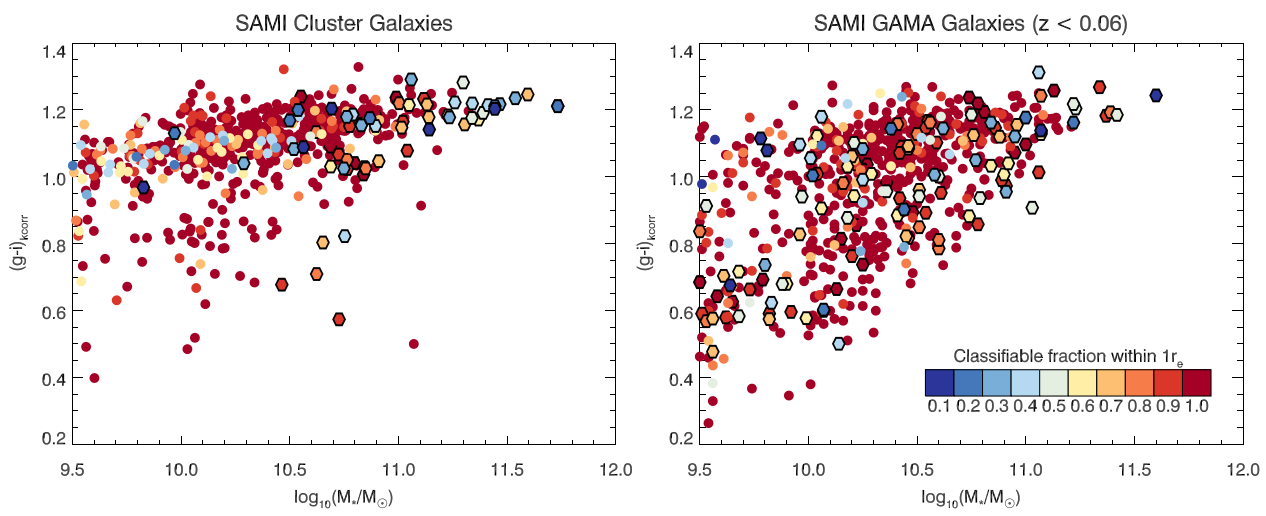

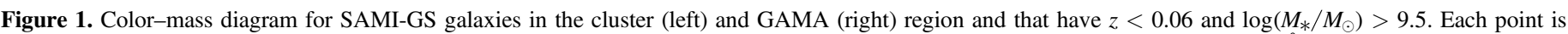

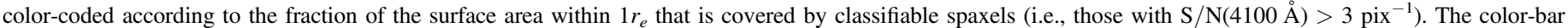

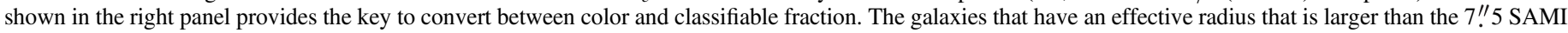
hexabundle radius are plotted as hexagons.

We checked our fit parameters for both internal and external consistency. Internal checks were performed on a subset of 143 SAMI galaxies in the cluster A85 that have both VST/ATLAS and SDSS imaging. We found that the distribution of the relative difference between the SDSS and VST/ATLAS PA and ellipticity measurements were smaller than $1 \%$, with dispersion $3.3 \%$ and $1 \%$, respectively, indicating very good agreement between the two imaging surveys for these two parameters. However, we found a systematic offset in $r_{e}$ and $n_{\text {ser }}$ of the order $3.5 \%-4 \%$, with the SDSS values being larger on average than those derived using the VST/ATLAS data. We also found a larger scatter of $6 \%$ and $10 \%$ for the relative differences in the $r_{e}$ and $n_{\mathrm{ser}}$ measurements. Dividing the 143 galaxies into those galaxies with $n_{\text {ser }}>2$ and $n_{\text {ser }}<2$ (i.e., disk- and bulge-dominated galaxies, respectively), we found that the systematic offsets in the relative difference for both $r_{e}$ and $n_{\text {ser }}$ are due to differences in the bulge-dominated sample. These systematic offsets are likely due to the oversubtraction of the sky around these larger objects in the VST/ATLAS data, which leads to a steepening of the outer profile and, therefore, smaller $r_{e}$ and $n_{\text {ser }}$ values when compared with those derived from the SDSS imaging. These systematic offsets are small and do not affect our conclusions. External checks are performed for the SDSS fits by comparing our results for a sample of 557 galaxies matched to the single Sérsic fits from Meert et al. (2015). We found good agreement, with the relative differences between $r_{e}, n_{\text {ser }}$, axial ratio, and PA differing by less than $2 \%$, and scatter smaller than $10 \%$.

\subsection{Sample Selection}

The sample of galaxies used in this paper is selected from the 2526 SAMI-GS galaxies observed prior to 2017 September (internal team release version V0.10.1; 894 cluster and 1632 GAMA galaxies). In the cluster regions, we consider only the 714 primary target galaxies that are allocated as cluster members in Owers et al. (2017). In order to better match the stellar mass and redshift distributions of the cluster sample, the GAMA sample includes only the 791 primary targets that have $z<0.06$ and $\log \left(M_{*} / M_{\odot}\right)>9.5$. For galaxies with multiple observations, we use the data from the observation taken in the best seeing conditions. The completeness of the GAMA portion of the sample is lower than that of the cluster sample (61\% c.f. $87 \%$, respectively), although there is no apparent stellar mass bias in the completeness.
In addition to the selection described above, in our final sample we include only the 579 cluster and 649 GAMA galaxies with $\log \left(M_{*} / M_{\odot}\right) \geqslant 10$. There are two reasons for this additional selection criterion: first, for the clusters in the survey with $z>0.045$, galaxies with $\log \left(M_{*} / M_{\odot}\right)<10$ were not observed as primary targets; below this cutoff only those galaxies in the blue cloud were included as secondary targets (Owers et al. 2017). Removing objects with $\log \left(M_{*} / M_{\odot}\right)<10$ therefore allows for a homogeneous selection both within the cluster sample and across the GAMA and cluster samples. Second, we wish to perform resolved spectroscopic classification based on both absorption and emission line measurements (outlined below in Section 4). The absorption line classification requires signal-to-noise ratio $(\mathrm{S} / \mathrm{N})(4100 \AA)>3 \mathrm{pix}^{-1}$ (see Sections 3.3 and 4.2 ) so that we can reliably classify spaxels on the basis of the strength of the Balmer line absorption. To perform the galaxy classifications outlined in Section 5.2, it is desirable that more than 100 spaxels meet this $\mathrm{S} / \mathrm{N}$ criteria. This 100 spaxel area corresponds to that contained within a circular region with diameter $\sim 5$ ! 6 , which is substantially larger than the mean seeing FWHM $=2$ ".06 (Scott et al. 2018). When comparing the GAMA and cluster samples, we found that $57 \%(77 / 135)$ of cluster galaxies with $9.5 \leqslant$ $\log \left(M_{*} / M_{\odot}\right)<10$ had fewer than 100 spaxels that met the $\mathrm{S} / \mathrm{N}$ criterion, whereas this was the case for only $14 \%(20 / 142)$ of low-mass galaxies in the GAMA regions. For $\log \left(M_{*} / M_{\odot}\right) \geqslant 10, \sim 86 \%$ and $90 \%$ of SAMI galaxies in the cluster and GAMA regions, respectively, have more than 100 $\mathrm{S} / \mathrm{N}(4100 \AA)>3$ pix $^{-1}$ spaxels. Therefore, selecting galaxies with $\log \left(M_{*} / M_{\odot}\right) \geqslant 10$ allows for relatively unbiased comparisons to be performed between the two samples.

To investigate potential systematic biases in the spatial coverage of our spectral classification maps, we present Figure 1 , which shows the $(g-i)_{\mathrm{kcorr}}$ versus $\log \left(M_{*} / M_{\odot}\right)$ color-mass plane for the galaxies in the cluster and GAMA regions (left and right panels, respectively). In Figure 1, $(g-i)_{\mathrm{kcorr}}$ is the $k$-corrected color where the $k$-correction has been determined using the CALC_KCOR $\operatorname{code}^{22}$ from Chilingarian et al. (2010). Each point in Figure 1 is color-coded according to the fraction of the surface area contained within one effective radius for which there are spaxels with $\mathrm{S} / \mathrm{N}(4100 \AA)>3 \mathrm{pix}^{-1}, f_{\text {class }, r_{e}}$. A significant portion of the

\footnotetext{
${ }^{22}$ http://kcor.sai.msu.ru/getthecode/
} 
red-sequence cluster galaxies with $9.5 \leqslant \log \left(M_{*} / M_{\odot}\right)<10$ have a relatively low $f_{\text {class, } r_{e}}$ when compared with blue-cloud galaxies within the same $\log \left(M_{*} / M_{\odot}\right)$ range. This systematic bias further justifies our decision to include only $\log \left(M_{*} / M_{\odot}\right)>10$ galaxies in our sample. Within our sample of $\log \left(M_{*} / M_{\odot}\right) \geqslant 10$ galaxies, $86 \%(87 \%)$ of cluster (GAMA) galaxies have $f_{\text {class, } r_{e}} \geqslant 0.7$. Of the galaxies that have $\log \left(M_{*} / M_{\odot}\right) \geqslant 10$ and $f_{\text {class, } r_{e}}<0.7$, a significant fraction $(48 \%$ and $65 \%$ in the cluster and GAMA regions, respectively) have an effective radius that is larger than the SAMI hexabundle size (i.e., they have $r_{e}>7$ !! 5); these galaxies are plotted as hexagons in Figure 1. This effect is most prevalent at high masses $\left(\log \left(M_{*} / M_{\odot}\right)>11.2\right)$, where almost all galaxies are affected. Aside from the systematic bias at large stellar masses, which equally affects both the cluster and GAMA samples, there do not appear to be any prominent biases in the $f_{\text {class, } r_{e}}$ across the color-mass plane.

\section{Line Strength Measurements}

The spectral classification scheme outlined in Section 4 requires measurements of emission and absorption line strengths. In this section, we describe the procedure for defining the stellar continuum and for measuring emission and absorption line fluxes and equivalent widths.

\subsection{Stellar Continuum Definition}

Accurate emission line flux measurements require that the stellar continuum be modeled and subtracted. This is particularly important for the Balmer lines, which can be significantly affected by underlying stellar absorption. The fidelity of the stellar continuum fit depends strongly on the $\mathrm{S} / \mathrm{N}$ in the continuum of the spectrum. For accurate stellar continuum modeling, it is common to spatially bin spectra to reach a minimum $\mathrm{S} / \mathrm{N}$ in the continuum (e.g., Cappellari \& Copin 2003). However, often the binning scheme used for continuum modeling is not suitable for emission lines, which can have good $\mathrm{S} / \mathrm{N}$ in the unbinned data. For this reason, we employ a hybrid approach that uses a combination of binned and full spatial resolution data and is outlined below.

We use the penalized pixel fitting software (pPXF; Cappellari \& Emsellem 2004; Cappellari 2017), in combination with 73 Stellar Population Synthesis (SPS) templates drawn from the MILES (Vazdekis et al. 2010) and González Delgado et al. (2005) libraries, to fit the underlying stellar continuum for each spaxel. From the MILES SPS library, we select a subset of templates that contains four metallicities $([\mathrm{M} / \mathrm{H}]=-0.71$, $-0.40,0.00,0.22)$ and 13 logarithmically spaced ages ranging from 0.0063 to 15.85 Gyr. Following Cid Fernandes et al. (2013), we also include the subset of González Delgado et al. (2005) SPS templates with metallicities $[\mathrm{M} / \mathrm{H}]=-0.71$, $-0.40,0.00$ and ages $0.001-0.025 \mathrm{Gyr}$, which extends the MILES coverage to younger ages. The continuum for each spaxel is determined using the following multistep process outlined below, and also in Figure 2.

\subsubsection{Refining Template Library Using Voronoi Binned Data}

We follow a procedure similar to that outlined in van de Sande et al. (2017), where we use the higher S/N spatially binned data to select a subset of the 73 SPS templates to use in fitting the lower $\mathrm{S} / \mathrm{N}$ single-spaxel data. This preselection of SPS templates helps to avoid overfitting of the noisier single-spaxel data. We use data that have been binned spatially to reach a $\mathrm{S} / \mathrm{N} \sim 10$ using Voronoi binning (Cappellari \& Copin 2003), where covariance between spaxels due to dithering has been accounted for when determining the variance of the combined spectrum (Allen et al. 2015; Sharp et al. 2015). Two examples of spectra resulting from the Voronoi binning are shown in panels (a) and (d) of Figure 2. Rather than refit the stellar kinematics, we use the existing twomoment (velocity and velocity dispersion) kinematic data that were described in van de Sande et al. (2017) to bring the spectra and templates to a common rest frame and dispersion. The galaxy spectra are corrected to the rest frame using the redshift $\left(1+z_{\text {tot }}\right)=\left(1+z_{\text {gal }}\right)\left(1+v_{\mathrm{pPXF}} / c\right)$, where $z_{\text {gal }}$ is the galaxy redshift, $v_{\mathrm{pPXF}}$ is the velocity with respect to $z_{\mathrm{gal}}$, and $c$ is the speed of light. We then convolve each SPS template using a Gaussian kernel with the wavelength-dependent width

$$
\sigma_{\text {tot }}^{2}=\left[\left(\frac{\lambda \sigma_{\mathrm{pPXF}}}{c}\right)^{2}+\left(\frac{\sigma_{\mathrm{inst}}}{1+z}\right)^{2}\right]-\sigma_{\text {MILES }}^{2}
$$

where $\sigma_{\mathrm{pPXF}}$ is the velocity dispersion (in $\mathrm{km} \mathrm{s}^{-1}$ ) of the spectrum determined by van de Sande et al. (2017), $\lambda$ is the wavelength of the pixel, $\sigma_{\text {inst }}=1.13 \AA(0.68 \AA)$ is the instrument resolution for the blue (red) arm of the spectrograph (van de Sande et al. 2017), and $\sigma_{\text {MILES }}=1.06 \AA$ is the resolution of the MILES templates (Falcón-Barroso et al. 2011). The SPS templates, the data, and the variance are rebinned onto a grid with constant velocity pixel size that is best matched to the blue-arm data (i.e., $\Delta v \sim 55 \mathrm{~km} \mathrm{~s}^{-1}$ ), thereby undersampling the red-arm data. We then use pPXF to determine the optimal combination of the MILES templates while fixing $v_{\mathrm{pPXF}}=0 \mathrm{~km} \mathrm{~s}^{-1}$ and $\sigma_{\mathrm{pPXF}}=0 \mathrm{~km} \mathrm{~s}^{-1}$. A 12th-order multiplicative polynomial is used to correct for any effects due to data reduction artifacts, and also the effects of dust extinction.

The above process is repeated twice. On the first iteration, the regions surrounding strong emission lines are masked. Following this first iteration, the error array associated with the spectrum is normalized by the ratio of the median absolute deviation of the residuals to the median of the error array. In the second iteration, the emission lines are not masked, and, in addition to the SPS templates, we include emission line templates for all Balmer lines from $\mathrm{H} \zeta(\lambda 3889)$ in the blue to $\mathrm{H} \alpha(\lambda 6563)$ in the red, as well as the strong forbidden lines [OII] $(\lambda \lambda 3726,3729),[\mathrm{O}$ III] $(\lambda \lambda 4959,5007),[\mathrm{OI}](\lambda \lambda 6300,6364)$, [N II] $(\lambda \lambda 6548,6583)$, and [S II] $(\lambda \lambda 6717,6731)$. We fit for the kinematics of the emission line templates, assuming the same kinematics for the Balmer and forbidden lines, and include the velocity, velocity dispersion, and the higher-order $h_{3}$ and $h_{4}$ components. Example emission line fits are overplotted on the stellar-continuum-subtracted, pure emission line spectra shown in the lower plots of panels (a) and (d) in Figure 2. We also use the CLEAN keyword in order to reject outliers and to ensure that the presence of weak emission lines does not impact the fit to the stellar continuum. Only those SPS templates with nonzero weights (shown in green in panels (a) and (d) of Figure 2) in this final iteration are used for the per-spaxel fitting outlined in Section 3.1.2. In addition, the emission line kinematics derived here serve as initial estimates for the kinematics of the per-spaxel emission line fitting. 

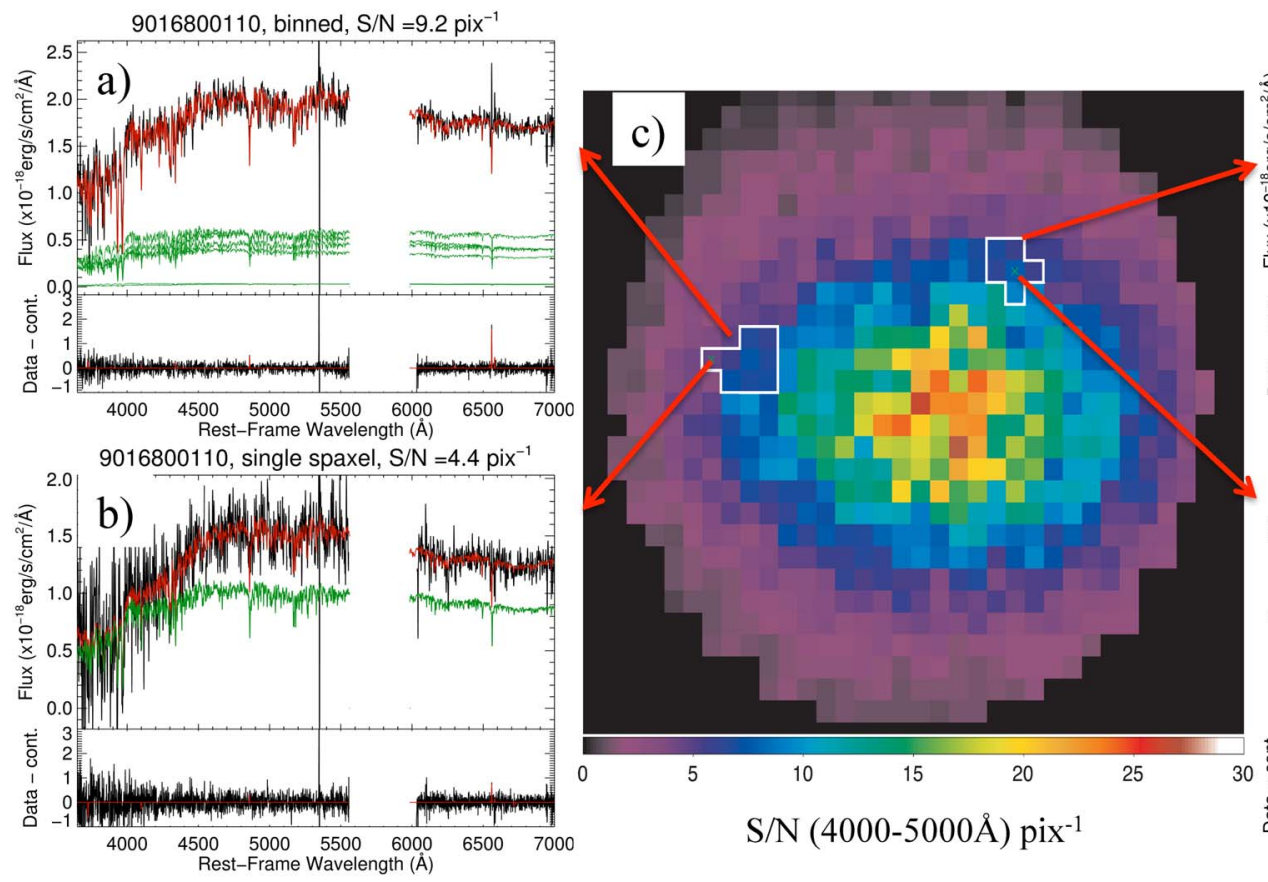

$\mathrm{S} / \mathrm{N}(4000-5000 \AA) \mathrm{pix}^{-1}$

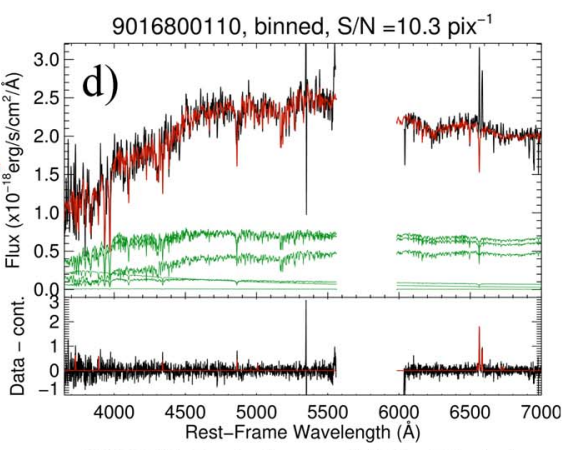

9016800110 , single spaxel, $\mathrm{S} / \mathrm{N}=7.7 \mathrm{pix}^{-1}$

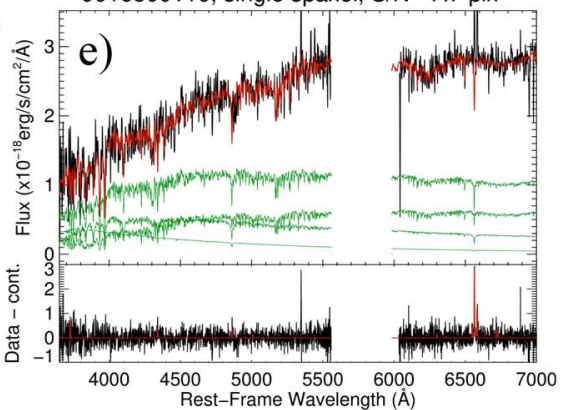

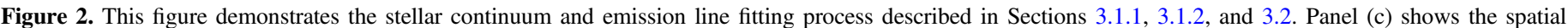

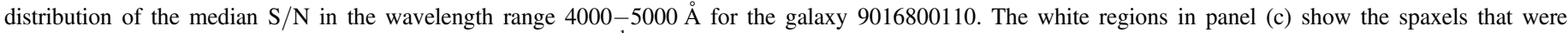

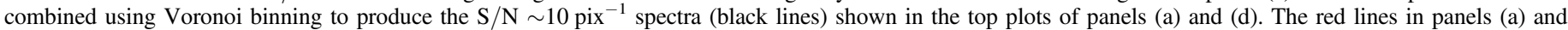

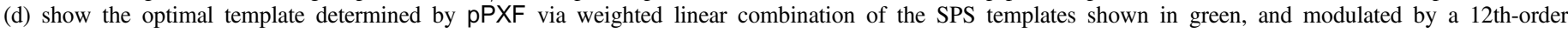

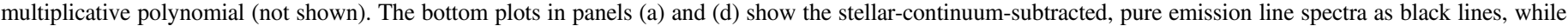

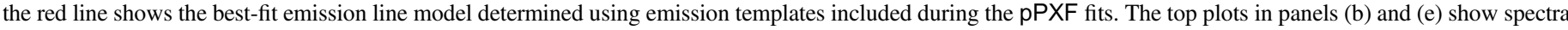

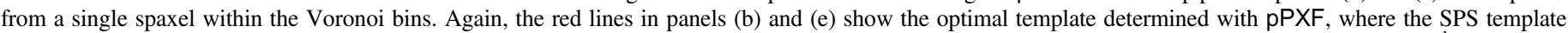

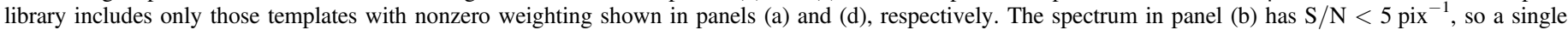

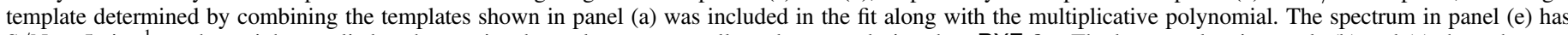

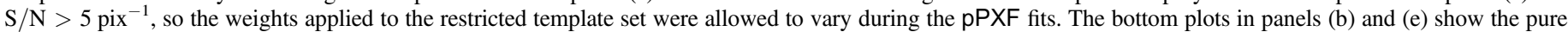
emission line spectra in black, while the red lines show the emission line models determined from the Gaussian fits described in Section 3.2 .

\subsubsection{Continuum Definition for Individual Spaxels}

Having refined the SPS library and determined initial estimates for the emission line kinematics, we now fit the spectrum of each spaxel contained in each of the Voronoi bins. The SPS templates, spectrum, and variance are rebinned to a pixel scale with constant velocity width that is tuned to best match the red arm (i.e., $\Delta v \sim 25 \mathrm{~km} \mathrm{~s}^{-1}$ ), thereby oversampling the blue-arm spectrum. During fitting, the forbidden and Balmer emission line species are assumed to have the same kinematics (velocity, dispersion, $h_{3}$, and $h_{4}$ ). The simultaneous fitting of the underlying stellar continuum and the emission lines allows for a better solution for the underlying stellar continuum to be found than if the emission lines were simply masked. This improvement is because important continuum regions surrounding emission lines can be included in the fit; in particular, the age-sensitive Balmer lines bluer than $\mathrm{H} \beta$ can now influence the fitted continuum.

For spaxels with $\mathrm{S} / \mathrm{N}>5$ in the blue arm, the stellar kinematics can be determined reliably (Fogarty et al. 2015). Therefore, those spaxels with $\mathrm{S} / \mathrm{N}>5$ have their stellar kinematics fixed to the per-spaxel value determined in van de Sande et al. (2017). For $\mathrm{S} / \mathrm{N}>5$ spaxels, we also allow pPXF to fit for the weights of the refined SPS template library, as well as including a 12th-order multiplicative polynomial that corrects for residual differences in the SPS templates and the data (see panel e in Figure 2). When the $\mathrm{S} / \mathrm{N}<5$, the stellar kinematics and SPS template weights are less well-constrained. For spaxels with $\mathrm{S} / \mathrm{N}<5$, we fix the stellar kinematics to the velocity and dispersion determined using the Voronoi binned spectrum by van de Sande et al. (2017). Furthermore, for $\mathrm{S} / \mathrm{N}<5$ spaxels, rather than fitting for the weights for the individual SPS templates, we use a single optimal template that is constructed using the weights determined in fitting the Voronoi binned spectrum. Thus, for $\mathrm{S} / \mathrm{N}<5$ spaxels, the only free parameters used in fitting the stellar continuum are a single weight for the optimal template, as well as the coefficients of the 12th-order multiplicative polynomial. This constrained fit allows for a more robust definition of the underlying stellar continuum even in lower $\mathrm{S} / \mathrm{N}$ regimes (see panel $\mathrm{b}$ in Figure 2).

\subsection{Emission Line Flux Measurements}

While the per-spaxel continuum fitting procedure described in Section 3.1 does produce emission line fluxes, the disparity between the pixel scales for the blue- and red-arm data means that the measurements are performed on heavily oversampled data in the blue. This oversampling may introduce inaccuracies in the measured fluxes, and the associated formal uncertainties. Instead, we use a Python implementation of mpfit ${ }^{23}$ (Markwardt 2009; Cappellari 2017) to fit the Gaussians to the emission lines after subtracting the best-fitting stellar continuum. The best-fit model for the stellar continuum determined in Section 3.1 .2 is redshifted to $z_{\text {tot }}$, interpolated onto the pixel

\footnotetext{
23 This code was converted from IDL to Python by Mark Rivers, Sergey Koposov, and Michele Cappellari.
} 
scale of the blue- and red-arm data (1.03 and $0.56 \AA$, respectively), and subtracted from the data, leaving a pure emission line spectrum as shown in the lower plots of panels (b) and (e) in Figure 2. The fitting of this emission line spectrum is outlined below.

The line shapes often exhibit non-Gaussian profiles, meaning that fluxes determined from fitting a single Gaussian component may substantially underestimate the total line flux (e.g., Ho et al. $2014,2016)$. To detect the presence of non-Gaussianity, we perform an initial fit to the $[\mathrm{N} \mathrm{III}](\lambda 6583), \mathrm{H} \alpha$, and $[\mathrm{S}$ II $](\lambda \lambda 6716,6731)$ emission lines, which fall in the highresolution portion of the SAMI spectra. First, we fit a single Gaussian profile with velocity and velocity dispersion fixed for the different line species. A second fit is then performed with the addition of the higher-order Gauss-Hermite $h_{3}$ and $h_{4}$ terms, which parameterize asymmetric and symmetric departures from a Gaussian shape, respectively (van der Marel \& Franx 1993). We use the change in the Bayesian Information Criterion, $\Delta \mathrm{BIC}=\mathrm{BIC}_{\mathrm{Gauss}}-\mathrm{BIC}_{\mathrm{Gauss}-H e r m i t e}$, to determine whether the extra two parameters describing departures from a Gaussian shape are justified. Here, BIC $=\chi^{2}+d(\ln (N)-\ln (2 \pi))$, where $N$ is the number of data points and $d$ is the number of free parameters in the fit. In the case that $\Delta \mathrm{BIC}<10$, a single Gaussian is deemed sufficient to describe the emission line shape. Where $\triangle \mathrm{BIC} \geqslant 10$, we perform a third iteration of fitting where we use two Gaussian components. For the first Gaussian component, the velocity and dispersion determined in the first step are used as input guesses. We use the derivatives of the best-fitting Gaussian-plus-Gauss-Hermite model to determine initial estimates for the second Gaussian component using Equations 2(a)-(4) in Lindner et al. (2015).

Having determined whether a one- or two-Gaussian profile best describes the emission line shape, we then include the emission lines in the blue arm of the spectrum. We fit the $\mathrm{H} \delta$ to $\mathrm{H} \alpha$ Balmer lines and the $[\mathrm{O} \mathrm{II}](\lambda \lambda 3726,3729),[\mathrm{O} \mathrm{III}](\lambda \lambda 4959,5007)$, [O I] $(\lambda \lambda 6300,6364),[\mathrm{N} \mathrm{II}](\lambda 6548,6583)$, and [S II] $(\lambda \lambda 6716$, $6731)$ doublets. The velocity and velocity dispersion of the Balmer and forbidden lines are fixed to the same value, with the different instrument resolution of the blue- and red-arm spectra appropriately accounted for. The velocity and velocity dispersion determined during the initial fits to the $[\mathrm{N} \mathrm{II}](\lambda 6583), \mathrm{H} \alpha$, and $[\mathrm{S} \mathrm{II}](\lambda \lambda 6716$, $6731)$ emission lines are used as initial inputs during the fitting to the full range of emission lines. The amplitudes of the [O III] $(\lambda \lambda 4959,5007),[\mathrm{O} \mathrm{I}](\lambda \lambda 6300,6364)$, and [N II] $(\lambda 6548$, $6583)$ doublets are fixed to their expected values of $0.347,0.333$, and 0.339 , respectively. Fluxes are determined for each line using the fitted amplitude and line dispersion. Uncertainties on the fluxes are determined by propagating the formal uncertainties on the amplitude and dispersion, and include covariance terms that can contribute significantly to the flux uncertainties obtained for the two-Gaussian cases. Throughout the remainder of this paper, we use the total emission line flux determined from the one- or twoGaussian profile that provides the best description of the emission line shape.

\subsection{Absorption Line Equivalent Widths}

Absorption line equivalent widths and uncertainties are determined using the direct summation method described in Cardiel et al. (1998). The bands used to define the line and continuum regions are shifted to the observed frame using the $z_{\text {tot }}$ determined in Section 3.1. Prior to measuring absorption line equivalent widths, the best-fitting emission line model is subtracted from the spectrum. This correction is performed only when the measured emission line kinematics are reliable - that is, the velocity and dispersion have not hit a boundary in the parameter space, nor is the amplitude of the emission line negative. When the emission line model is subtracted, the uncertainty on the absorption line equivalent widths include a contribution due to the uncertainty in the emission line flux measurement. We measure the age-sensitive Balmer absorption lines $\mathrm{H} \delta_{F}$ and $\mathrm{H} \beta$ using the definitions of Worthey \& Ottaviani (1997) and Worthey et al. (1994), respectively. The $\mathrm{H} \gamma_{F}$ equivalent width is determined using the line bandpass and red continuum sideband definitions of Worthey \& Ottaviani (1997) and the blue continuum sideband definition of Fisher et al. (1998). The shifting of the blue sideband helps to avoid contamination of the continuum measurement due to the $G$-band absorption at $4304 \AA$.

Many spaxels have $\mathrm{S} / \mathrm{N}(4100 \AA) \sim 3 \mathrm{pix}^{-1}$, and this is particularly prevalent in the outer parts of galaxies where environmental quenching may be more readily detected. The median uncertainty on $\mathrm{EW}\left(\mathrm{H} \delta_{F}\right)$ measurements for spectra with $\mathrm{S} / \mathrm{N}(4100 \AA)=3 \mathrm{pix}^{-1}$ is $\sigma_{\mathrm{EW}\left(\mathrm{H} \delta_{F}\right)} \sim 2 \AA$, meaning that we cannot reliably distinguish passive and HDS spectra; the median uncertainty drops below $\sim 1 \AA$ only when the $\mathrm{S} / \mathrm{N}$ $(4100 \AA) \sim 6 \mathrm{pix}^{-1}$. Rather than removing all $\mathrm{S} / \mathrm{N}(4100 \AA)$ $<6$ pix $^{-1}$ spaxels, or binning spatially to achieve a higher $\mathrm{S} / \mathrm{N}$ in the continuum (which is generally not optimal for emission line measurements), we follow a procedure similar to that of Blake et al. (2004) and use the correlation between the $\mathrm{EW}\left(\mathrm{H} \delta_{F}\right), \mathrm{EW}\left(\mathrm{H} \gamma_{F}\right)$, and $\mathrm{EW}(\mathrm{H} \beta)$ measurements to determine a higher $\mathrm{S} / \mathrm{N}$ proxy for $\mathrm{EW}\left(\mathrm{H} \delta_{F}\right)$.

Figure 3 shows the strong correlations between $\operatorname{EW}\left(\mathrm{H} \delta_{F}\right)$ and $\mathrm{EW}\left(\mathrm{H} \gamma_{F}\right)$ (left panel) and $\mathrm{EW}\left(\mathrm{H} \delta_{F}\right)$ and $\mathrm{EW}(\mathrm{H} \beta)$ (middle panel). The correlations are fitted with a linear relation using the HYPER-FIT ${ }^{24}$ package (Robotham \& Obreschkow 2015), which accounts for uncertainties in both the $x$ - and $y$-measurements. For the fitting, we only use measurements where $\mathrm{S} / \mathrm{N}(4100 \AA)>10, \mathrm{EW}(\mathrm{H} \alpha)<20 \AA$, and where $\mathrm{S} / \mathrm{N}$ $(\mathrm{EW})>2$ in absorption (where $\mathrm{S} / \mathrm{N}(\mathrm{EW})=|\mathrm{EW}| / \sigma_{\mathrm{EW}}$ ) for both EW measurements. The best-fit linear relations are shown in the lower right of both panels and also are plotted as black lines. We use the best-fit relations to produce a pseudo-EW $(\mathrm{H} \delta)$ from the $\operatorname{EW}\left(\mathrm{H} \gamma_{F}\right)$ and $\operatorname{EW}(\mathrm{H} \beta)$ measurements. Uncertainties on the pseudo-EW( $\mathrm{H} \delta)$ measurements are determined using standard error propagation and include measurement uncertainty, as well as contributions from the uncertainties on the fitted parameters, and intrinsic scatter in the relations as determined by HYPER-FIT.

For each spectrum, the $\mathrm{EW}\left(\mathrm{H} \delta_{F}\right)$ and pseudo- $\mathrm{EW}\left(\mathrm{H} \delta_{F}\right)$ measurements are combined using a weighted average of the three measurements. The weighting includes an inverse-variance term, as well as a term that deweights the contribution from outlier measurements. The final weighted average of the three measurements, hereafter $\mathrm{EW}\left(\overline{\mathrm{H}_{\delta \gamma \beta}}\right)$, is used for the classification described in Section 4.2. In the right panel of Figure 3 we show the comparison of the $\mathrm{EW}\left(\overline{\mathrm{H}_{\delta \gamma \beta}}\right)$ and $\mathrm{EW}\left(\mathrm{H} \delta_{F}\right)$ measurements for spectra with $\mathrm{S} / \mathrm{N}(4100 \AA)>6 \mathrm{pix}^{-1}, \mathrm{EW}(\mathrm{H} \alpha)<20 \AA$, and $\mathrm{S} / \mathrm{N}(\mathrm{EW})>2$ in absorption. There is a strong one-to-one correlation between the two measurements, which indicates that the method for determining $\mathrm{EW}\left(\overline{\mathrm{H}_{\delta \gamma \beta}}\right)$ does not introduce strong biases into the estimates of the $\mathrm{H} \delta$ strength. Moreover, for spectra

\footnotetext{
24 https://hyperfit.icrar.org
} 

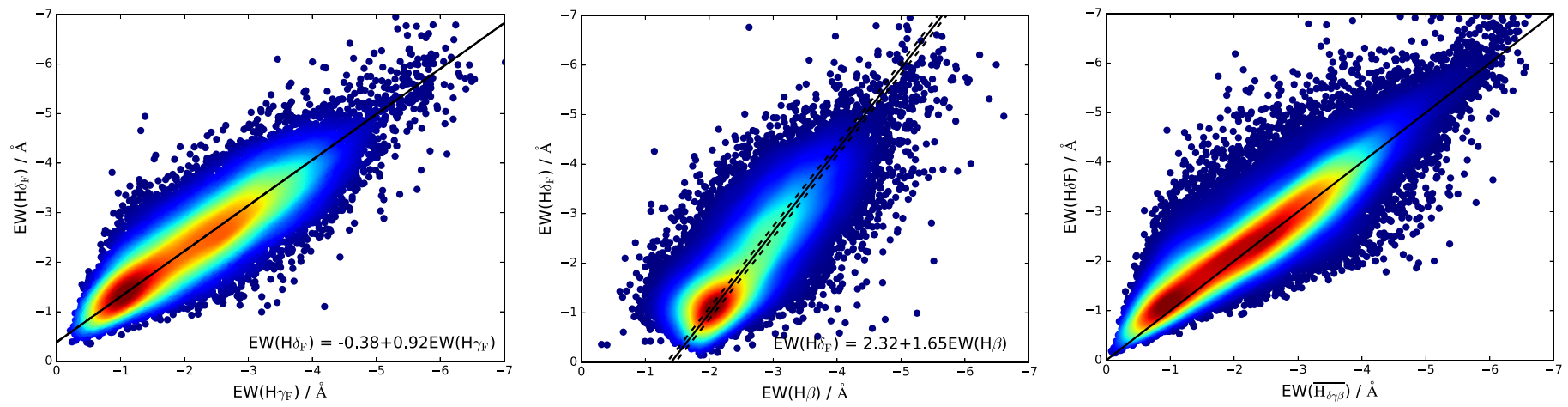

Figure 3. The left and middle panels show the $\operatorname{EW}\left(\mathrm{H} \delta_{F}\right)$ vs. $\operatorname{EW}\left(\mathrm{H} \gamma_{F}\right)$, and $\operatorname{EW}\left(\mathrm{H} \delta_{F}\right)$ vs. $\operatorname{EW}(\mathrm{H} \beta)$ plots, revealing the strong correlations that exist between the Balmer line absorption strengths. In these two panels, the black lines show the best-fit linear relations determined with the HYPER-FIT package, and the best-fit parameters are shown on the lower right. We use these best-fit linear relations to determine a weighted average $\mathrm{EW}\left(\overline{\mathrm{H}_{\delta \gamma}}\right)$, which is shown plotted against $\mathrm{EW}\left(\mathrm{H} \delta_{F}\right)$ in the right panel. In all three panels, the colorscale shows smoothed density distribution of points, which ranges from $1 \%$ of the peak density (dark blue) to $99 \%$ of the peak density (dark red) on a linear scale. The right panel only includes spectra with $\mathrm{S} / \mathrm{N}(4100 \AA)>6$ pix $^{-1}, \mathrm{EW}(\mathrm{H} \alpha)<20 \AA$, and where each EW is detected with $\mathrm{S} / \mathrm{N}>2$. The black line shows the one-to-one relation. For absorption stronger than $\mathrm{EW}=-1 \AA$, there is a strong one-to-one correlation between EW( $\left.\overline{\mathrm{H}_{\delta \gamma \beta}}\right)$ and $\mathrm{EW}\left(\mathrm{H} \delta_{F}\right)$, indicating that the weighted average used to determine $\mathrm{EW}\left(\overline{\mathrm{H}_{\delta \gamma \beta}}\right)$ does not introduce strong biases.

Table 1

This Table Summarizes the 10 Different Spectroscopic Classifications Used in This Paper

\begin{tabular}{|c|c|c|}
\hline Spectral Class & Expanded & Detailed Description \\
\hline PAS & passive & $\begin{array}{l}\text { Absorption line spectrum with no detected emission lines and } \mathrm{EW}\left(\overline{\mathrm{H}_{\delta \gamma \beta}}\right)>-3 \AA \text {, } \\
\text { indicating an old, passively evolving stellar population. }\end{array}$ \\
\hline sNSF & strong non-star-forming & As for NSF, but with $\mathrm{EW}(\mathrm{H} \alpha)>6 \AA$. \\
\hline wNSF & weak non-star-forming & As for NSF, but with $3 \AA<E W(H \alpha)<6 \AA$. \\
\hline rNSF & retired non-star-forming & As for NSF, but with $\mathrm{EW}(\mathrm{H} \alpha)<3 \AA$. \\
\hline wSF & weak star-forming & As for $\mathrm{SF}$, but with $\mathrm{EW}(\mathrm{H} \alpha)<3 \AA$. \\
\hline INT & intermediate & $\begin{array}{l}\text { Emission lines detected. Line ratios are intermediate between the SF and NSF } \\
\text { diagnostic boundaries. Emission likely due to composite of star-forming and } \\
\text { non-star-forming mechanisms. }\end{array}$ \\
\hline rINT & retired intermediate & As for INT, but with $\mathrm{EW}(\mathrm{H} \alpha)<3 \AA$. \\
\hline HDS & $\mathrm{H} \delta$-strong/post-star-forming & $\begin{array}{l}\text { Absorption line spectrum with no detected emission lines. } \\
\text { Strong Balmer absorption with } \operatorname{EW}\left(\overline{\mathrm{H}_{\delta \gamma \beta}}\right)<-3 \AA \text { indicating truncation } \\
\text { of star formation in last } \sim 1.5 \mathrm{Gyr} \text {. }\end{array}$ \\
\hline
\end{tabular}

with $\mathrm{S} / \mathrm{N}(4100 \AA) \sim 3 \mathrm{pix}^{-1}, \sigma_{\mathrm{EW}\left(\overline{\mathrm{H}_{\delta \gamma \beta}}\right)} \sim 1 \AA$, indicating that we can now reliably distinguish passive and HDS spectra even at low $\mathrm{S} / \mathrm{N}$.

\section{Spectroscopic Classification}

A key aim of this paper is to identify galaxies that are in the process of being quenched. This requires the identification of regions that contain young $(\lesssim 1.5 \mathrm{Gyr})$ stellar populations with no significant ongoing star formation. We identify these regions using a combination of emission and absorption line diagnostics, as described below. The 10 spectral classifications are summarized in Table 1 . We only include spaxels where the continuum $\mathrm{S} / \mathrm{N}$ is $\mathrm{S} / \mathrm{N}(4100 \AA)>3 \mathrm{pix}^{-1}$ to ensure that the continuum fits described in Section 3.1 are reliable and that both emission and absorption line classification are possible.

\subsection{Emission Line Classification}

In order to be considered for emission line classification, a spaxel must have either $\mathrm{H} \alpha$ or $[\mathrm{N}$ II] $(\lambda 6583)$, plus one of $\mathrm{H} \beta$, [O III $](\lambda 5007)$, [S II $](\lambda 6716)$, or $[\mathrm{S} \mathrm{II}](\lambda 6730)$ lines detected with $\mathrm{S} / \mathrm{N}>3$, where the $\mathrm{S} / \mathrm{N}$ of the line measurement is estimated as the ratio of the measured flux and its formal uncertainty. For both of these two scenarios, we also require that the primary line (i.e., $\mathrm{H} \alpha$ or $[\mathrm{N} \mathrm{II}](\lambda 6583)$ ) must have EW $>1 \AA$, which helps to reject spurious detections due to template mismatch. The detection of at least two lines with $\mathrm{S} / \mathrm{N}>3$ guards against the bias toward false-positive detections that are known to occur for single-line detections with $\mathrm{S} / \mathrm{N}<5$ (Rola \& Pelat 1994).

The standard procedure to classify emission line spectra is to use the line ratio diagrams of Baldwin et al. (1981, hereafter BPT) and Veilleux \& Osterbrock (1987), which plot the flux ratios for $[\mathrm{O} \mathrm{III}](\lambda 5007) / \mathrm{H} \beta$ versus $[\mathrm{N} \mathrm{II}](\lambda 6583) / \mathrm{H} \alpha$, 
Table 2

Summary of the Emission Line Classification Scheme Described in Section 4.1

\begin{tabular}{|c|c|c|c|c|}
\hline Category & $\begin{array}{l}\text { Detected Emission } \\
\text { Lines }(\mathrm{S} / \mathrm{N}>3)\end{array}$ & SF & $\begin{array}{c}\text { Classification Method } \\
\text { INT }\end{array}$ & NSF \\
\hline A & $\begin{array}{c}\mathrm{H} \beta,[\mathrm{O} \mathrm{III}](\lambda 5007), \\
\mathrm{H} \alpha,[\mathrm{N} \mathrm{III}]\end{array}$ & $\mathrm{P}(\mathrm{SF})>\mathrm{P}(\mathrm{INT}), \mathrm{P}(\mathrm{NSF})$ & $\mathrm{P}(\mathrm{INT})>\mathrm{P}(\mathrm{SF}), \mathrm{P}(\mathrm{NSF})$ & $\mathrm{P}(\mathrm{NSF})>\mathrm{P}(\mathrm{SF}), \mathrm{P}(\mathrm{INT})$ \\
\hline B & $\begin{array}{c}{[\mathrm{O} \text { III }](\lambda 5007), \mathrm{H} \alpha,} \\
{[\mathrm{N} \mathrm{III}]}\end{array}$ & $\begin{array}{c}\mathrm{P}(\mathrm{NSF})<0.9 \text { AND } \\
\log ([\mathrm{N} \text { II }] / \mathrm{H} \alpha)<-0.32\end{array}$ & $\begin{array}{c}\mathrm{P}(\mathrm{NSF})<0.9 \text { AND } \\
-0.32<\log ([\mathrm{N} \mathrm{II}] / \mathrm{H} \alpha)<0.1\end{array}$ & $\begin{array}{c}\mathrm{P}(\mathrm{NSF}) \geqslant 0.9 \text { OR } \\
(\mathrm{P}(\mathrm{NSF})<0.9 \text { AND } \\
\log ([\mathrm{N} \mathrm{II}] / \mathrm{H} \alpha)>0.1)\end{array}$ \\
\hline $\mathrm{C}$ & $\mathrm{H} \beta, \mathrm{H} \alpha,[\mathrm{N}$ II $]$ & $\begin{array}{c}\mathrm{P}(\mathrm{SF}) \geqslant 0.9 \text { OR } \\
(\mathrm{P}(\mathrm{SF})<0.9 \text { AND } \\
\log ([\mathrm{N} \mathrm{II}] / \mathrm{H} \alpha)<-0.32)\end{array}$ & $\begin{array}{c}\mathrm{P}(\mathrm{SF})<0.9 \text { AND } \\
-0.32<\log ([\mathrm{N} \mathrm{II}] / \mathrm{H} \alpha)<0.1 \\
-0.32<\log ([\mathrm{N} \mathrm{II}] / \mathrm{H} \alpha)<0.1\end{array}$ & $\begin{array}{c}\mathrm{P}(\mathrm{SF})<0.9 \text { AND } \\
\log ([\mathrm{N} \mathrm{II}] / \mathrm{H} \alpha)>0.1\end{array}$ \\
\hline $\mathrm{D}$ & $\mathrm{H} \alpha,[\mathrm{N} \mathrm{II}]$ & $\log ([\mathrm{N}$ II $] / H \alpha)<-0.32$ & $-0.32<\log ([\mathrm{N} \mathrm{II}] / \mathrm{H} \alpha)<0.1$ & $\log ([\mathrm{N} \mathrm{II}] / \mathrm{H} \alpha)>0.1$ \\
\hline $\mathrm{E}$ & $\begin{array}{c}(\mathrm{H} \alpha, \text { NOT [N II] }) \text { OR } \\
([\mathrm{N} \text { II] NOT H } \alpha)\end{array}$ & IF $\mathrm{H} \alpha$ & $\begin{array}{l}\cdots \\
\cdots\end{array}$ & IF [N II] \\
\hline Subclasses & $\cdots$ & wSF IF EW $(\mathrm{H} \alpha)<3 \AA$ & $\operatorname{rINT} \operatorname{IF} \operatorname{EW}(\mathrm{H} \alpha)<3 \AA$ & $\begin{array}{c}\text { SNSF IF } \operatorname{EW}(\mathrm{H} \alpha)>6 \AA \\
\text { wNSF IF } 3 \AA<\operatorname{EW}(\mathrm{H} \alpha)<6 \AA \\
\text { rNSF IF } \mathrm{EW}(\mathrm{H} \alpha)<3 \AA\end{array}$ \\
\hline
\end{tabular}

Note. The probabilities listed for category A, B, and C spectra are determined using 5000 Monte Carlo realizations of the [N II] $(\lambda 6583) / \mathrm{H} \alpha$ and $[\mathrm{O}$ III] $(\lambda 5007) / \mathrm{H} \beta$ line ratios and determining the fraction that fall in the SF, INT, and NSF regions of the BPT diagram defined by the demarcation lines of Kauffmann et al. (2003c) and Kewley et al. (2001). Category B, C, and D spectra may also be classified on the basis of the two-line scheme of Cid Fernandes et al. (2010). Subclasses based on $\mathrm{EW}(\mathrm{H} \alpha)$ are also listed, where "r" stands for retired, "w" stands for weak, and "s" stands for strong.

$[\mathrm{S}$ II $](\lambda \lambda 6716,6731) / \mathrm{H} \alpha$, or $[\mathrm{OI}](\lambda 6300) / \mathrm{H} \alpha$. Generally, a $\mathrm{S} / \mathrm{N}$ cut is made on each of the four lines involved in the line ratio diagram so that their positions on BPT diagrams can be reliably measured (e.g., Kewley et al. 2006). However, these more conservative cuts prohibit classification for a large number of emission line spaxels where fewer than four lines are detected, and may bias against particular types of emission line galaxies (Miller et al. 2003; Cid Fernandes et al. 2010). Given these issues, and because our aim is to search for signatures of recent star formation in the absence of ongoing star formation, it is very important that we are able to characterize any emission detected in a spaxel as arising from a SF or non-SF (NSF) ionizing source even when only a subset of the BPT lines are detected. Spaxels that meet the emission line classification may lie in five different categories depending on the combination of emission lines that are detected with $\mathrm{S} / \mathrm{N}>3$ :

1. Category A: All four of the $\mathrm{H} \alpha$, [N II] ( $\lambda 6583), \mathrm{H} \beta$, and [O III $](\lambda 5007)$ lines are detected;

2. Category B: $\mathrm{H} \alpha$, $[\mathrm{N}$ II $]$ ( $\lambda 6583)$, and $[\mathrm{O} \mathrm{III}](\lambda 5007)$ lines are detected, but $\mathrm{H} \beta$ is not;

3. Category $C$ : $\mathrm{H} \alpha,[\mathrm{NII}](\lambda 6583)$, and $\mathrm{H} \beta$ lines are detected, but $[\mathrm{O} \mathrm{III}](\lambda 5007)$ is not;

4. Category D: $\mathrm{H} \alpha$ and $[\mathrm{N} \mathrm{II}]$ ( $\lambda 6583)$ lines are detected, but $\mathrm{H} \beta$ and $[\mathrm{O} \mathrm{III}](\lambda 5007)$ are not;

5. Category $E: \mathrm{H} \alpha$ and one other line that is not [N II] $(\lambda 6583)$ are detected, or $[\mathrm{N} \mathrm{II}](\lambda 6583)$ and one other line that is not $\mathrm{H} \alpha$ are detected.

The emission line classification scheme for each of these five categories is summarized in Table 2. A detailed explanation of the emission line classification scheme follows.

Spectra that fall into categories $\mathrm{A}-\mathrm{C}$ are classified in a probabilistic manner using the $[\mathrm{N} \mathrm{II}](\lambda 6583) / \mathrm{H} \alpha$ versus
[O III $](\lambda 5007) / \mathrm{H} \beta$ BPT diagram (similar to the methods of Carter et al. 2001; Manzer \& De Robertis 2014; Marziani et al. 2017). We produce $5000 \mathrm{MC}$ realizations of the $[\mathrm{N} \mathrm{II}] / \mathrm{H} \alpha$ and $[\mathrm{O} \mathrm{III}] / \mathrm{H} \beta$ ratios, assuming a Gaussian distribution centered at the measured line flux with dispersion equal to the flux uncertainty. We include a contribution due to uncertainty on the emission line flux caused by the SPS template-based absorption correction for $\mathrm{H} \beta$ and $\mathrm{H} \alpha$, which is assumed to be $0.5 \AA$ in equivalent width, consistent with the typical uncertainty on the $\operatorname{EW}(\mathrm{H} \beta)$ measured in Section 3.3. We classify each $\mathrm{MC}$ realization according to its position in the BPT diagram using the regions defined by Kewley et al. (2006). MC realizations that have $[\mathrm{N} \mathrm{II}] / \mathrm{H} \alpha$ and $[\mathrm{O} \mathrm{IIII}] / \mathrm{H} \beta$ ratios that place them: (i) below the empirically based Kauffmann et al. (2003a) demarcation are classified as SF, (ii) above the Kauffmann et al. (2003a) and below the Kewley et al. (2001) theoretical "maximum starburst" demarcation lines are classified as intermediate/composite (INT), and (iii) above the Kewley et al. (2001) demarcation are classified as NSF. We then use the fraction of MC realizations falling into the three separate BPT classifications to determine the probabilities $\mathrm{P}(\mathrm{SF}), \mathrm{P}(\mathrm{INT})$, and $\mathrm{P}(\mathrm{NSF})$. Spectra that fall into category A are classified using the BPT class that has the highest probability.

For the category A spectra, the probabilistic classification is identical to classifying spectra according to the ratios derived from the measured emission line flux values, assuming the probability density distribution is symmetric about the measured line ratios. This method becomes more powerful when considering category $\mathrm{B}$ and $\mathrm{C}$ spectra, where judicious use of upper limits can enable a classification in the absence of a significant line detection for $\mathrm{H} \beta$ or $[\mathrm{O}$ III $](\lambda 5007)$. For the category B galaxies, we can place an upper limit on the $\mathrm{H} \beta$ line flux based on the $\mathrm{H} \alpha$ line flux and our knowledge of case-B recombination, which results in $F_{\mathrm{H} \beta}<F_{\mathrm{H} \alpha} / 2.86$. During the 
MC realizations, we enforce this upper limit. The upper limit on $F_{\mathrm{H} \beta}$ enables a lower limit to be placed on the $[\mathrm{O}$ III $](\lambda 5007) / \mathrm{H} \beta$ line ratio and allows us to robustly classify spectra as lying above the Kewley et al. (2001) demarcation line, thereby ruling out INT and SF classifications. We can therefore classify any category B spectrum as NSF, although we use a more conservative cutoff of $\mathrm{P}(\mathrm{NSF})>0.9$. Likewise, for category $\mathrm{C}$ spectra we can determine upper limits on the $[\mathrm{O} \mathrm{III}](\lambda 5007)$ line flux on the basis of the flux uncertainties, which enables an upper limit on the $[\mathrm{O} \mathrm{III}](\lambda 5007) / \mathrm{H} \beta$ line ratio to be determined. Category $\mathrm{C}$ spectra are classified as lying below the Kauffmann et al. (2003a) demarcation line when $\mathrm{P}(\mathrm{SF})>0.9$.

Category D spectra are classified on the basis of the $[\mathrm{N} \mathrm{II}](\lambda 6583) / \mathrm{H} \alpha$ ratio using the demarcation lines derived by Cid Fernandes et al. (2010). The boundaries used for the SF, INT, and NSF classifications are shown in Table 2 . The divisions at log $([\mathrm{N}$ II $](\lambda 6583) / \mathrm{H} \alpha)=-0.32$ and $\log ([\mathrm{N} \mathrm{II}](\lambda 6583) / \mathrm{H} \alpha)=$ -0.1 correspond to the optimal dividing lines for the Kauffmann et al. (2003a) and Kewley et al. (2001) BPT demarcation lines, as determined in Cid Fernandes et al. (2010). These divisions are chosen to be consistent with the classification scheme outlined for category A galaxies. Those category B and $\mathrm{C}$ spectra that could not be robustly classified as NSF or SF, respectively, were also classified using this method. Category E spectra, where the $\mathrm{H} \alpha$ line is detected and $[\mathrm{N} \mathrm{II}](\lambda 6583)$ is not, are classified as SF, while those where the $[\mathrm{N} \mathrm{II}](\lambda 6583)$ line is detected with no $\mathrm{H} \alpha$ detection are classified as NSF.

In the above classifications, we have thus far only made use of line flux ratios. Cid Fernandes et al. (2011) advocated for the combined use of line ratios and the $\mathrm{EW}(\mathrm{H} \alpha)$ when performing spectroscopic classification, particularly when only a subset of emission lines are detected. In particular, they classify spectra with $\mathrm{EW}(\mathrm{H} \alpha)<3 \AA$ as being retired because the emission is likely powered by ionization driven by post-asymptotic giant branch stars (Cid Fernandes et al. 2011; Singh et al. 2013; Belfiore et al. 2016, 2017). We incorporate the $\mathrm{EW}(\mathrm{H} \alpha)$ into our classifications in a manner similar to that of Cid Fernandes et al. (2011). Spectra that have $\mathrm{EW}(\mathrm{H} \alpha)<3 \AA$, are categorized into the subcategories rINT, rNSF, and wSF, where the " $r$ " stands for retired (following the Cid Fernandes et al. 2011 nomenclature), and the "w" stands for weak, because the line ratios implies there may be star formation present but the low $\operatorname{EW}(\mathrm{H} \alpha)$ indicates that it is relatively weak. We subcategorize those NSF galaxies with $3 \AA<\mathrm{EW}(\mathrm{H} \alpha)<6 \AA$ as wNSF and those with $\mathrm{EW}(\mathrm{H} \alpha) \geqslant 6 \AA$ as sNSF, where "s" stands for strong.

\subsection{Absorption Line Classification}

Those spectra that do not have at least two emission lines detected as outlined in Section 4.1 are classified as absorption line spectra. Absorption line spectra are further classified according to the strength of $\mathrm{EW}\left(\overline{\mathrm{H}_{\delta \gamma \beta}}\right)$. This classification is performed in a manner similar to that described in Dressler et al. (1999) and Poggianti et al. (1999), where the strength of the age-sensitive $\mathrm{H} \delta$ line was used as a proxy to identify passively evolving spectra, as well as those showing recently truncated star formation. We classify spectra with $\mathrm{EW}\left(\overline{\mathrm{H}_{\delta \gamma \beta}}\right)<-3 \AA$ and $\mathrm{S} / \mathrm{N}\left(\mathrm{EW}\left(\overline{\mathrm{H}_{\delta \gamma \beta}}\right)\right)>3$ as HDS spectra and those absorption line spectra not meeting this criteria as passive. We choose this limit in $\mathrm{EW}\left(\overline{\mathrm{H}_{\delta \gamma \beta}}\right)$ on the basis of (i) data limitations - at our limiting $\mathrm{S} / \mathrm{N}(4100 \AA)=3 \mathrm{pix}^{-1}$ the median error on $\mathrm{EW}\left(\overline{\mathrm{H}_{\delta \gamma \beta}}\right)$ is $\sim 1 \AA$, meaning we can relatively reliably measure $\mathrm{EW}\left(\overline{\mathrm{H}_{\delta \gamma \beta}}\right)$ for our spectra; and (ii) spectra exhibiting $\mathrm{H} \delta$ absorption stronger than this limit generally only occur because of a recent truncation of star formation (as opposed to a slow decline in star formation), as discussed in Poggianti et al. (1999).

We stress here that we are not searching for poststarburst signatures, which would require a more stringent $\mathrm{EW}\left(\overline{\mathrm{H}_{\delta \gamma \beta}}\right)<$ $-5 \AA$ criterion as used in other studies (e.g., Couch \& Sharples 1987; Zabludoff et al. 1996; Blake et al. 2004). Only around $5 \%$ of the HDS-classified spectra in our sample would meet this more stringent criterion. Rather, our criteria allow us to robustly identify spectra that are likely to have experienced a recent truncation of star formation within the last $\sim 1.5 \mathrm{Gyr}$ (i.e., post-SF regions), regardless of whether that truncation was preceded by a starburst.

In addition to the absorption line spectra that are classified as HDS, we add another HDS classification for those spectra that were classified as NSF in Section 4.1, but also have $\mathrm{EW}\left(\overline{\mathrm{H}_{\delta \gamma \beta}}\right)<-3 \AA$. These spectra are labeled NSF_HDS; they meet the criteria of having evidence for young stellar populations with no ongoing star formation (similar to the poststarburst galaxies in other studies; Yan et al. 2006; Alatalo et al. 2016). Here, we add the additional criterion that the flux of $\mathrm{H} \delta$ in emission must not exceed half the flux in absorption (as determined from the emission line free spectrum). This criterion is somewhat arbitrary but ensures that spectra where the strong emission completely masks the Balmer absorption are not classified as HDS. This complete masking can occur in regions of strong AGN emission, and, in these cases, the absorption line measurements are strongly dependent on the correct modeling of the underlying stellar continuum and may lead to spurious $\mathrm{EW}\left(\overline{\mathrm{H}_{\delta \gamma \beta}}\right)$ measurements.

\section{Galaxy Classification Scheme}

In this section, we use the resolved spectroscopic classifications from Section 4 to divide our sample into PASGs, SFGs, and HDSGs.

\subsection{What are Passive and Star-forming Spaxels?}

Many of the spectroscopic classifications defined in Section 4 are readily associated with passive stellar populations, ongoing star formation, or recently truncated star formation. For absorption line spectra, the distinction is, by construction, trivial: the HDS spectra represent recently truncated, post-SF regions, and the remainder, which show no strong Balmer absorption, are associated with older, passively evolving stellar populations. Similarly, spectra with strong emission lines with flux ratios placing them in the SF class are clearly associated with regions with ongoing star formation.

However, for other classes of emission line galaxies (e.g., INT, rINT, or wSF classifications), it is not always obvious whether a spectrum should be classed as passive or SF. To help with further classification, we investigate the distribution of the various spectral types in the $\mathrm{EW}\left(\overline{\mathrm{H}_{\delta \gamma \beta}}\right)-\mathrm{D}_{\mathrm{N}} 4000$ plane, where $\mathrm{D}_{\mathrm{N}} 4000$ is the $4000 \AA$ break strength, which is determined using the definition of Balogh et al. (1999). The position on the $\mathrm{EW}\left(\overline{\mathrm{H}_{\delta \gamma \beta}}\right)-\mathrm{D}_{\mathrm{N}} 4000$ plane is a relatively reliable proxy for the luminosity-weighted age of the underlying stellar population. Young stellar populations inhabit regions with strong $\mathrm{EW}\left(\overline{\mathrm{H}_{\delta \gamma \beta}}\right)$ absorption and weaker breaks at $\mathrm{D}_{\mathrm{N}} 4000$, while 

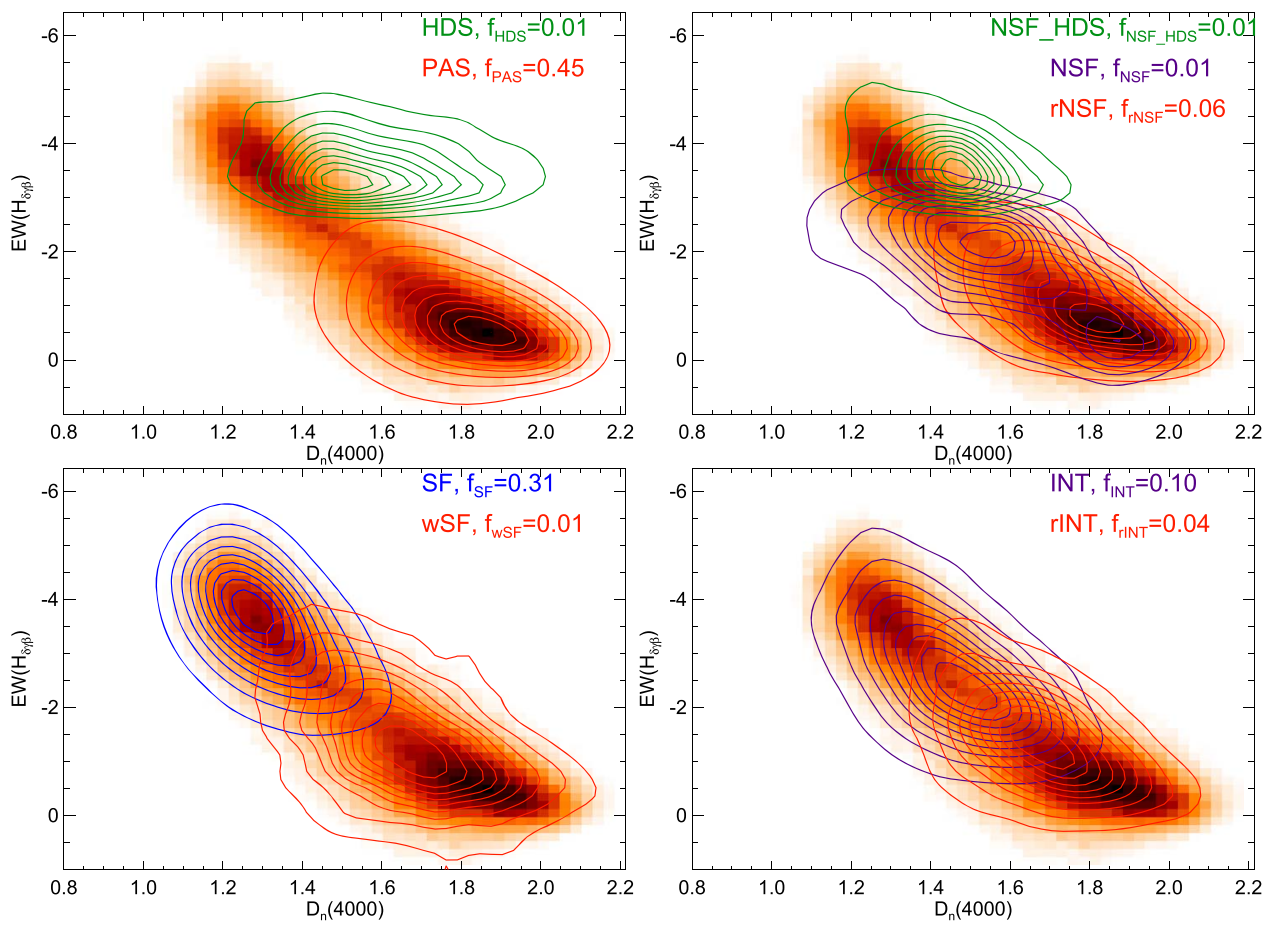

Figure 4. Distribution of $\mathrm{EW}\left(\overline{\mathrm{H}_{\delta \gamma \beta}}\right)$ vs. $\mathrm{D}_{\mathrm{N}} 4000$ for the spectral classifications defined in Sections 4.1 and 4.2. The green and red contours in the top left panel show the distribution for spaxels classified as HDS and PAS, respectively. The top right panel shows the emission spaxels where the emission was classified as NSF: green shows NSF_HDS, purple NSF (sNSF plus wNSF), and red rNSF. The bottom left panel shows the distribution for emission line spaxels classified as either SF in (blue contours) or wSF (red contours). The bottom right panel shows the distributions for the rINT (red) and INT (purple) spectra. The heat map in each plot shows the number density of the $\mathrm{D}_{\mathrm{N}} 4000$ vs. $\mathrm{EW}\left(\overline{\mathrm{H}_{\delta \gamma \beta}}\right)$ distribution for all spaxels with $\mathrm{S} / \mathrm{N}(4100 \AA)>3$ pix $^{-1}$ and for cluster and GAMA galaxies with $\log \left(M_{*} / M_{\odot}\right) \geqslant 10$. The fractional contribution of each of the different spectral classifications is shown in the top right of each plot. The majority of spectra are classified as SF or PAS, which make up $31 \%$ and $45 \%$ of classified spectra, respectively. The HDS and NSF_HDS spectra are rare, contributing to only $2 \%$ of the classified spectra.

older, passively evolving stellar populations inhabit regions with stronger $\mathrm{D}_{\mathrm{N}} 4000$ and weaker $\mathrm{EW}\left(\overline{\mathrm{H}_{\delta \gamma \beta}}\right)$ (Balogh et al. 1999; Kauffmann et al. 2003c).

Figure 4 shows the number density distribution of the $\mathrm{EW}\left(\overline{\mathrm{H}_{\delta \gamma \beta}}\right)$ as a function of $\mathrm{D}_{\mathrm{N}} 4000$ for all classifiable spectra in the cluster and GAMA samples that have $\mathrm{S} / \mathrm{N}(4100 \AA)$ $>3 \mathrm{pix}^{-1}$. The four panels in Figure 4 show the $\mathrm{EW}\left(\overline{\mathrm{H}_{\delta \gamma \beta}}\right)-\mathrm{D}_{\mathrm{N}} 4000$ distribution for each of the spectroscopic subclassifications in the absorption line (top left panel), the NSF (top right panel), SF (lower left panel), and INT (lower right) classes. In these panels, the $\mathrm{EW}\left(\overline{\mathrm{H}_{\delta \gamma \beta}}\right)-\mathrm{D}_{\mathrm{N}} 4000$ distributions for the subclasses are shown as nine equally spaced contours that range from $10 \%$ to $90 \%$ of the peak in the smoothed number density for the spectral type of interest. Figure 4 reveals that there are two clear peaks in the $\mathrm{EW}\left(\overline{\mathrm{H}_{\delta \gamma \beta}}\right)-\mathrm{D}_{\mathrm{N}} 4000$ plane: one centered at $\mathrm{EW}\left(\overline{\mathrm{H}_{\delta \gamma \beta}}\right) \simeq$ $-0.5 \AA$ and $\mathrm{D}_{\mathrm{N}} 4000 \simeq 1.85$ and the other at $\mathrm{EW}\left(\overline{\mathrm{H}_{\delta \gamma \beta}}\right) \simeq$ $-4.0 \AA$ and $\mathrm{D}_{\mathrm{N}} 4000 \simeq 1.30$. The former peak is dominated by spaxels classified as PAS (red contours in the top left panel), which make up $45 \%$ of all classified spaxels, while the latter peak primarily contains spaxels classified as SF (blue contours in the bottom left panel), which make up $31 \%$ of classified spaxels.

The $\mathrm{EW}\left(\overline{\mathrm{H}_{\delta \gamma \beta}}\right)-\mathrm{D}_{\mathrm{N}} 4000$ distributions of the rNSF, wSF, and rINT classified emission line spaxels (all of which have weak $\mathrm{EW}(\mathrm{H} \alpha)<3 \AA$ ) are shown as red contours in the top right, bottom left, and bottom right panels of Figure 4, respectively. The distributions of these weak $\mathrm{H} \alpha$ emitters are generally consistent with that of the absorption line PASGs, indicating that the stellar populations in these spectra are dominated by old, passive populations. It is interesting to note that even wSF classified spectra are more consistent with passively evolving stellar populations, although there is a small fraction of wSF spectra that occupy regions consistent with recent star formation. We therefore conclude that the rNSF, wSF, and rINT spectral types are to be considered alongside the passive type as being dominated by passively evolving, old stellar populations. Because of their low numbers, wNSF and SNSF classified spaxels are combined, and their $\mathrm{EW}\left(\overline{\mathrm{H}_{\delta \gamma \beta}}\right)-\mathrm{D}_{\mathrm{N}} 4000$ distribution is shown as purple contours in the top right panel of Figure 4. There is no strong indication that the NSF spaxels are dominated by young stellar populations. Given the NSF origin of the emission in these spaxels, we also count them as passive spaxels.

The purple contours in the lower right panel in Figure 4 show the distribution of emission line spaxels that are classified as INT. For INT spectra, the peak in the distribution is located between the SF and passive peaks and extends to encompass the peak associated with SF-classified spaxels, indicating that a large fraction of INT spectra harbor young stellar populations as a result of recent or ongoing star formation. INT-type spectra are often interpreted as being due to the combination of emission that has been ionized by both star formation and NSF processes (e.g., AGN; Kewley et al. 2006). This interpretation is supported by the large fraction of INT spaxels that show evidence for young stellar population in Figure 4. We therefore include those INT spectra as SF along with the SF-classified spaxels. 


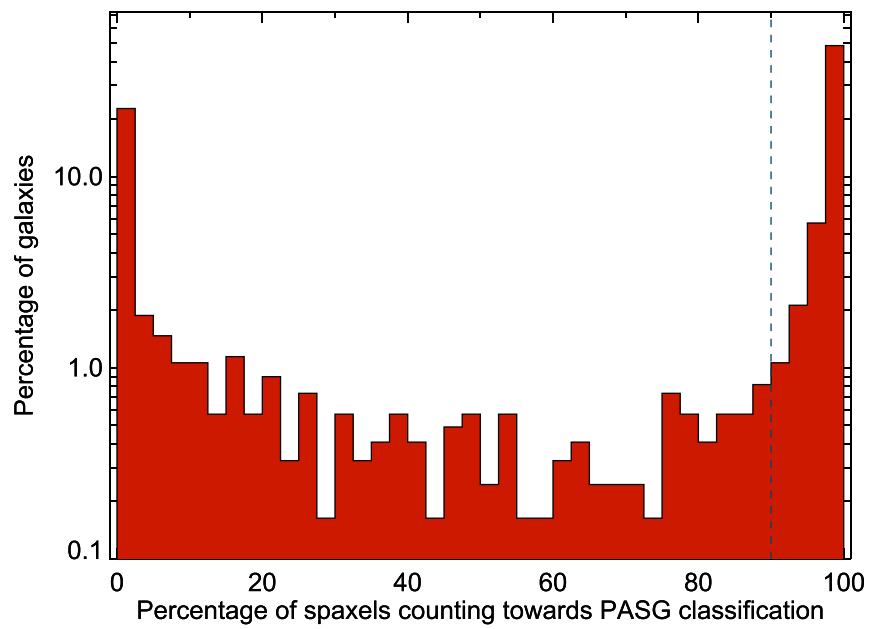

Figure 5. Percentage of galaxies in the sample (on a log scale) as a function of the percentage of classifiable spaxels associated with passively evolving stellar populations (i.e., spectroscopic classes passive, rNSF, wNSF, sNSF, rINT, wSF, from Section 5). This plot shows that for $54 \%$ of galaxies in the sample, $\geqslant 95 \%$ of spaxels with $\mathrm{S} / \mathrm{N}(4100 \AA)>3 \mathrm{pix}^{-1}$ have spectral types associated with passively evolving stellar populations. The dashed line shows our dividing line at $90 \%$, above which a galaxy is classified as PASG. The remaining galaxies with fewer than $90 \%$ passive spaxels will be classified as either HDSG or SFGs.

\subsection{How Many Spaxels Define a Galaxy Class?}

We take a pragmatic approach to determining the fraction of spaxels associated with passively evolving stellar populations that are required for a galaxy to be classified as PASG. In Figure 5 we present a histogram that shows the relative frequency of the fraction of passive spaxels for SAMI galaxies in our sample. In determining the fraction of passive spaxels, only those spaxels with $\mathrm{S} / \mathrm{N}(4100 \AA)>3 \mathrm{pix}^{-1}$ are used. Figure 5 demonstrates that a large fraction of our sample is dominated by passive spaxels; $54 \%$ of the galaxies have $\geqslant 95 \%$ of their spaxels belonging to spectroscopic classes that are associated with passively evolving stellar populations (i.e., those with passive, NSF, rINT, and wSF). This fraction only increases to $57 \%$ when considering galaxies with $\geqslant 90 \%$ of spaxels associated with passively evolving spectroscopic classes. This convergence at $90 \%$ therefore sets a natural lower limit on the fraction of passive spaxels required for a galaxy to fall into the PASG class. Conversely, the limit for the PASG class also sets the lower limit of $10 \%$ of spaxels classified as INT or SF for the SFG class. Likewise, a HDSG must have at least $10 \%$ of its spaxels classified as HDS or NSF_HDS. To summarize, our galaxy classes are defined as:

1. PASG: passive galaxies that have more than $90 \%$ of $\mathrm{S} / \mathrm{N}$ $(4100 \AA)>3$ pix $^{-1}$ spaxels classified as passive, rNSF, rINT, wNSF, sNSF, or wSF.

2. SFG: star-forming galaxies have $10 \%$ or more $\mathrm{S} / \mathrm{N}$ $(4100 \AA)>3$ pix $^{-1}$ spaxels classified as either INT or SF.

3. HDSG: $\mathrm{H} \delta$-strong galaxies have $10 \%$ or more $\mathrm{S} / \mathrm{N}$ $(4100 \AA)>3$ pix $^{-1}$ spaxels classified as either HDS or NSF_HDS.

In addition, for the SFG and HDSG classes we introduce a continuity criterion in order for a spaxel to contribute to the $10 \%$ limit. For the SFG class, three of the six spaxels surrounding an INT or SF spaxel must also be classified as INT or SF. Likewise, to count toward HDSG classification, three of the six pixels surrounding an HDS or NSF_HDS spaxel must be classified as HDS or NSF_HDS. This guards against the contribution of isolated spaxels that can occur by chance in lower $\mathrm{S} / \mathrm{N}$ spectra. We note that a galaxy may simultaneously meet the criteria for the SFG and HDSG classes, and in these cases the galaxy is included in the HDSG sample.

For the subset of 1220 SAMI targets used in this paper, the majority $(88 \%)$ of the sample contains 100 or more spaxels with $\mathrm{S} / \mathrm{N}(4100 \AA)>3 \mathrm{pix}^{-1}$. Therefore, a minimum of 10 spaxels are required to show evidence for recent/ongoing star formation in order for a galaxy to be classified as a SFG or HDSG. The median PSF of the SAMI survey has FWHM 2!.06 (Scott et al. 2018), which corresponds to a $1 \sigma$ surface area of 10 spaxels. Our criteria therefore ensure that, for a galaxy to be classified as a SFG or HDSG, the total area covered by the SF and HDS spaxels must be more extended than the PSF.

To check the veracity of our galaxy classification, we present Figure 6, which shows the observed, Galactic extinctioncorrected NUV $-r$ color versus stellar mass diagram for the cluster regions (left panel) and the GAMA regions (right panel). The NUV- $r$ colors for the GAMA galaxies are obtained from the LambdarPhotometry catalog released as part of the GAMA DR3 (Baldry et al. 2018), ${ }^{25}$ which provides magnitudes measured via the aperture-matched and deblended photometry described in Wright et al. (2016). The cluster NUV magnitudes come from the catalogs produced by Seibert et al. (2012). ${ }^{26}$ Each point is color-coded according to the fraction of $\mathrm{S} / \mathrm{N}(4100 \AA)>3 \mathrm{pix}^{-1}$ spaxels that are classified as passive, with solid circles, stars, and hexagons showing galaxies classified as PASG, SFG, and HDSG, respectively. Both the cluster and GAMA regions show a well-defined red-sequence that is predominantly populated by PASGs, as well as a blue cloud that is dominated by SFGs. The HDSGs generally lie blueward of the red-sequence.

\section{Results}

With the galaxy classifications at hand, we now focus on investigating the demographics of the HDSGs. Our primary aim here is to determine whether there are any correlations with measures of environment that may indicate that external influences are responsible for the shutdown of star formation in these systems.

\subsection{Comparison of HDSGs in the GAMA and Cluster Regions}

As a first-order proxy for environment, we compare the fraction of HDSGs found in the GAMA and cluster regions. The GAMA regions are primarily composed of galaxies that are either isolated or in groups with $\log \left(M_{200} / M_{\odot}\right)<14$. The HDSGs are rare overall in both the GAMA and cluster regions of the SAMI-GS, making up only $1.2_{-0.5}^{+0.6 \%}(8 / 647)$ and $3.0_{-0.6}^{+0.9} \%(17 / 575)$ of each sample, respectively. However, these fractions must be considered in light of the makeup of the galaxies in the two samples. The cluster sample is dominated by PASGs, which make up $80_{-2}^{+2} \%(460 / 575)$ of the sample, while the GAMA sample is dominated by SFGs, which make up $59_{-2}^{+2} \%(379 / 647)$ of the sample. If we consider the

\footnotetext{
25 http://www.gama-survey.org/dr3/

26 https://archive.stsci.edu/prepds/gcat/
} 

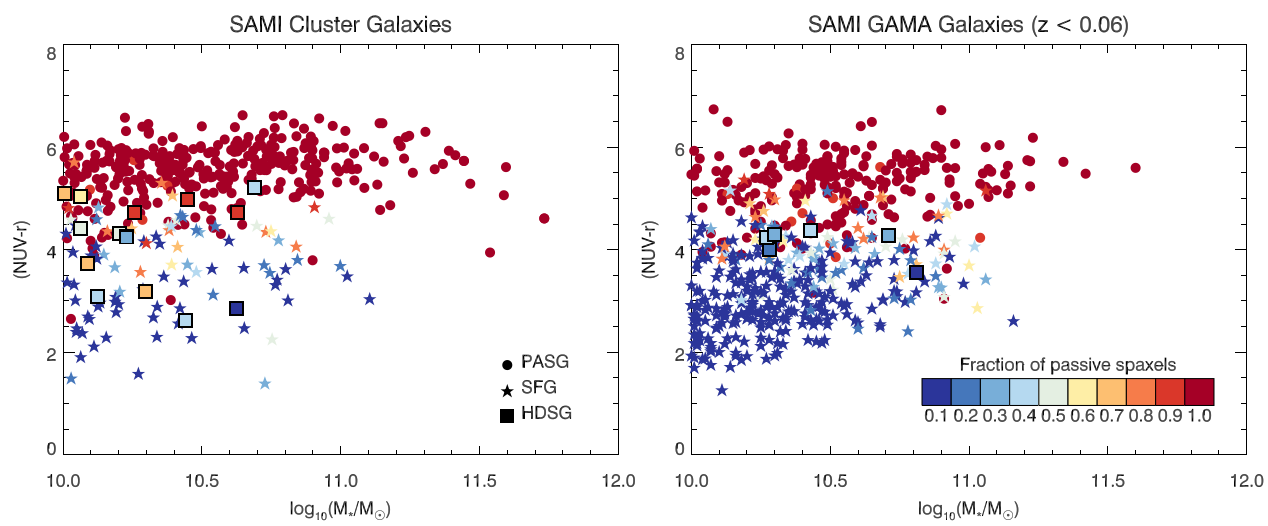

Figure 6. Color-mass diagrams for SAMI galaxies in the clusters (left panel) and GAMA regions (right panel). The color-coding of each point indicates the fraction of classifiable spaxels that have passively evolving stellar populations per the definition in Section 5.1. The three different galaxy classes are shown as different shapes, as indicated by the key in the bottom right of the left panel. Note that only the subset of cluster and GAMA galaxies that have NUV detections are plotted, and the NUV- $r$ colors are not $k$-corrected. These two figures indicate that the galaxy classification scheme outlined in Section 5.2 does a very good job of separating passive, redsequence galaxies and blue-cloud galaxies that are actively forming stars. Also of note is that, while the HDSGs have bluer colors than the passive red-sequence galaxies, they are generally found in the green valley.

"quenching efficiency," similar to that defined by Poggianti et al. (2009), which measures the fractional contribution of HDSGs to the population of galaxies that show evidence for recent star formation (i.e., $Q_{\mathrm{eff}}=N_{\mathrm{HDSG}} /\left(N_{\mathrm{SFG}}+N_{\mathrm{HDSG}}\right){ }^{27}$ for the cluster regions we find $Q_{\text {eff }}=15_{-3}^{+4} \%$, which is significantly higher than the $Q_{\text {eff }}=2.0_{-0.4}^{+1.0} \%$ found in the GAMA regions. All quoted uncertainties are $68 \%$ confidence intervals determined using the method described in Cameron (2011). This result strongly indicates that the cluster environment is much more efficient at quenching star formation when compared with the lower density environments found in the GAMA regions.

Aside from the difference in the quenching efficiency, there are three striking differences between the HDSGs found in the cluster regions when compared with those found in the GAMA regions that point to a distinct origin for the two populations. First, the GAMA HDSGs are not associated with massive groups; only one HDSG resides in a group with six members and $\log \left(M_{200} / M_{\odot}\right) \sim 13$. Of the remaining seven GAMA HDSGs, five have one or two neighboring galaxies within $100 \mathrm{kpc}$ and $100 \mathrm{~km} \mathrm{~s}^{-1}$, and two are completely isolated (i.e., there are no associated neighbors in the GAMA group catalogs of Robotham et al. 2011). That the GAMA HDSGs are not associated with massive groups in the GAMA regions strongly suggests that they are not being quenched by processes associated with cluster- or group-scale environmental influences.

Second, both the spatial distribution of the HDS regions and the nature of the emission lines differ when comparing the cluster and GAMA HDSGs. The differences are highlighted in Figure 7 (clusters) and 8 (GAMA), where the left-most panel shows the gri-band composite red, green, blue images; the top row of the right-most panel shows maps of the $\mathrm{EW}(\mathrm{H} \alpha), \log$ $([\mathrm{N} \mathrm{II}](\lambda 6583) / \mathrm{H} \alpha)$, and spectroscopic classification (top row); and the bottom row of the right-most panel shows the map of the $\mathrm{EW}\left(\overline{\mathrm{H}_{\delta \gamma \beta}}\right)$, as well as three example spectra. The example spectra are formed from the coaddition of the spaxels within

\footnotetext{
27 We note that this definition for quenching efficiency differs from that used in other studies, where the excess of completely quenched galaxies is measured relative to the field (e.g., Peng et al. 2010; Darvish et al. 2016; van der Burg et al. 2018).
}

$0.5 r_{e}$ (bottom spectrum), $0.5-1 r_{e}$ (middle spectrum), and from those spaxels classified as HDS or NSF_HDS (top spectrum).

The spectroscopic classification maps in Figure 7 reveal that more than half $(9 / 17)$ of the HDSGs in the clusters harbor evidence for $\mathrm{H} \alpha$ emission because of ongoing star formation within the central $0.5 r_{e}$ of the galaxy. On the other hand, the spectroscopic classification maps in Figure 8 show that only one of the eight GAMA HDSGs has evidence for ongoing star formation in its center. The emission associated with the other seven GAMA HDSGs is often classified as being due to AGN or shock ionization that is not associated with ongoing star formation, similar to those described in Alatalo et al. (2016). Considering the distribution of the HDS regions, Figure 7 shows that, in 14 of 17 cluster HDSGs, the HDS regions are found in the outer parts of the galaxy beyond $0.5 r_{e}$. Inspection of Figure 8 reveals that the HDS regions are far more evenly distributed throughout the GAMA HDSGs, where often the central $1 r_{e}$ is dominated by NSF_HDS-classified spaxels. The fact that the cluster HDSGs often exhibit central star formation, with HDS regions found in the outer parts of the galaxies, indicates that their star formation is being quenched in an outside-in manner. Contrastingly, the more evenly spread HDS regions found in the GAMA HDSGs, coupled with the evidence for shock-like and AGN emission associated with the HDS regions, indicates that the quenching of star formation may be a galaxy-wide event.

Third, the structure of the GAMA HDSGs is different from that of the cluster HDSGs. Figure 9 shows the distribution of the Sérsic index, $n_{\text {ser }}$, for the cluster and GAMA galaxies divided into the three galaxy classes. For both the GAMA and cluster samples, the distribution of $n_{\text {ser }}$ for SFGs and PASGs peaks at $n_{\text {ser }} \simeq 1-1.5$ and $n_{\text {ser }} \simeq 3-4$, respectively. The distributions of $n_{\text {ser }}$ are consistent with the expectation that the SFGs are disk dominated, while the PASGs are bulge dominated. Of the cluster HDSGs, $\sim 76 \%$ have $n_{\text {ser }}<2$ indicating that the majority of cluster HDSGs are disk dominated. The $n_{\text {ser }}$ distribution for the cluster HDSGs is consistent with the cluster SFGs; a Kolmogorov-Smirnov (KS) test does not reject the null hypothesis that the two distributions are drawn from the same parent population, returning a probability $P=0.15$. On the other hand, the majority $(7 / 8)$ of the GAMA HDSGs have $n_{\text {ser }}>2$. On comparing the 

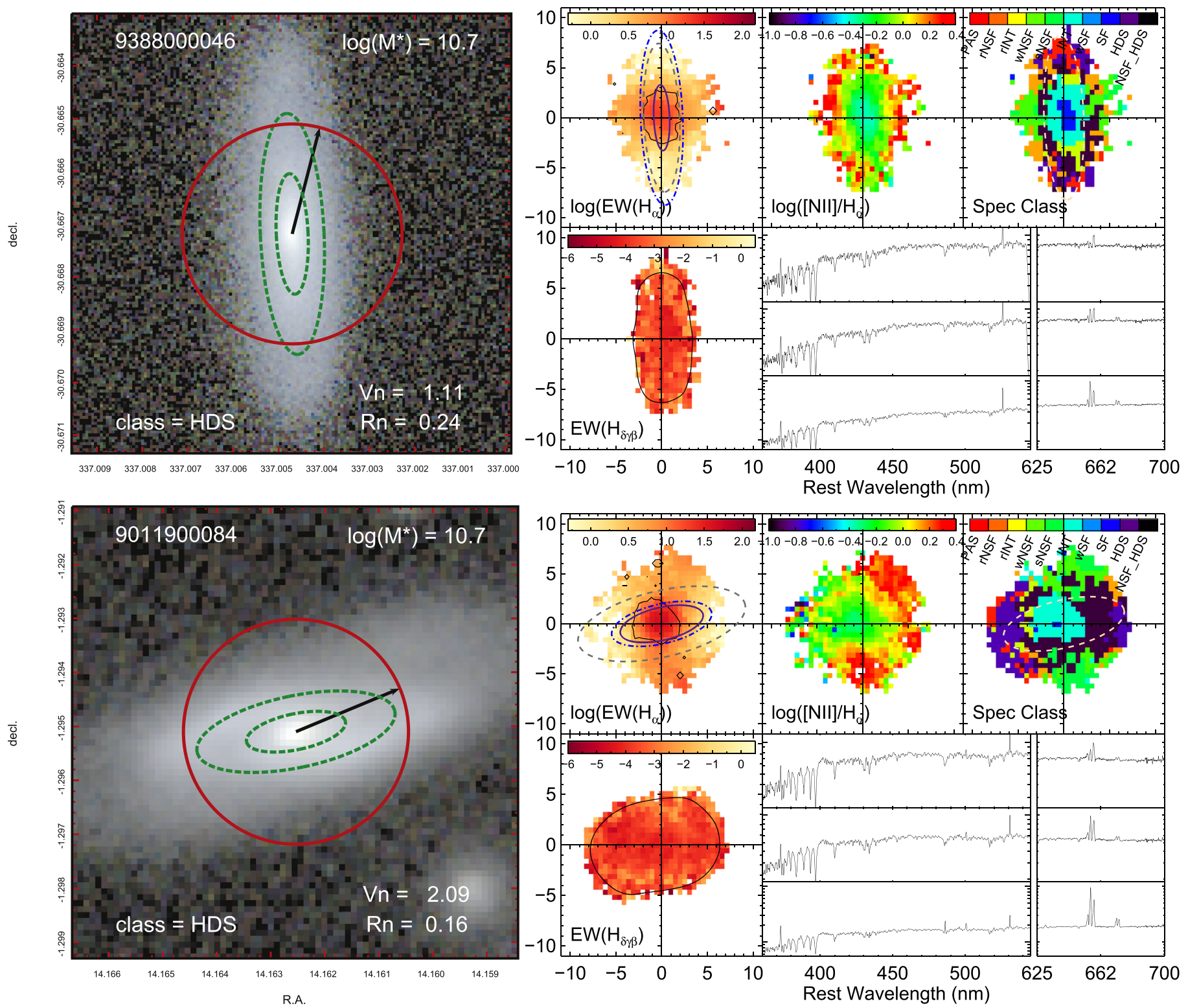

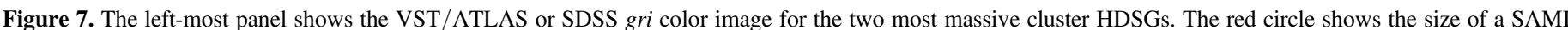

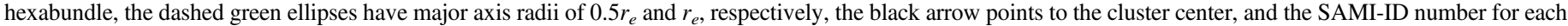

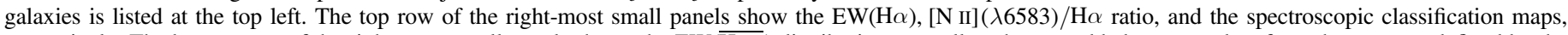

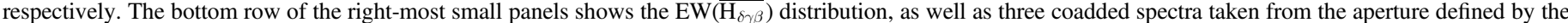

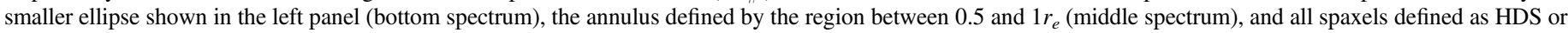

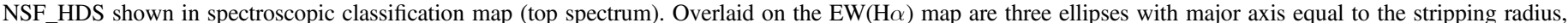

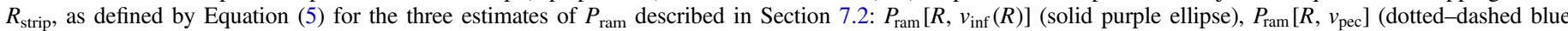

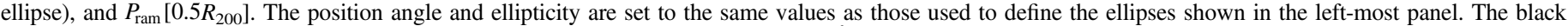

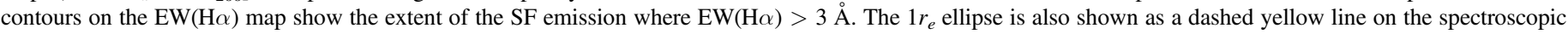
classification map.

GAMA HDSG and SFG $n_{\text {ser }}$ distributions, the KS test returns a probability $P=0.006$, rejecting the null hypothesis that they are drawn from the same parent population. Directly comparing the $n_{\text {ser }}$ distributions of the cluster and GAMA HDSGs, the KS test returns $P=0.012$, indicating that the two distributions are unlikely to be drawn from the same parent distribution. The differences in the $n_{\text {ser }}$ distributions suggest that the GAMA HDSGs may harbor larger bulge-to-total fractions than their cluster counterparts.

The differences in the environments, spectral properties, and structure of the cluster and GAMA HDSGs indicate that the GAMA HDSGs are not being quenched in the same manner as the cluster HDSGs. While the GAMA HDSGs are an interesting subset of the HDSGs selected here, further detailed analysis of their properties is beyond the scope of this paper. We will instead analyze a larger sample of HDSGs drawn from the full SAMI-GS sample in a future paper. For the remainder of this paper, we will focus on investigating the environments and properties of the cluster HDSGs.

\subsection{Demographics of the Cluster HDSGs}

Having identified several significant differences between the cluster and GAMA HDSGs, we now focus on the cluster 

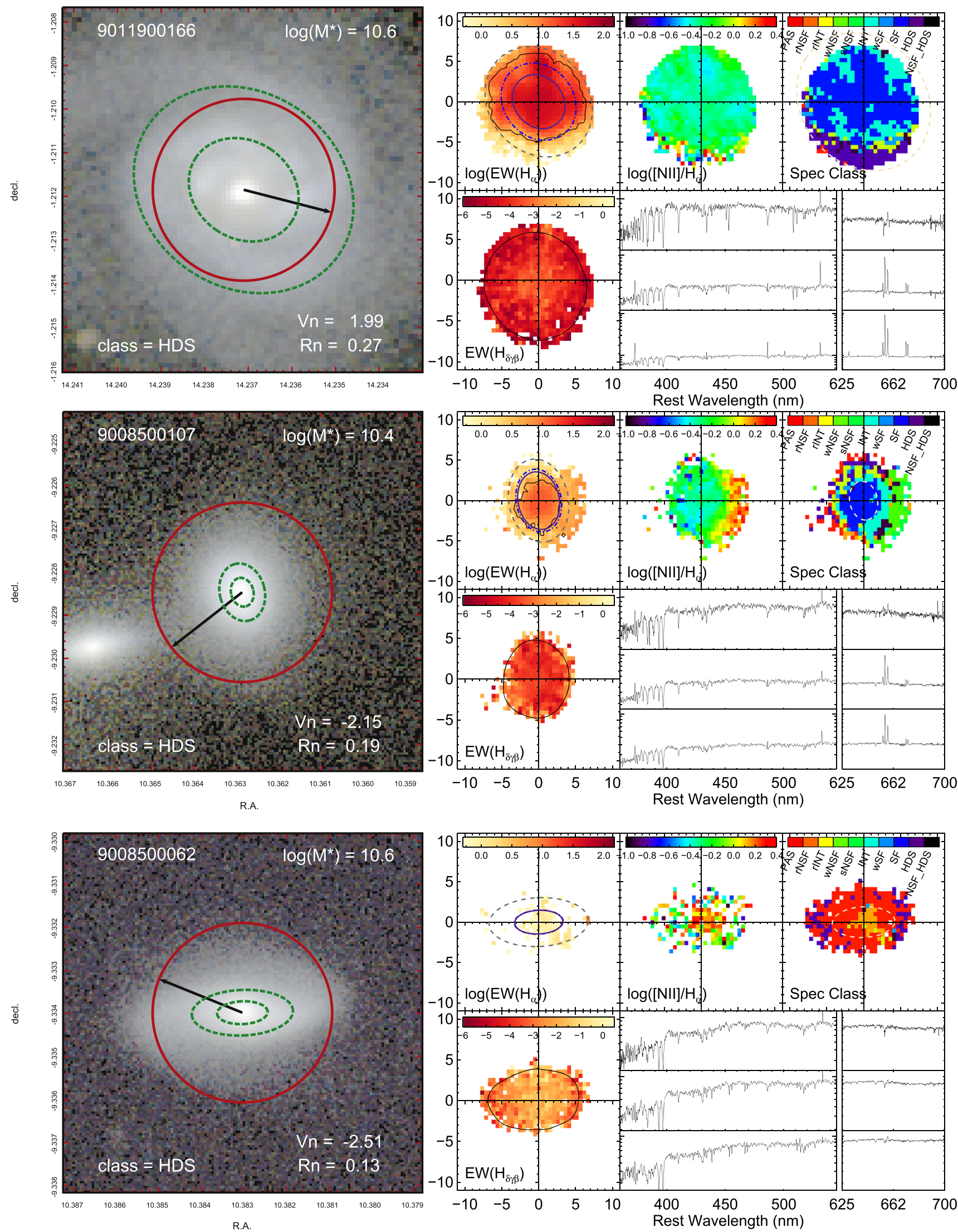

Figure 7. (Continued.) 

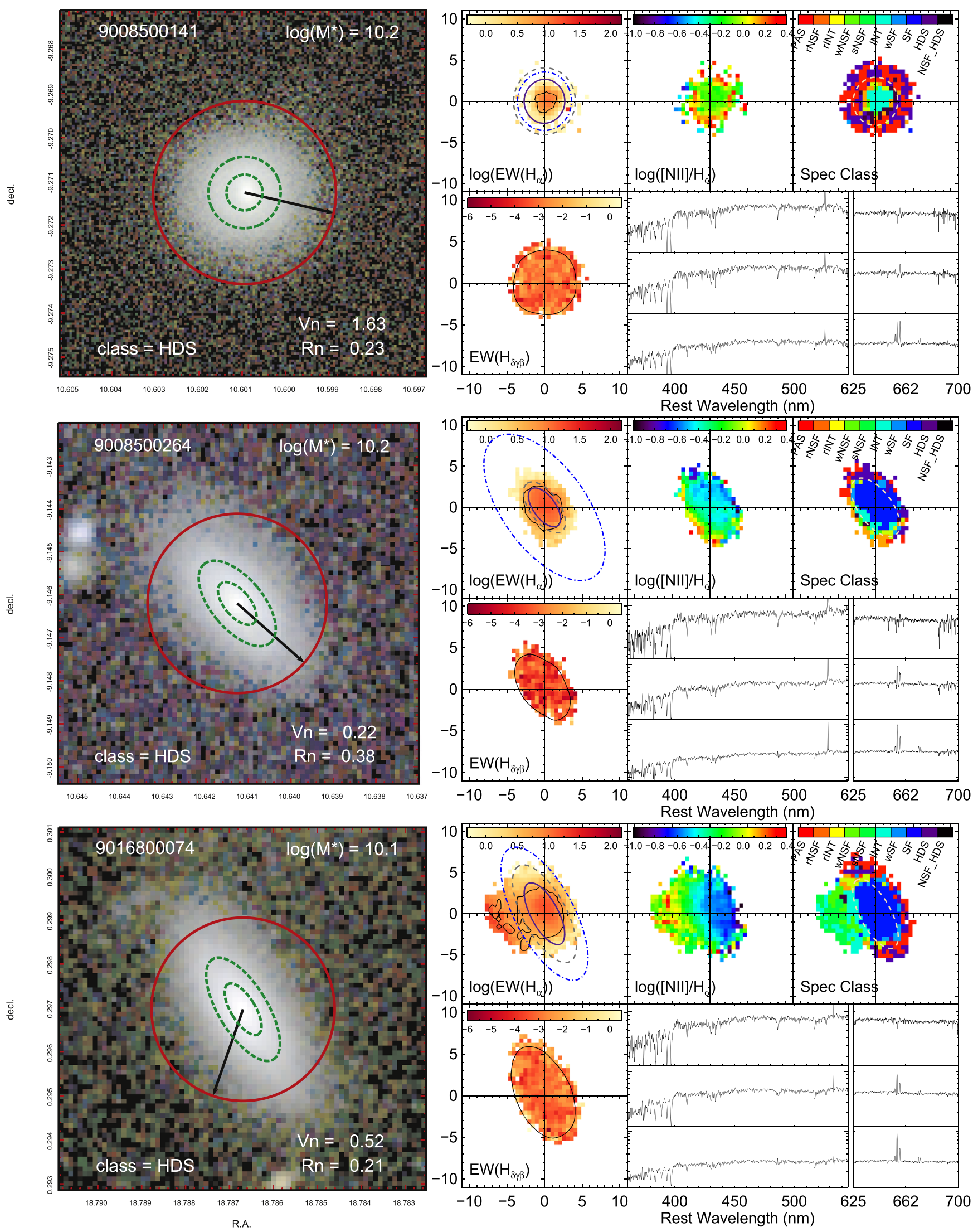

Figure 7. (Continued.) 

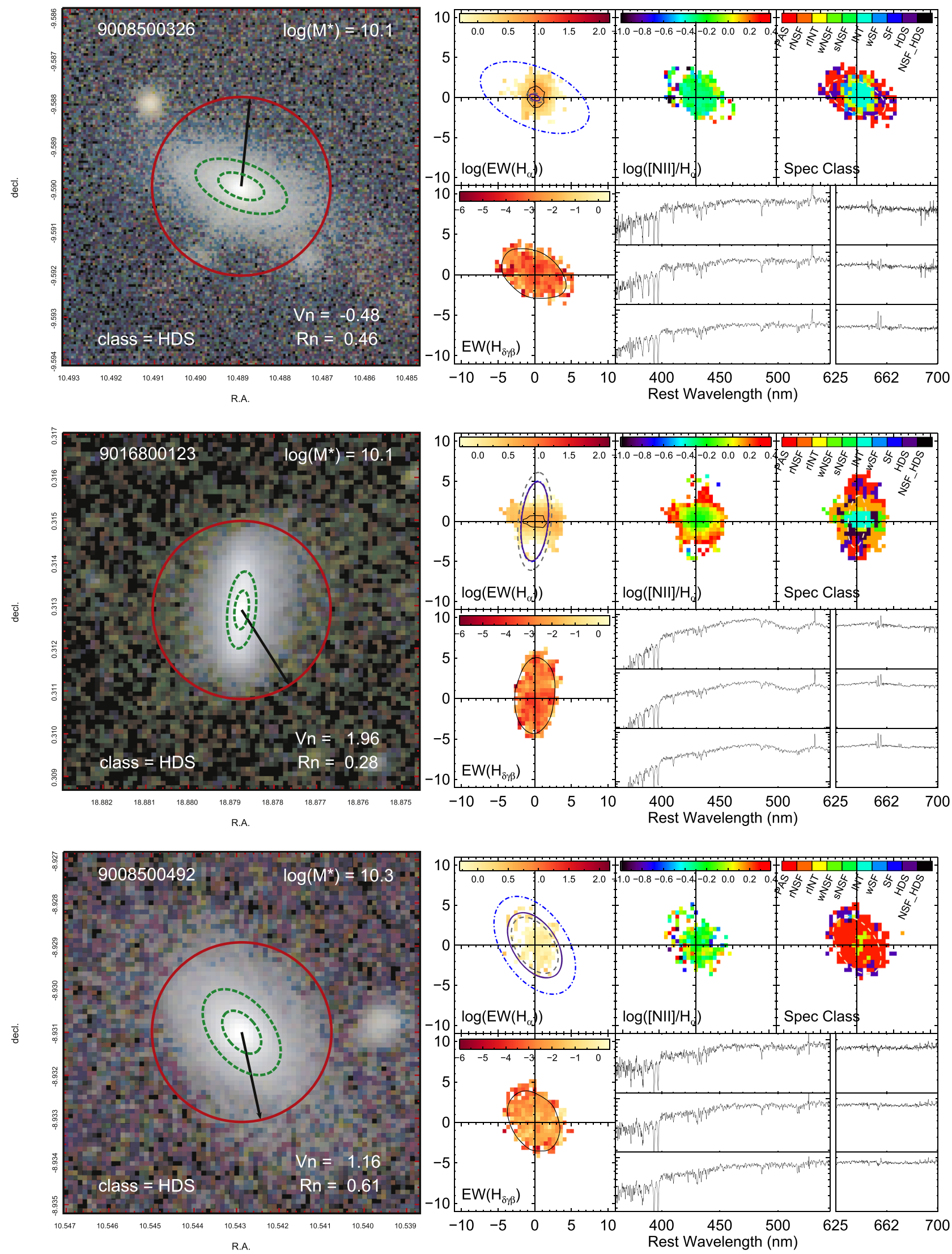

Figure 7. (Continued.) 

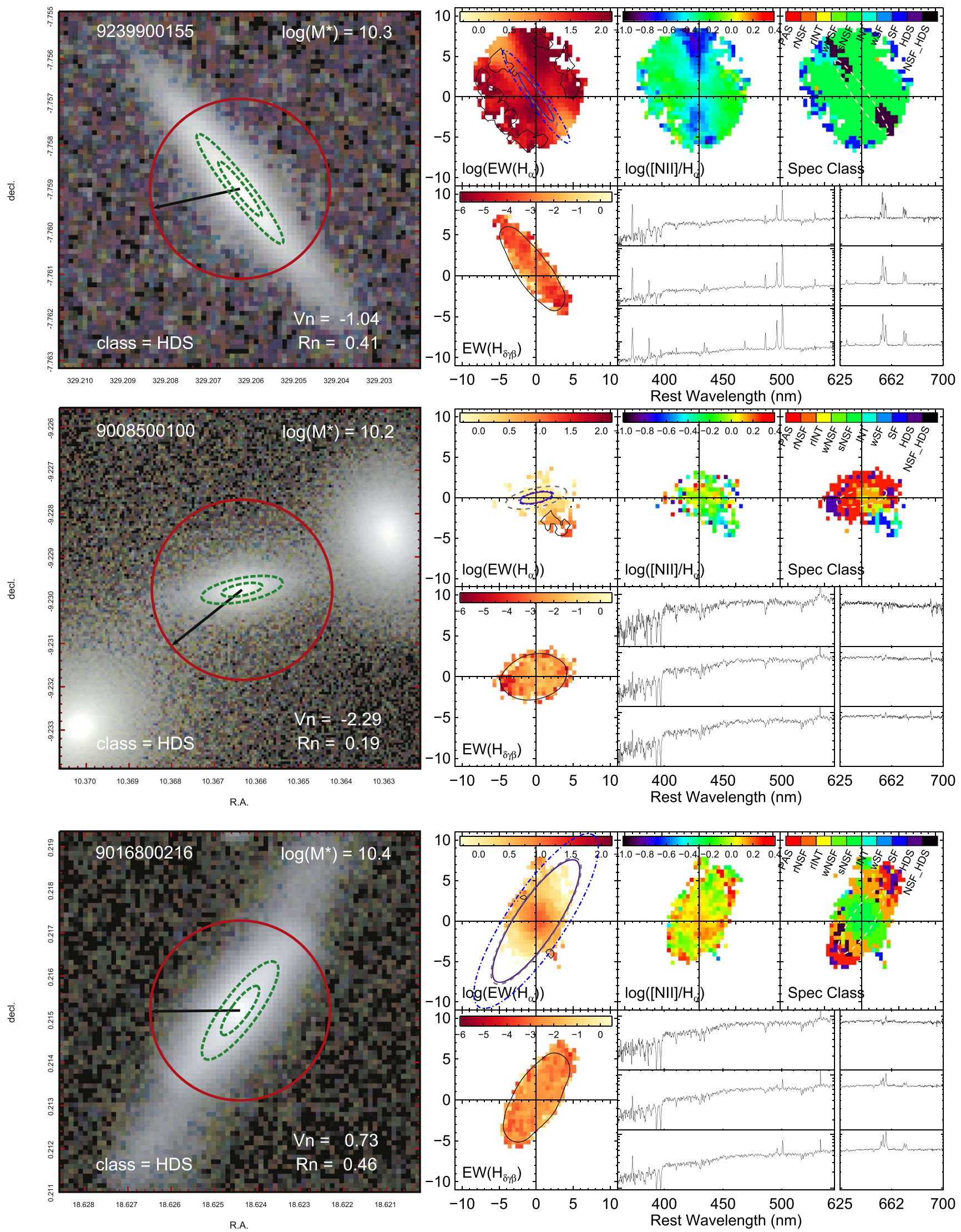

Figure 7. (Continued.) 

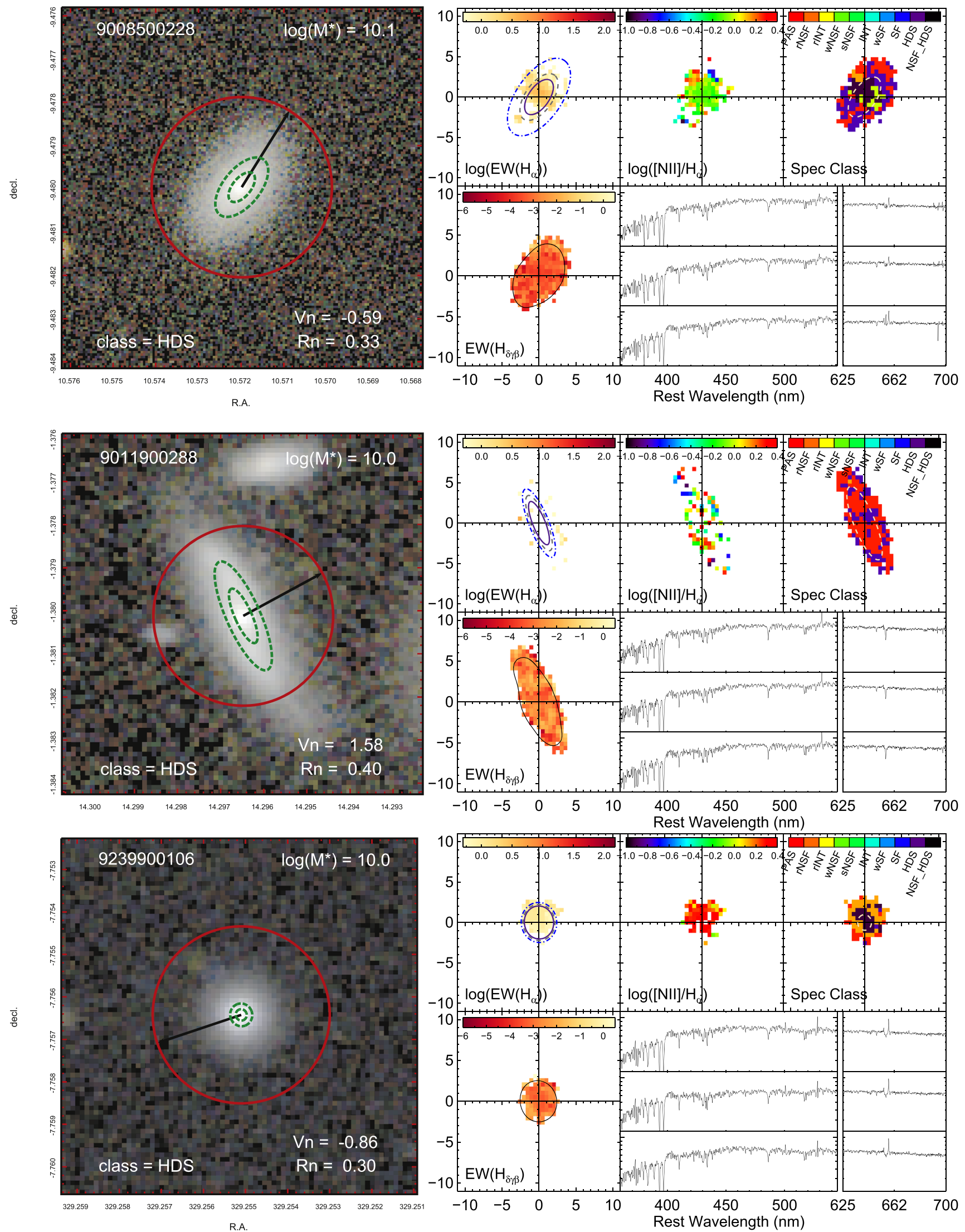

Figure 7. (Continued.) 

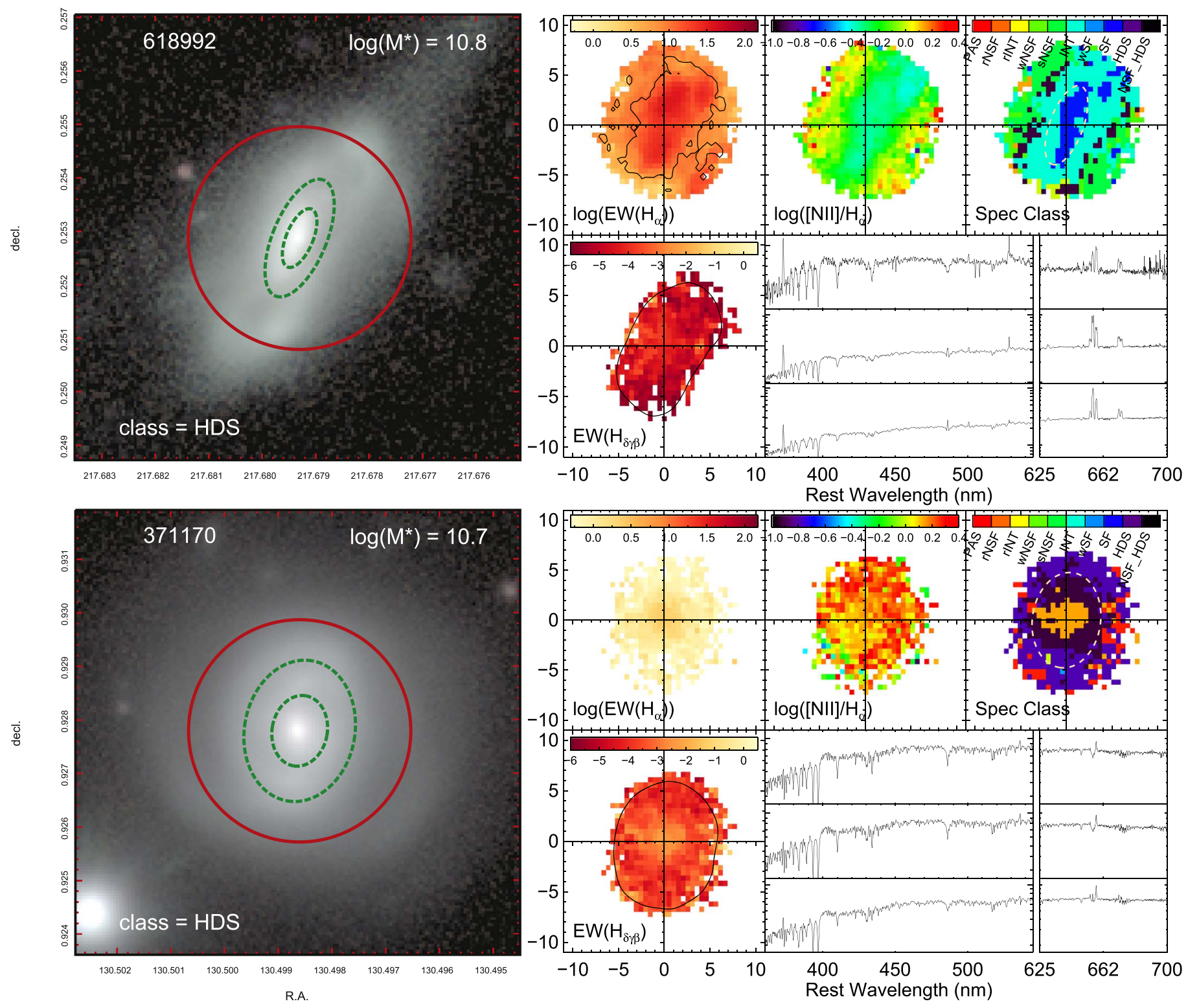

Figure 8. Same as Figure 7, but for the two most massive HDSGs selected from the GAMA portion of the survey.

regions. Our aim here is to identify correlations with clusterspecific environment metrics in order to understand which, if any, environment-related quenching processes may be at play. In Sections 6.2.2-6.2.4 we investigate the variations in the radial, velocity, and projected phase space (PPS) distributions for the PASGs, SFGs, and HDSGs. Because the HDSG sample is relatively small, we produce an ensemble cluster by stacking the normalized coordinates $R / R_{200}$ and $v_{\text {pec }} / \sigma_{200}$ across the eight SAMI-GS clusters.

\subsubsection{Star-forming Properties}

We noted in Section 6.1 that many of the cluster HDSGs show evidence of ongoing star formation at their centers, implying that the star formation in the cluster HDSGs is being quenched in an outside-in fashion. In Figure 10, we quantify this outside-in quenching by showing the distribution of the concentration of $\mathrm{H} \alpha$ flux relative to the continuum, $C_{\mathrm{H} \alpha \text {, cont }}$, for the cluster HDSGs (green histogram) with central star formation, along with the cluster SFGs (blue histogram). The $C_{\mathrm{H} \alpha \text {,cont }}$ values are determined in a fashion similar to that described in Schaefer et al. (2017). Briefly, we measure the cumulative flux profile in elliptical apertures centered on each galaxy using the ellipticity and PA determined by the Sérsic fits to the $r$-band data described in Section 2.2.1. For both the $\mathrm{H} \alpha$ and continuum flux (where the continuum flux level is determined in emission-free bands surrounding the $\mathrm{H}_{\alpha}$ line), the radius containing $50 \%$ of the flux, $r_{50, H \alpha}$ and $r_{50, \text { cont }}$, respectively, is measured and the concentration is determined as $C_{\mathrm{H} \alpha \text {,cont }}=r_{50, \mathrm{H} \alpha} / r_{50, \text { cont }}$. Note that in determining cumulative flux used to measure $r_{50, \mathrm{H} \alpha}$, only spaxels that are classified as INT, SF, or wSF are included. Thus, $\mathrm{H}_{\alpha}$ flux that is due to NSF ionization processes is not included in the $C_{\mathrm{H} \alpha}$ measurement. Figure 10 shows that $C_{\mathrm{H} \alpha \text {,cont }}<1$ for all HDSGs with central star formation, and that their $C_{\mathrm{H} \alpha \text {,cont }}$ values are much lower when compared with the majority of the SFGs. A KS test returns $P \ll 0.001$, thereby rejecting the hypothesis that the HDSG and SFG $C_{\mathrm{H} \alpha \text {,cont }}$ distributions are drawn from the same 

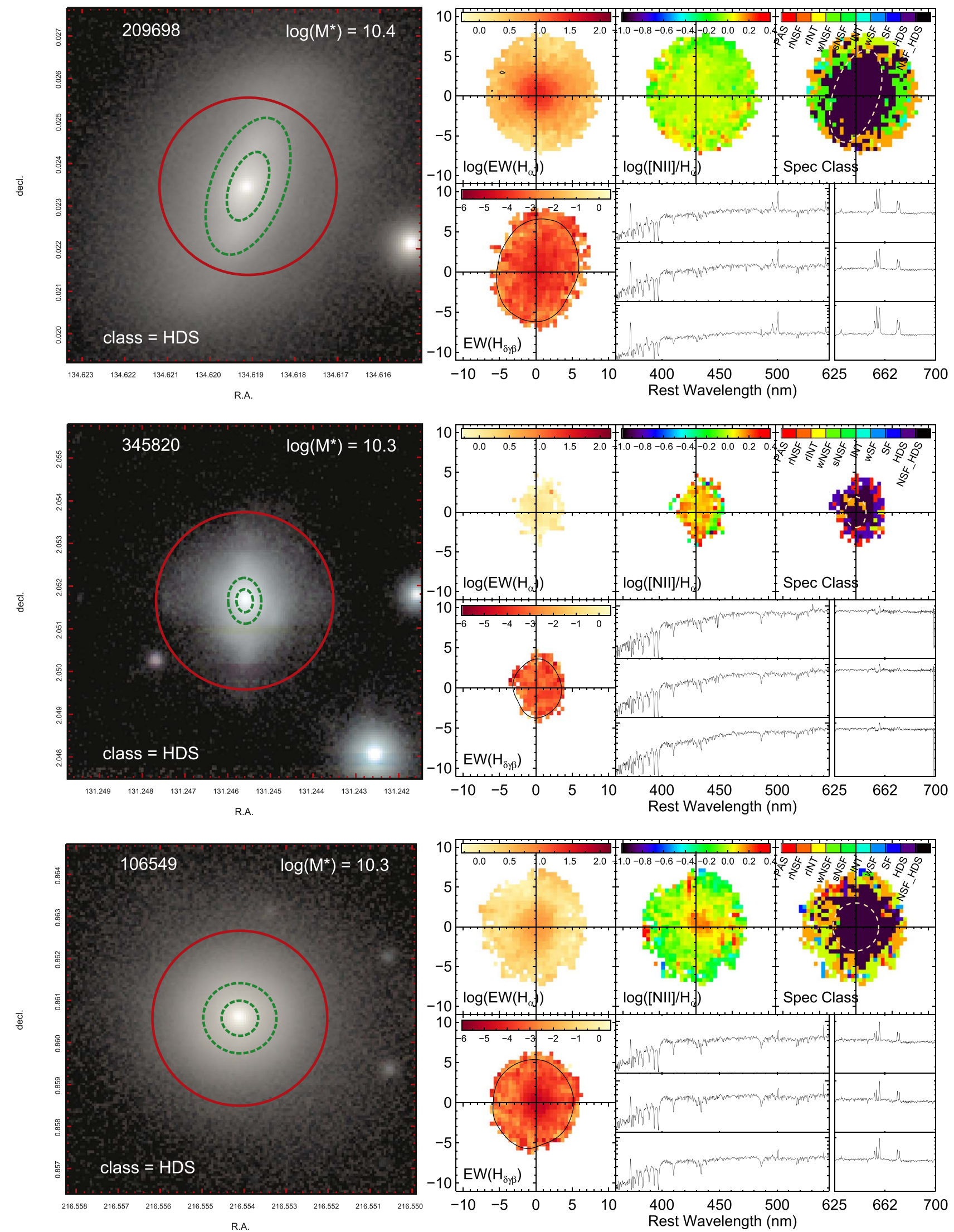

Figure 8. (Continued.) 

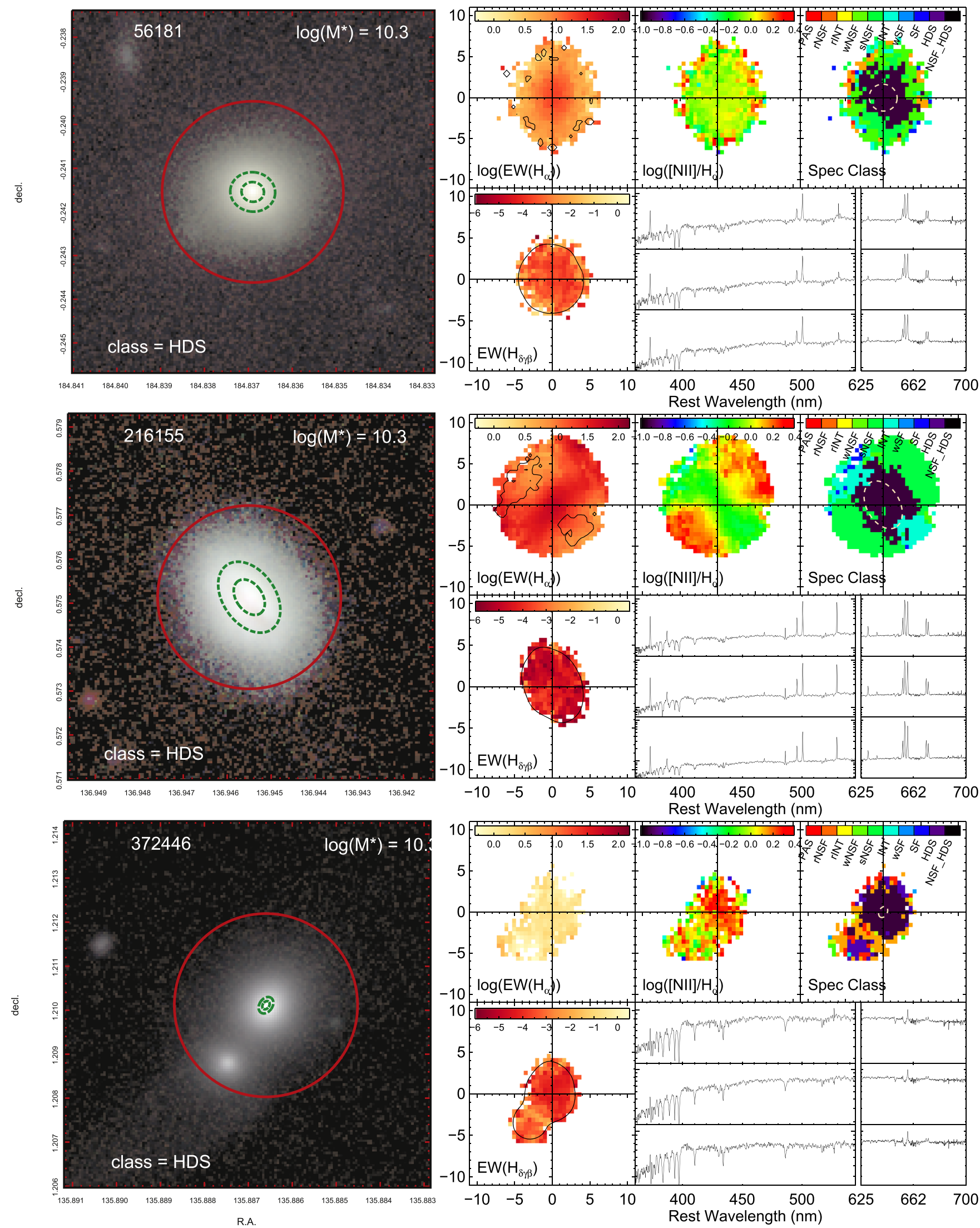

Figure 8. (Continued.) 

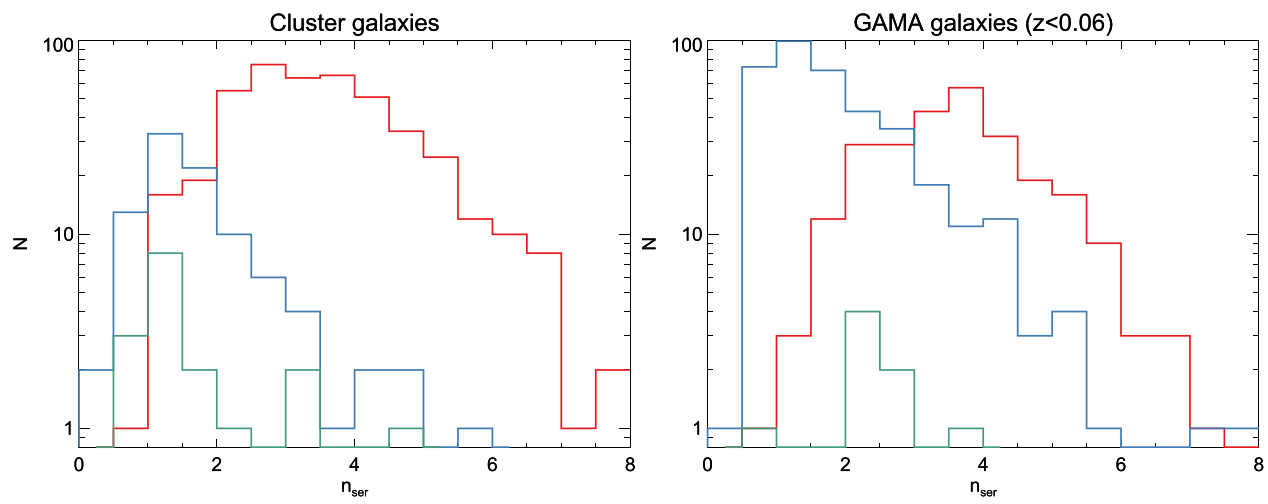

Figure 9. Distribution of Sérsic index $n_{\text {ser }}$ for SAMI galaxies in the clusters (left panel) and GAMA regions (right panel) separated by galaxy class where the red, blue, and green histograms show the $n_{\text {ser }}$ distribution of the PASG, SFG, and HDSG samples, respectively. The majority of the cluster HDSGs have $n_{\text {ser }}<2$, and their distribution is statistically consistent with the $n_{\text {ser }}$ distribution of the cluster SGFs. The majority of the GAMA HDSGs have $n_{\text {ser }}>2$, and the KS test indicates that the GAMA HDSG and SFG $n_{\text {ser }}$ distributions are not drawn from the same parent population.

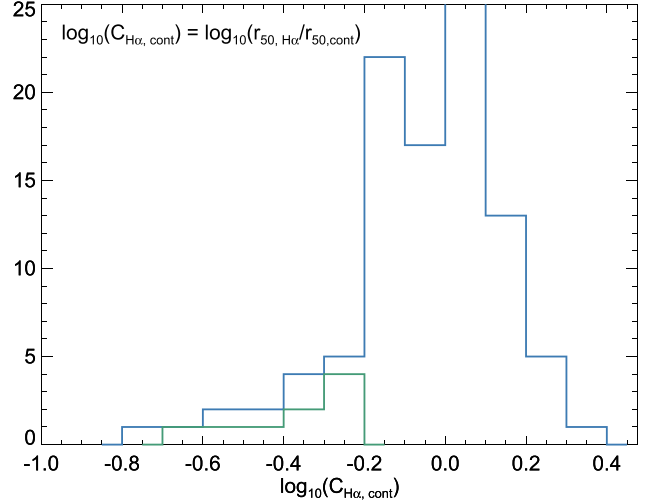

Figure 10. Concentration of $\mathrm{H}_{\alpha}$ flux relative to continuum flux, $C_{\mathrm{H} \alpha \text {, cont }}$ for the cluster HDSGs with central star formation (green histogram). Also shown is the distribution for the cluster SFGs. The star formation in the HDSGs is much more concentrated when compared with the SFGs, indicating that the cluster HDSGs are being quenched from the outside-in.

parent distribution. We note that while the majority $(68 \%)$ of the cluster SFGs show evidence of ongoing star formation at their centers, a substantial fraction do not. We therefore repeated the comparison between the HDSG and SFG $C_{\mathrm{H}} \alpha$, cont distributions including only those SFGs with central star formation, finding that our main result remains unchanged.

Many of the environmental processes introduced in Section 1 predict enhanced star formation at the centers of affected galaxies, which may, in turn, lead to the more concentrated $\mathrm{H} \alpha$ flux revealed for the HDSGs in Figure 10. We test for evidence of central starbursts in Figure 11 where we show the distribution of the median $\mathrm{EW}(\mathrm{H} \alpha)$ of the spaxels within $0.5 r_{e}$ for each of the HDSGs with central star formation, as well as the cluster SFGs. Again, only spaxels that are classified as SF, wSF, or INT are used in determining the median $\mathrm{EW}(\mathrm{H} \alpha)$. We find no significant difference when comparing the $\mathrm{EW}(\mathrm{H} \alpha)$ distribution for the HDSGs and SFGs; a KS test does not reject the hypothesis that the two distributions are drawn from the same parent population, returning a probability $P=0.68$. This similarity in the $\mathrm{EW}(\mathrm{H} \alpha)$ distributions indicates that, while the spatial distribution of star formation in a large portion of the HDSGs is more concentrated than that seen in the cluster SFGs, the mode of star formation does not appear to be dramatically different.

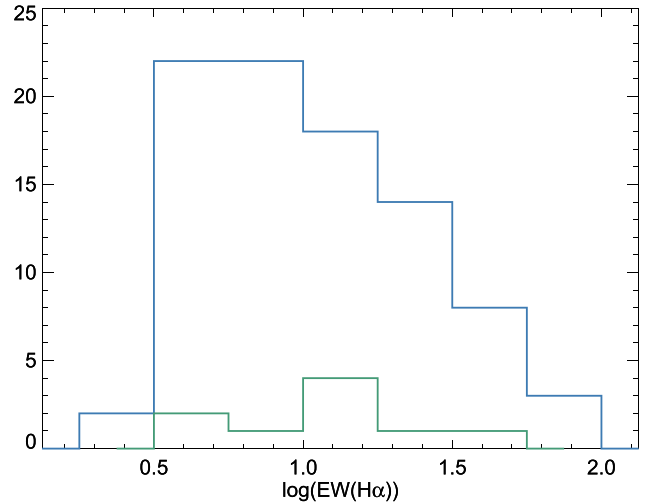

Figure 11. Distribution of the median emission line $\mathrm{EW}(\mathrm{H} \alpha)$ measured within $0.5 r_{e}$ for the cluster HDSGs with central star formation (green histogram) and the cluster SFGs (blue histogram). The distributions do not differ significantly, indicating that the mode of star formation in the centers of the HDSGs and SFGs is not different.

\subsubsection{Projected Cluster-centric Distance}

It is well established that the fraction of cluster PASGs increases with decreasing cluster-centric distance, while the fraction of SFGs increases with increasing cluster-centric distance (Lewis et al. 2002; von der Linden et al. 2010; Haines et al. 2015; Barsanti et al. 2018). More recently, Paccagnella et al. (2017) have found that the fraction of HDSGs increases by a factor of $\sim 1.7$ going from the outskirts to the center of low-redshift clusters, although their selection is based on single-fiber spectroscopy. The left and right panels of Figure 12 show, respectively, the distribution and fractions of the PASGs, SFGs, and HDSGs (red, blue, and green lines, respectively) as a function of normalized cluster-centric distance. The normalized projected cluster-centric distances, $R / R_{200}$, are measured from the cluster centers listed in Table 1 of Owers et al. (2017). The corresponding $68 \%$ confidence intervals shown in the right panel of Figure 12 were calculated per the method described by Cameron (2011). The fractions shown as histograms in Figure 12 are not corrected for the radial- and stellar-massdependent incompleteness of the sample. The completenesscorrected fractions are shown as open circles and are calculated by determining a weighting for each galaxy in the sample that accounts for the radius- and stellar-mass-dependent completeness. The corrected fractions do not differ significantly from the uncorrected values. 

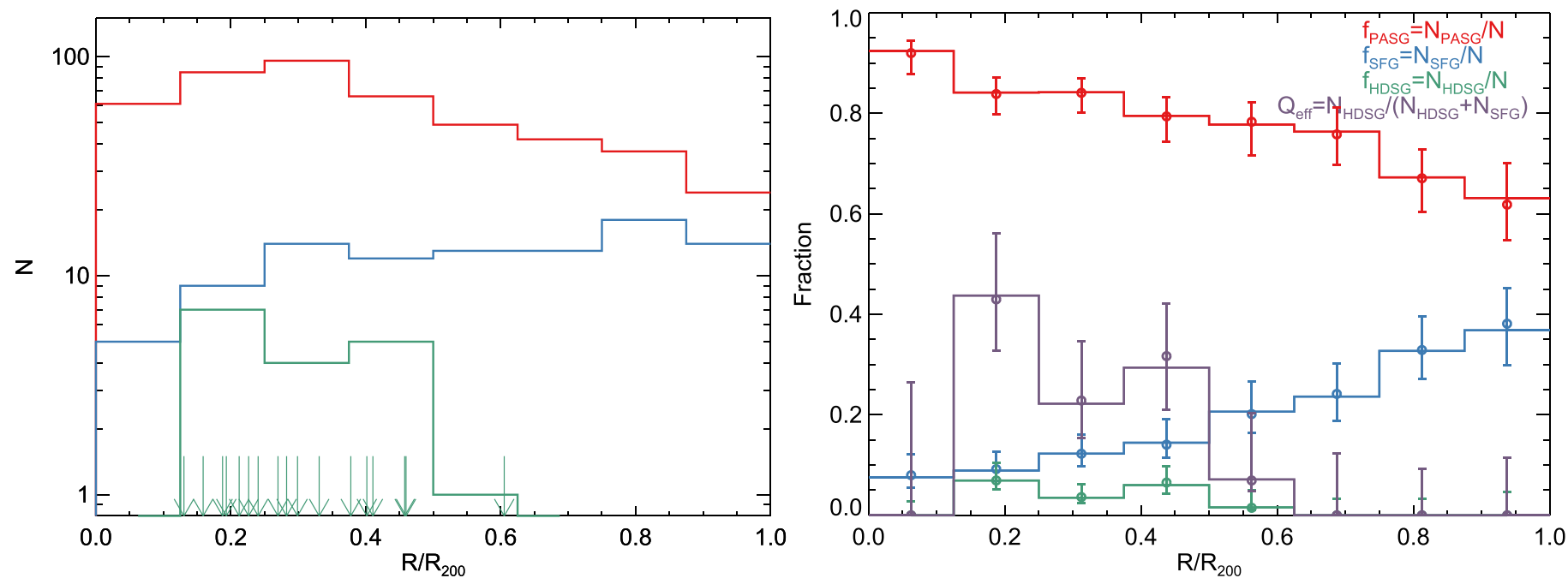

Figure 12. The left panel shows the binned distribution of cluster galaxies with different spectral types as a function of $R / R_{200}$. The positions of the HDSGs are indicated by green arrows. The histograms in the right panel shows the corresponding binned fractions for each of the PASG, SFG, and HDSG samples. For both panels, the PASGs, SFGs, and HDSGs are shown as red, blue, and green lines, respectively. In the right panel, the circles show the completeness-corrected fractions, which do not differ significantly from the noncorrected fractions (shown as histograms). The purple line in the right panel shows the quenching efficiency, $Q_{\text {eff }}$, defined as the fraction of HDSGs relative to the total (SFGs+HDSGs) active galaxies. The HDSGs all have $R / R_{200}<0.6$, and are more concentrated toward the cluster centers than the cluster SFG distribution.

We find that the vast majority (16/17) of the HDSGs are within the radial range $0.125<R / R_{200}<0.5$, where $f_{\mathrm{HDSG}} \sim 5 \%$; only galaxy 9008500492 has $R / R_{200}=0.6$. Inspection of the spectrum derived by stacking the HDS regions shown in Figure 7 indicates that 9008500492 has only relatively weak Balmer absorption and is the least convincing HDSG in our sample; only 23 of 199 spaxels are classified as HDS or NSF_HDS. In Section 6.1 we found that $Q_{\text {eff }}=2.0 \%$ for the GAMA portion of the survey, which is assumed to be representative of the field population that is accreted onto clusters. Given this, we may expect one or two SFGs that are falling into the clusters to be undergoing similar quenching to that observed in the GAMA HDSGs. This may explain the relatively large projected cluster-centric distance of 9008500492.

The small number of HDSGs found outside $0.5 R_{200}$ indicates that the true 3D location of the HDSGs is also within $0.5 r_{200,3 D}$, and not due to projection effects. The left panel of Figure 12 shows that the number of PASGs and SFGs increases with radius to $R / R_{200}=0.3$, but shows differing behavior thereafter, with the number of PASGs declining with radius and the number of SFGs remaining relatively flat with increasing radius. Both the two-sample KS and Anderson-Darling (AD) tests return a probability $P \ll 0.001$, strongly rejecting the hypothesis that the differences in the cumulative distribution functions of the radii of the PASGs and SFGs can occur by random chance if the two samples were drawn from the same parent distribution. Similarly, both the AD and KS tests return $P \ll 0.001$ for the comparison between the HDSG and SFG radial distributions. The comparison between the HDSG and PASG returns $P=0.06$ and $P=0.08$ for the KS and AD tests, respectively, indicating that we cannot reject the hypothesis that the two radial distributions are drawn from the same parent population. We note that repeating the comparisons between the PASG and HDSG $R / R_{200}$ distributions after removing galaxy 9008500492 from the HDSG sample returns $P=0.019$ and $P=0.043$ for the KS and AD tests, respectively. These tests confirm what can be deduced by inspection of the left panel of Figure 12: the HDSG sample is significantly more

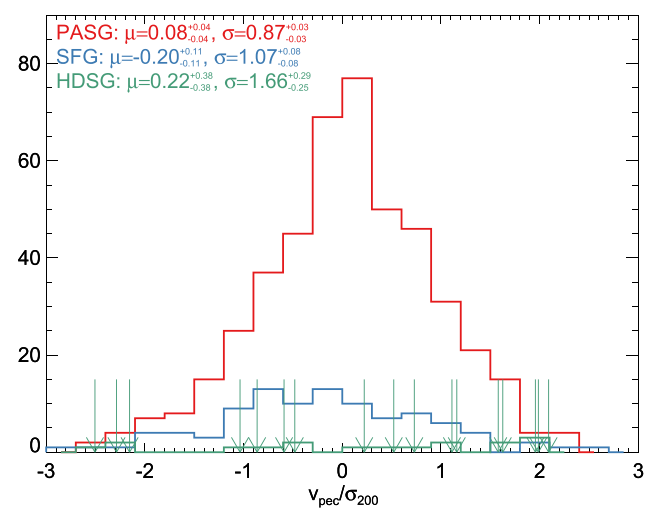

Figure 13. $v_{\mathrm{pec}} / \sigma_{200}$ distribution of the PASGs (red), SFGs (blue), and HDSGs (green). The locations of the HDSGs are highlighted by green arrows. The biweight estimates for the mean, $\mu$, and standard deviation, $\sigma$, are shown for each sample at the top left of the panel.

concentrated toward the cluster centers than the SFG sample and does not appear to follow the same radial distribution as the PASG sample, although the latter result is not as statistically robust as the former.

Figure 12 also shows that the quenching efficiency, $Q_{\text {eff }}$ (purple line; defined as in Section 6.1), is largest in the $R / R_{200} \simeq 0.2$ bin, where it is $44 \%$, and decreases to $\sim 20 \%-$ $30 \%$ in the next two larger radial bins. However, we note that the $68 \%$ confidence intervals overlap between the bins with $R / R_{200}<0.5$, so the increase is not statistically significant. Both the PASG and SFG fractions follow the expected radial trends, with the PASG fraction declining from $f_{\mathrm{PASG}}=92 \%$ at $R / R_{200}<0.125$ to $f_{\mathrm{PASG}}=63 \%$ in the $R / R_{200}=0.875-1 \mathrm{bin}$, and the SFG fraction increasing from $f_{\mathrm{SFG}}=8 \%$ at $R / R_{200}<0.125$ to $f_{\mathrm{SFG}}=37 \%$ in the $R / R_{200}=0.875-1$ bin.

\subsubsection{Velocity Distribution}

Figure 13 shows the relative line-of-sight (LOS) velocity distribution, $v_{\text {pec }} / \sigma_{200}$, for the PASG (red), SFG (blue), and 


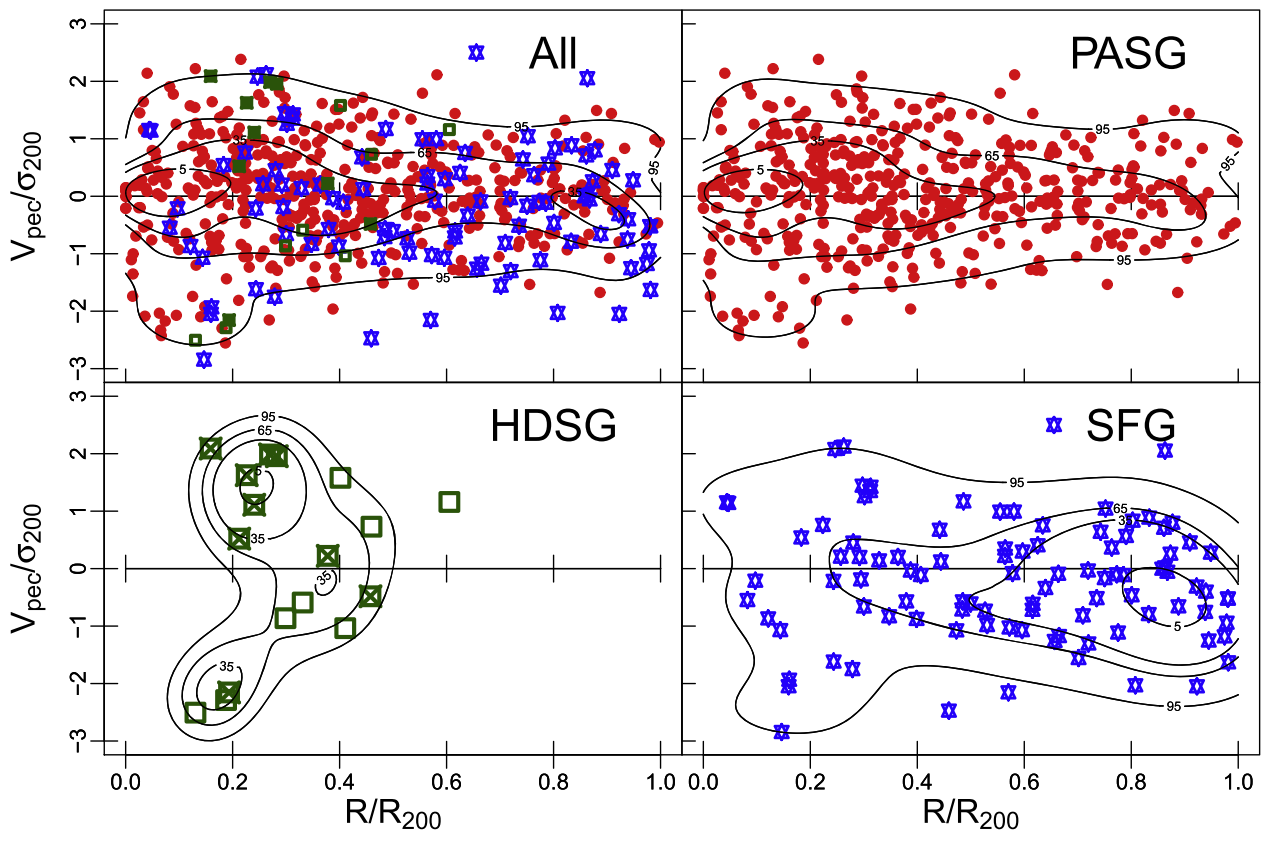

Figure 14. PPS distribution of SAMI-GS cluster galaxies. The red circles, blue stars and green squares show the positions of PASGs, SFGs, and HDSGs, respectively. HDSGs with central star formation are highlighted with green crosses. The black contours show the smoothed density distribution corresponding to the sample named in the upper right of each plot. The HDSGs occupy a coherent, arc-shaped region in the PPS that is very different from the distribution of SFGs.

HDSG (green) samples. In the upper left of Figure 13, for each sample we list the mean, $\mu$, and standard deviation, $\sigma$, determined using biweight estimators (Beers et al. 1990), and their associated uncertainties, which are determined using jackknife resampling. The distributions do not appear to depart significantly from a Gaussian shape; both the KS and AD tests fail to reject the hypothesis that any of the PASG, SFG, or HDSG distributions are drawn from a Gaussian parent distributions.

Velocity segregation is readily observed between the passive and SF cluster populations, regardless of the proxy used to distinguish the two populations (e.g., morphology, color, or the presence/absence of emission lines; Colless \& Dunn 1996; Biviano et al. 1997; Barsanti et al. 2016). This velocity segregation is attributed to the different dynamical states of the PASGs and SFGs. The PASGs form the bulk of the virialized cluster population, whereas the SF spirals are the dominant population of infalling galaxies and follow more radial orbits (Biviano \& Katgert 2004). We also find notable differences when comparing the PASG with both the SFG and HDSG $v_{\text {pec }} / \sigma_{200}$ distributions. The $\sigma$ values measured for the SFG and HDSG samples indicate that they have larger dispersions than the PASGs. This is particularly true for the HDSGs compared with the PASGs, where the measured uncertainties indicate that the difference in $\sigma$ is significant with $99.9 \%$ confidence, while the SFG $\sigma$ is larger than that of the PASG with $>95 \%$ confidence. The measured $\mu$ for the HDSG and SFG samples are offset from the PASG value, although the uncertainties indicate that the differences are not statistically significant.

We note that the dispersion of the PASGs is $\sigma=0.87_{-0.03}^{+0.03} \sigma_{200}$, which implies that their dispersion is significantly lower than that expected of a virialized population. This lower dispersion is likely due to the different samples used to define the $\sigma_{200}$ values determined by Owers et al. (2017), which used all spectroscopically confirmed cluster members within $R_{200}$, and therefore includes a contribution from a larger fraction of less-massive SFGs. Their inclusion leads to an overall higher estimate of $\sigma_{200}$ and, therefore, lower normalized velocities; indeed, the dispersion measure using all SAMI-GS cluster galaxies is $\sigma=0.94_{-0.03}^{+0.03}$.

Comparing the samples against each other using the twosample KS test, the hypothesis that the PASG and SFG $v_{\text {pec }} / \sigma_{200}$ distributions are drawn from the same parent distribution is rejected $(P<0.01)$, but the $\mathrm{KS}$ test fails to reject this hypothesis for the HDSG versus SFG and HDSG versus PASG comparisons. The $\mathrm{KS}$ test is most sensitive to differences that occur around the centers of the distributions, whereas the AD test has better sensitivity to departures in the tails of two distributions. Using the two-sample AD test, we find that we can reject the hypothesis that the PASG, HDSG, and SFG $v_{\text {pec }} / \sigma_{200}$ distributions are drawn from the same parent distribution $(P=0.009, P=0.04$, and $P=0.005$ for the HDSG versus PASG, HDSG versus SFG, and SFG versus PASG, respectively). Taking the results of the AD tests in concert with their different $\sigma$ values, it is likely that the SFG, HDSG, and PASG samples are kinematically distinct from each other.

\subsubsection{PPS Distribution}

In Sections 6.2.2 and 6.2.3, we showed that there are differences in both the radial and velocity distributions of the PASG, HDSG, and SFG samples. While these differences in radial and velocity distributions are informative when considered separately, the combination of LOS velocity and radius can be a more powerful discriminant of populations with different accretion histories. Simulations of clusters reveal that infalling and recently accreted galaxies occupy PPS regions that are relatively distinct from the virialized population even when knowledge of the full 6D phase space information is confused by projection effects (Gill et al. 2005; Oman et al. 2013; Haines et al. 2015; Rhee et al. 2017). Therefore, the PPS is recognized as a powerful diagnostic for understanding quenching in clusters (Biviano et al. 1997; Solanes et al. 2001; 
Table 3

Fraction of Spectral Types in Various PPS Regions

\begin{tabular}{|c|c|c|c|c|c|c|c|}
\hline$R / R_{200}$ & $\left|V_{\mathrm{pec}}\right| / \sigma_{200}$ & $\mathrm{~N}_{\text {SAMI }}$ & Completeness & $f_{\mathrm{PASG}}$ & $f_{\mathrm{SFG}}$ & $f_{\text {HDSG }}$ & $Q_{\text {eff }}$ \\
\hline $0.0-0.5$ & $0.0-1.5$ & 309 & $89_{-2}^{+1}$ & $87_{-2}^{+2}$ & $10_{-1}^{+2}$ & $3_{-1}^{+1}$ & $20_{-5}^{+8}$ \\
\hline $0.0-0.5$ & $1.5-3.0$ & 55 & $81_{-6}^{+4}$ & $71_{-7}^{+5}$ & $15_{-4}^{+6}$ & $15_{-4}^{+6}$ & $50_{-12}^{+12}$ \\
\hline $0.5-1.0$ & $0.0-1.5$ & 199 & $83_{-3}^{+2}$ & $74_{-3}^{+3}$ & $26_{-3}^{+3}$ & $1_{-0}^{+1}$ & $2_{-1}^{+4}$ \\
\hline $0.5-1.0$ & $1.5-3.0$ & 12 & $75_{-13}^{+8}$ & $42_{-12}^{+14}$ & $58_{-14}^{+12}$ & $0_{-0}^{+13}$ & $0_{-0}^{+21}$ \\
\hline
\end{tabular}

Vollmer et al. 2001; Mahajan et al. 2011; Noble et al. 2013; Muzzin et al. 2014; Jaffé et al. 2015; Oman \& Hudson 2016; Barsanti et al. 2018).

In the top left panel of Figure 14, we show the stacked PPS for SAMI galaxies in the eight clusters. The PASGs, SFGs, and HDSGs are plotted as filled red circles, blue stars, and green squares, respectively. The top right, bottom left, and bottom right panels in Figure 14 show, separately, the PPS distributions for the PASGs, HDSGs, and SFGs, respectively. Those HDSGs that show evidence for star formation at their centers are highlighted by green crosses. In each of the four panels, contours are generated from the smoothed kernel density estimate (KDE) for the distribution of points, which is determined using the ks software ${ }^{28}$ (Duong 2007).

Figure 14 shows that the distribution of HDSGs at small radius is strikingly different from both the PASGs and SFGs. The distribution of HDSGs forms an arc-like shape in PPS, with the smallest projected radius $\left(R / R_{200} \lesssim 0.3\right)$ having large velocities $\left|v_{\text {pec }}\right| / \sigma_{200}>1.0$, whereas those with $R / R_{200} \gtrsim 0.3$ have lower velocities $\left|v_{\text {pec }}\right| / \sigma_{200}<1.0$. The HDSGs are absent from the low velocity and small radius $\left(R / R_{200}<0.2\right)$ part of the PPS, which is dominated by PASGs. Interestingly, the HDSGs that do not have evidence for star formation at their centers primarily occupy the small velocity, large radius region of the arc-like shape in PPS. The KDE contours reveal that the PASGs and SFGs exhibit a mirror-flipped distribution about the $R / R_{200}$ axis: the density of SFGs increases with radius and the velocity spread stays relatively constant, whereas the density and velocity spread of PASGs decrease with radius.

We test the significance of the difference observed in the PPS distributions for the PASG, SFG, and HDSG samples using the multivariate two-sample $\mathrm{KDE}$ test developed by Duong et al. (2012) for the purpose of comparing cell morphologies and recently applied to PPS distributions by Lopes et al. (2017) and de Carvalho et al. (2017). The KDE test is nonparametric and uses the integrated square error as a measure of the discrepancy between two KDEs to test the hypothesis that two distributions are drawn from the same underlying density distribution (see Duong et al. 2012, for details). The test returns $P \ll 0.01$ for both of the PASG versus SFG and SFG versus HDSG comparisons, rejecting the hypothesis that these distributions are drawn from the same underlying density distribution. Similarly, a two-sample 2D KS test (Peacock 1983) returns $P \ll 0.001$ for these two comparisons. The comparison of the PASG and HDSG distributions yields less significant results, with the KDE test returning $P=0.02$ and the $2 \mathrm{D} \mathrm{KS}$ test returning $P=0.12$. Removing galaxy 9008500492 from the sample and rerunning the $\mathrm{KDE}$ and $\mathrm{KS}$ tests return $P=0.01$ and $P=0.08$, respectively. We also use the $2 \mathrm{D} \mathrm{KS}$ test to compare the three PPS distributions using the absolute value of the velocity,

\footnotetext{
${ }^{28}$ https://cran.r-project.org/web/packages/ks/index.html
}

$\left|v_{\text {pec }}\right| / \sigma_{200}$. For each of the three comparisons, the $2 \mathrm{D} \mathrm{KS}$ test returns $P \leqslant 0.001$. Therefore, we can confidently conclude that the SFG distribution is significantly distinct from both the PASG and HDSG distributions, while the HDSG and PASG distributions appear distinct, but with lower significance.

In Table 3, we show the fractions for the different galaxy types as a function of position in PPS. The PPS distributions are relatively symmetric about the velocity axis, which means that the absolute velocity, $\left|v_{\text {pec }}\right| / \sigma_{200}$, can be used to compare the PPS region, thereby enhancing the number of objects in each region without losing information. We divide the PPS into quadrants with boundaries listed in Table 3. Eight of 17 HDSGs are found in the $\left|v_{\text {pec }}\right| / \sigma_{200}>1.5, R / R_{200}<0.5$ quadrant, and in this region the fractional contribution of the HDSGs to the total active (SFG+HDSG) galaxy samples is $15 \%$ and $50 \%$, respectively, which is significantly higher than elsewhere in the PPS. The differences in the PPS distributions for the HDSGs, PASGs, and SFGs indicates that the three populations are at different stages in their accretion histories.

\subsubsection{Dependence on Cluster Mass}

We note that the majority $(11 / 17)$ of the cluster HDSGs are found in the two most massive clusters in the sample, A119 $\left(\mathrm{N}_{\mathrm{HDSG}}=3\right)$ and $\mathrm{A} 85\left(\mathrm{~N}_{\mathrm{HDSG}}=8\right)$. This majority may be expected given that these two clusters account for a large fraction of the total number of galaxies in the sample (119 and 150 galaxies for $\mathrm{A} 119$ and A85, respectively). However, simulations predict that more massive clusters may be more efficient at quenching star formation (e.g., due to stronger ram pressure; Bekki 2014), and Paccagnella et al. (2017) found that the $\mathrm{H} \delta$-strong-to-active galaxy fraction increases with increasing X-ray luminosity of their WIde-Field Nearby Galaxycluster Survey (WINGS) clusters. Figure 15 shows the completeness-corrected fraction of the PASGs, SFGs, and HDSGs as a function of cluster mass (red circles, blue stars, and green squares, respectively). The cluster mass is determined from the caustics method (outlined in Owers et al. 2017), plotted for each of the eight SAMI clusters, along with the quenching efficiency (purple hexagons) determined as in Section 6.1. The solid lines with the same color-coding show the fractions measured in two bins that divide the cluster halos at $M_{200}=5 \times 10^{14} M_{\odot}$. The binned results suggest that the fraction of PASGs is higher in the $M_{200}>5 \times 10^{14} M_{\odot}$ bin, along with a commensurate decrease in the fraction of SFGs. Interestingly, the value of $Q_{\text {eff }}$ does increase going from the low-mass to high-mass bin, where $Q_{\text {eff }}=29_{-6}^{+7} \%$, compared with $Q_{\text {eff }}=8_{-2}^{+4} \%$ in the low-mass bin. This increase in $Q_{\text {eff }}$ with cluster mass suggests that the more massive clusters are more efficient at quenching galaxies, although a larger sample of clusters is required to rule out halo-to-halo variations and confirm this result. 


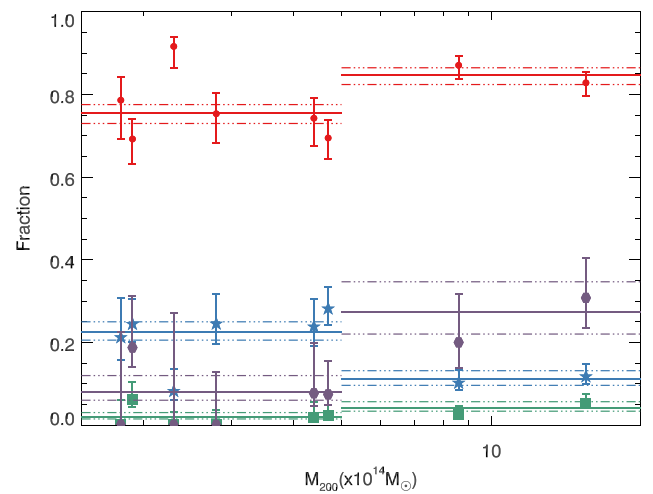

Figure 15. Fraction of PASGs, SFGs, and HDSGs as a function of cluster mass, shown for the individual clusters as red circles, blue stars, and green squares, respectively. The quenching efficiencies $\left(Q_{\text {eff }}=N_{\mathrm{HDSG}} /\left(N_{\mathrm{HDSG}}+N_{\mathrm{SFG}}\right)\right)$ for each cluster are shown as purple hexagons. The solid lines show the fractions of PASGs, SFGs, HDSGs, and $Q_{\text {eff }}$ in two mass bins for clusters with $14<\log \left(M_{*} / M_{\odot}\right)<14.7$ and $\log \left(M_{*} / M_{\odot}\right) \geqslant 14.7$ with the same color scheme as for the individual points. Dashed-dotted lines show the corresponding $68 \%$ confidence intervals. This suggests that clusters with $\log \left(M_{200} / M_{\odot}\right)>14.7$ have a higher $Q_{\text {eff }}$ when compared with lower mass clusters, although cluster-to-cluster variations may affect these results.

\section{Discussion}

The principal drivers of this study were to first identify galaxies that show evidence for ongoing quenching of star formation and then to characterize their environments in order to help understand which environmental quenching mechanisms are most germane. As a first step toward the latter goal, in Section 6.1 we compared the HDSGs found in the lower density GAMA regions with those found in the cluster regions. We found that the frequency of galaxies with HDS signatures was significantly higher among the nonpassive cluster population $\left(15_{-3}^{+4} \%\right)$ relative to that found in the GAMA regions $\left(2_{-0.4}^{+1.0} \%\right)$. This indicates that the cluster environment is more efficiently quenching the SFGs.

Furthermore, we found three notable differences between the GAMA and cluster HDSGs that indicate that the quenching mechanisms acting in the two samples are different. First, none of the GAMA HDSGs is associated with groups more massive than $M=10^{13} M_{\odot}$. Therefore, external processes related to cluster- or group-scale environments are not responsible for the quenching of the GAMA HDSGs. Second, in seven of eight GAMA HDSGs, the distribution of HDS spaxels covers a large portion of the galaxy, including the central regions, and there is often evidence for emission lines associated with shocks or AGN activity (Figure 8). In stark contrast, more than half $(9 / 17)$ of cluster HDSGs show evidence for star formation in their centers and the HDS spaxels are found in the outer regions of the galaxy (Figure 7). Third, the structures of the GAMA and cluster HDSGs differ significantly. The HDSGs in the clusters are more disk-like, consistent with the cluster SFGs, whereas those found in the GAMA regions tend to have Sérsic indices intermediate between the SFG and PASG populations (Figure 9). These three differences indicate that the mechanism that has quenched the star formation in the GAMA HDSGs has acted on a galaxy-wide scale, may have altered the galaxy structure, and may be internally driven. Given that our aims here are to investigate environment-related quenching, and that the GAMA HDSGs are likely quenched by internal mechanisms, a full investigation of the GAMA HDSGs is beyond the scope of this paper.
On the other hand, in the cluster regions the quenching tends to occur more locally, starting in the outer parts of the galaxy, consistent with external environment-driven quenching. Our interpretation is that the cluster HDSGs are drawn from a population of newly accreted SFGs that have very recently entered the central $0.5 R_{200}$ region close to the cluster core. During the first passage through the cluster, ram pressure stripping removes gas from the outer parts of the galaxy, leading to the outside-in quenching of star formation in the galaxies. For the remainder of this Section, we outline the evidence supporting this interpretation.

\subsection{A Recently Accreted Population}

In Section 6.2, we compared the velocity, radial, and PPS distribution of the PASGs, SFGs, and HDSGs. The analysis revealed that HDSGs reside in a tight range in projected cluster-centric distance $\left(0.15<R / R_{200}<0.6\right)$, have a significantly larger spread in velocity relative to the overall galaxy population $\left(\sigma_{\mathrm{HDS}}=1.66 \sigma_{200}\right)$, and occupy an arc-shaped region in PPS with the low-velocity HDSGs found at larger projected radii and the high-velocity HDSGs at smaller projected radii. Because many of the cluster HDSGs harbor evidence for ongoing star formation, it is intriguing that their environments differ so markedly from that of the cluster SFGs, which are more evenly distributed in radius and have a velocity dispersion similar to that of the general cluster population (although a factor of 1.2 larger than the PASGs).

These differences can be explained in a self-consistent way by considering the HDSGs as a subset of the infalling SFG population that have crossed $0.5 R_{200}$ within the last $\sim 1 \mathrm{Gyr}$, while the PASGs form a virialized population that has existed within the cluster for many gigayears. During infall, the velocity of a galaxy on a radial orbit increases with decreasing radius, peaking at pericenter, and decreasing thereafter prior to reaching zero velocity at apocenter, where it spends a significant fraction of its orbit (Gill et al. 2005). Thus, galaxies that have recently passed $0.5 r_{200,3 \mathrm{D}}$ on their first passage are more likely to be found in the higher velocity, small radius region of the PPS diagram. As a result of projection, a subset of the infalling galaxies will have the majority of their radial velocity vector aligned perpendicular to our LOS and will therefore appear in the low-velocity, small radius region of PPS.

To test the validity of this interpretation, we make use of the orbit libraries of Oman et al. (2013) and Oman \& Hudson (2016), which are used to derive probability distribution functions (PDFs) for infall times as a function of position in PPS. The orbit libraries are derived from satellites associated with cluster-scale halos in the Multidark Run $1 \mathrm{~N}$-body cosmological simulation (Prada et al. 2012). For a detailed description of the extraction of the orbit libraries, and the conversion of the full 6D phase space quantities onto 2D PPS, we refer to Oman et al. (2013) and Oman \& Hudson (2016). We rescale the simulation PPS coordinates to match our observed values as follows: for the velocity $\sqrt{3}\left|V_{\text {pec }}\right| / \sigma_{3 \mathrm{D}}=\left|V_{\text {pec }}\right| / \sigma_{1 D} \simeq\left|V_{\text {pec }}\right| / \sigma_{200}$ and for the radius $1.3 R / r_{\text {vir }} \simeq R / R_{200}$. We note that the simulated and observed $V_{\text {pec }}$ values are determined in a consistent manner, while $r_{\text {vir }}$ is determined as outlined in Bryan \& Norman (1998). We stack orbit libraries from host halos with $M_{\text {host,vir }}>10^{13} M_{\odot}$, and include satellites with halo masses $M_{\text {sat,vir }}>10^{11.9} M_{\odot}$. The limit in $M_{\text {sat,vir }}$ helps to guard against incompleteness due to artificial disruption and corresponds to a stellar mass $\log \left(M_{*} / M_{\odot}\right) \sim 10.3$, 

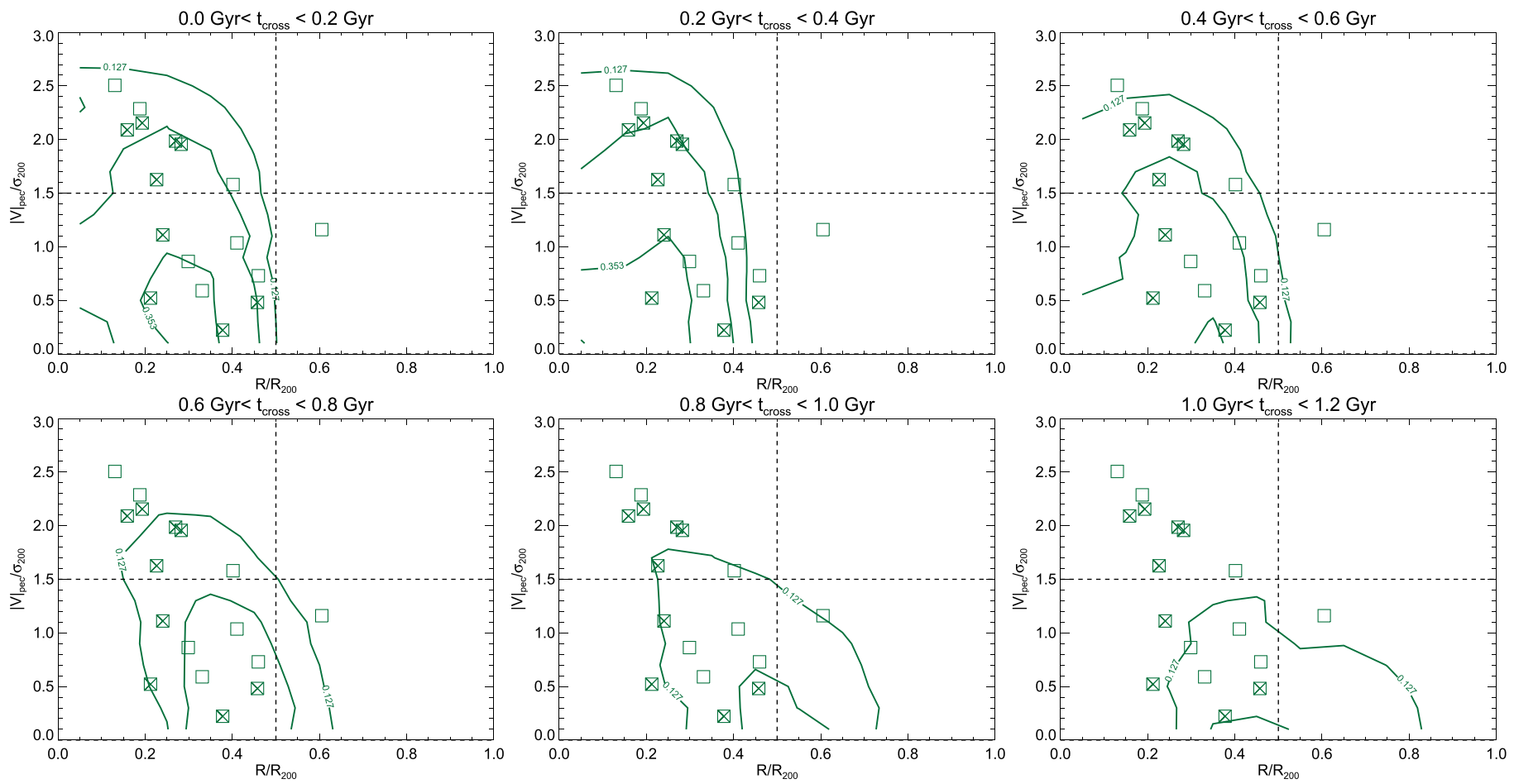

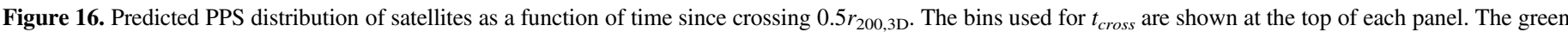

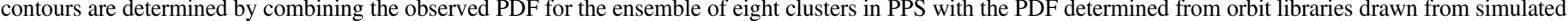

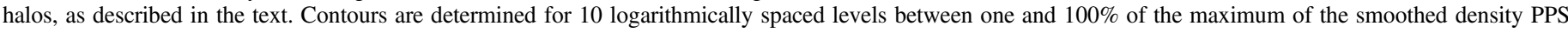

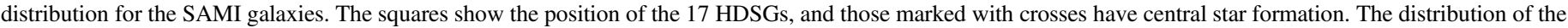
predicted PPS position for objects that have $t_{\text {cross }}<1$ Gyr is in good agreement with the distribution of HDSGs in PPS.

which is marginally higher than the limit imposed here on the SAMI galaxies (Oman et al. 2013).

Motivated by our aim of understanding whether the distribution of HDSGs in PPS is consistent with an infalling population that has recently crossed $0.5 r_{200,3 \mathrm{D}}$, infall times, $t_{\text {cross }}$, are measured from the time a satellite first crosses $0.5 r_{200,3 \mathrm{D}}$ in bins of size $0.2 \mathrm{Gyr}$, which matches the redshift zero time-stamp resolution of the simulations. We generate PDFs on a grid that spans the radial and velocity range of the SAMI galaxies used in this paper (i.e., $0 \leqslant R / R_{200} \leqslant 1$ and $\left|V_{\text {ped }}\right| / \sigma_{200} \leqslant 3$, respectively), with bins of size $\Delta R_{200}=0.1$ and $\Delta\left|V_{\text {pec }}\right| / \sigma_{200}=0.2$. Within each PPS bin, the fraction of interlopers is also determined. An interloper is defined as a halo that has $V_{\text {pec }}<2.0 \sigma_{3 \mathrm{D}}$ and $R<2.5 r_{\text {vir }}$, but has never entered the region with $r_{3 \mathrm{D}}<0.5 r_{200,3 \mathrm{D}}$ (similar to that described in Oman \& Hudson 2016). The interloper population therefore contains satellites that are first-infallers as well as halos that will never enter within $0.5 r_{200,3 \mathrm{D}}$.

In Figure 16 the green contours show the predicted number density distribution in PPS for a population of satellites with $0 \leqslant t_{\text {cross }} \leqslant 1.2 \mathrm{Gyr}$ in six time steps. The contours are generated from a grid where the simulated PDFs are multiplied by the observed density distribution of SAMI galaxies in PPS, giving the predicted number of galaxies in each PPS pixel for the time step listed on each panel. Given the relatively small sample size, to determine the observed galaxy density, we adaptively smooth the observed PPS following the method outlined in Owers et al. (2017); this smoothing helps to minimize the impact of shot-noise for the bins with few galaxies.

Comparing the contours with the distribution of HDSGs (green squares), the closest match occurs at two time steps:
$0 \leqslant t_{\text {cross }} \leqslant 0.2$ Gyr and $0.4 \leqslant t_{\text {cross }} \leqslant 0.6$ Gyr (top left and top right panel in Figure 16, respectively). The time step in the $0.2 \leqslant t_{\text {cross }} \leqslant 0.4$ Gyr range encompass pericentric passage; this period predicts a larger number of galaxies with small radius and large velocity. However, this time step predicts too many galaxies in the $R / R_{200}<0.2,\left|V_{\text {pec }}\right| / \sigma_{200}<1.5$ corner of PPS to be compatible with the observed distribution, which is completely devoid of HDSGs. At later stages when $0.6 \leqslant t_{\text {cross }} \leqslant 0.8 \mathrm{Gyr}$, the contours mainly coincide with the low-velocity HDSGs with $0.3 \leqslant R / R_{200} \leqslant 0.5$. At later times $\left(t_{\text {cross }}>0.8 \mathrm{Gyr}\right)$, the contours move toward larger radius and lower velocity and become less coherent. The approximate symmetry of the velocity evolution of infalling galaxies about pericenter causes the degeneracy in the PPS distribution for the $0 \leqslant t_{\text {cross }} \leqslant 0.2 \mathrm{Gyr}$ and $0.4 \leqslant t_{\text {cross }} \leqslant 0.6 \mathrm{Gyr}$ time steps. This degeneracy means that using PPS alone cannot distinguish whether the HDSGs in our sample belong to a population that is observed just prior to, or just following, a pericenter passage. However, we can conclude that the distribution of HDSGs is consistent with that of a population that has been accreted within $0.5 r_{200,3 \mathrm{D}}$ within the last $1 \mathrm{Gyr}$.

In Figure 17, the contours overlaid on the left, middle, and right panels are generated as described above, but now show the predicted distribution of satellites that have $t_{\text {cross }}<1 \mathrm{Gyr}$, $t_{\text {cross }}>3 \mathrm{Gyr}$, and interlopers that have yet to cross $0.5 r_{200,3 \mathrm{D}}$, respectively. Also shown are the PPS distributions of the HDSGs, PASGs, and SFGs (green squares in left panel, redfilled circles in middle panel, and blue-filled stars in right panel, respectively). The three time steps are chosen to show the PPS distribution expected of a recently accreted, virialized, and infalling population. Qualitatively, the match between the predicted and observed distributions for the three subsets is 

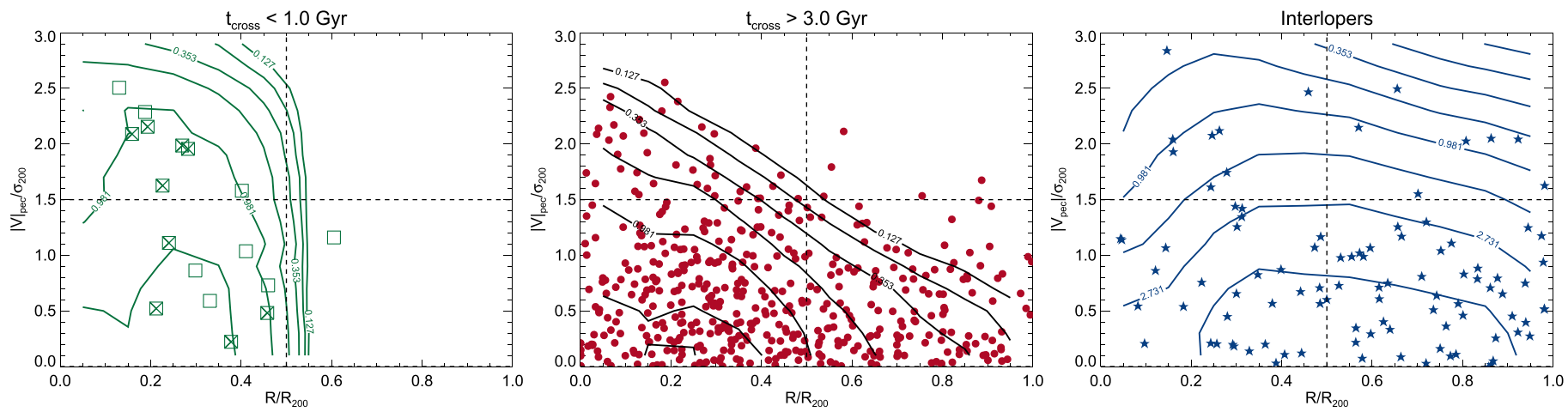

Figure 17. Comparison of the distribution of HDSGs (green squares, left panel), PASGs (red circles, middle panel), and SFGs (blue stars, right panel) with the predicted distribution of satellites with $t_{\text {cross }}<1 \mathrm{Gyr}$ (green contours, left panel), $t_{\text {cross }}>3 \mathrm{Gyr}$ (black contours, middle panel), and objects that have yet to cross $0.5 r_{200,3 \mathrm{D}}$ (blue contours, right panel). The agreement between the observed and predicted distributions provides support for the interpretation that the PASGs form a virialized population, the SFGs form an infalling population that have yet to pass pericenter, while the HDSGs are infalling objects that are observed at close to pericenter.

very good. This match supports our interpretation that the SFGs form an infalling population that have not yet passed within $0.5 r_{200,3 \mathrm{D}}$, while the HDSGs are consistent with being drawn from the population of infalling galaxies that are very close to pericenter, having recently passed $0.5 r_{200,3 \mathrm{D}}$. The PASGs form the bulk of the virialized population that has resided in the cluster for several crossing times, supporting previous results (e.g., Biviano \& Katgert 2004).

\subsection{The Outside-in Quenching of Infalling Galaxies}

In Section 6.1, we found that around half of the cluster HDSGs show evidence for central star formation, with the HDS signature found in the outer parts of those galaxies. This strongly suggests that these cluster HDSGs are being quenched in an outside-in manner. In attempting to understand the dominant process that is responsible for this outside-in quenching, there are three important pieces of evidence to consider. First, the preceding analysis suggests that the HDSGs are recent additions to the cluster environment and are very close to pericentric passage where both infall velocity and ICM density peak. Second, the analysis in Section 6.1 indicates that the structure of the galaxies is consistent with them being disklike systems, while inspection of the images in Figure 7 reveals very little visual evidence for disturbances in the stellar distribution of the galaxies. Finally, the existence of strong Balmer absorption in the outer regions suggests that the quenching must have occurred on a relatively short timescale $(<1 \mathrm{Gyr})$. Taken in concert, the evidence supports a quenching process related to hydrodynamical interactions between the galaxy's gas and the ICM that is capable of removing the gas required for star formation without disturbing the stellar component of the disk. Our interpretation is that stripping of the cold atomic $\mathrm{H} \mathrm{I}$ and molecular gas disk due to ram pressure stripping (Gunn \& Gott 1972) is the most likely mechanism responsible for the quenching. However, it is worth considering why other processes commonly attributed to cluster-related quenching of star formation are less likely.

We can immediately rule out strangulation as being responsible for the production of the HDSGs. Strangulation quenches star formation via the removal of the hot gas halo reservoir that replenishes the cold gas required for star formation (Larson et al. 1980). This process is slow acting, requiring several gigayears to quench a galaxy, and simulations show that the quenching is a gradual disk-wide process resulting in spiral-like PASGs (Bekki et al. 2002). These predictions are inconsistent with the existence of the young, localized HDS regions observed here that indicate a more rapid removal of gas. While the hot gas reservoirs may have been removed from the HDSGs during the early phases of infall, it is not the dominant quenching mechanism currently at play.

Similarly, we can rule out quenching due to major galaxygalaxy interactions and mergers. First, the large relative velocities between the galaxies in the cores of clusters mean that major mergers are rare in cluster cores and are more likely to occur in the cluster outskirts $\left(R>R_{200}\right.$; Ghigna et al. 1998; Moran et al. 2007). Second, inspection of the galaxy images shown in Figure 7 reveals that only one galaxy (9016800216) shows obvious tidal-like features in the stellar distribution, whereas the majority of the cluster HDSGs appear to have relatively unperturbed stellar morphologies with no evidence for mergers. Multiple, high-speed tidal interactions between galaxies (harassment; Moore et al. 1996) and tidal forces due to the cluster potential (Byrd \& Valtonen 1990) can enhance central star formation and disrupt galaxy disks. However, we do not see any evidence for abnormal SFRs (as determined by their $\mathrm{EW}(\mathrm{H} \alpha)$ in Section 6.2.1). Furthermore, it is unlikely that tidal interactions will preferentially remove gas without also affecting the stellar disk, while Boselli \& Gavazzi (2006) find that tidal interactions with the cluster potential are unlikely to remove large amounts of $\mathrm{HI}$ gas. While these processes are unlikely to be responsible for the appearance of the HDSGs features, their cumulative effects may well have played a role in aiding the process of gas stripping that led to the current state.

On the other hand, stripping of the cold gas via ICM interactions naturally explains the observations because they can remove gas in an outside-in manner without significantly affecting the stellar structure of the galaxy (Boselli \& Gavazzi 2006). Simulations predict that stripping can be a multistage process with a continuous phase that slowly removes gas via viscous stripping, and a more rapid phase that generally occurs near a pericentric passage where ram pressure stripping can displace the $\mathrm{HI}$ gas from the stellar disk on timescales of less than a few hundred megayears (Nulsen 1982; Quilis et al. 2000; Schulz \& Struck 2001; Roediger \& Brüggen 2006, 2007; Roediger 2009; Bekki 2014; Jung et al. 2018). Recent observational evidence has indicated that, as well as stripping the atomic $\mathrm{HI}$ gas component, strong ram pressure stripping may also remove the molecular components of the galaxy 
within the optical disk, leading to quenching of star formation (Cortese et al. 2012; Boselli et al. 2014a; Lee et al. 2017; Noble et al. 2019). This rapid local stripping would lead to localized HDS signatures in the outer parts of the disk, as observed in our HDSG sample.

We can estimate the expected effects of ram pressure stripping using the analytical prescription of Gunn \& Gott (1972), and following a procedure similar to that performed by (Cortese et al. 2007, see also Boselli \& Gavazzi 2006; Hernández-Fernández et al. 2014; Jaffé et al. 2015, 2018), which estimates the stripping radius, $R_{\text {strip }}$, by determining the radius at which ram pressure overcomes the gravitational restoring force per unit area of the galaxy. The ram pressure is estimated as

$$
P_{\text {ram }}=\rho_{\text {ICM }} v_{\text {gal }}^{2}
$$

where $\rho_{\mathrm{ICM}}$ is the ICM density, and $v_{\text {gal }}$ is the relative velocity difference between the ICM and the galaxy. To determine $\rho_{\mathrm{ICM}}$, we make use of the results obtained by Ghirardini et al. (2019), who fitted the scaled electron density, $n_{e}\left(r / r_{500}\right) / E(z)^{2}$, profiles of 12 clusters using the analytical form defined in Equation (3) of Vikhlinin et al. (2006). We use the best-fitting parameter estimates given in Table 3 of Ghirardini et al. (2019) to determine the ICM density at radius $r$ as $\rho_{\mathrm{ICM}}(r)=\mu_{\mathrm{H}} m_{p} n_{e}(r) / \nu_{\mathrm{H}}$, where $m_{p}$ is the proton mass, $\nu_{\mathrm{H}}=1.17366$ is the number of electrons per hydrogen atom, and $\mu_{\mathrm{H}}=1.34732$ is the mean particle weight per hydrogen atom, all assuming an ICM with metallicity $Z=0.3 Z_{\odot}$ (Grevesse \& Sauval 1998). Because we normalize our projected radii using $R_{200}$, we rescale as $R_{500} \simeq 0.65 R_{200}$ (Reiprich et al. 2013).

It is not possible to determine $v_{\text {gal }}$ from observations; the LOS velocity that we measure, $v_{\text {pec }}$, provides a lower limit on this value. Likewise, the projected radius, $R$, also provides a lower limit on the 3D position $r_{3 \mathrm{D}}$, meaning that both the estimate for $\rho_{\mathrm{ICM}}$ and $v_{\text {gal }}$ have some uncertainty associated with them owing to projection effects. However, as outlined in Section 7.1, the distribution of the HDSGs in PPS is consistent with an infalling population that recently crossed $0.5 R_{200}$ and is likely to be on close to radial orbits that follow the infall velocity profile of the cluster. We can, therefore, approximate $v_{\text {gal }}$ using the infall velocity $v_{\text {inf }}(r)=\sqrt{2 G M(<r) / r}$, where $M$ $(<r)$ is the mass within radius $r$, which is determined from the caustics mass profile derived in Owers et al. (2017). We produce three estimates of $P_{\text {ram }}$ for each of the HDSGs: the first estimates $P_{\text {ram }}\left(R, v_{\text {pec }}\right)=\rho_{\text {ICM }}(R) v_{\text {pec }}^{2}$ at the observed position in PPS, the second $P_{\text {ram }}\left(R, v_{\text {inf }}(R)\right)=\rho_{\text {ICM }}(R) v_{\text {inf }}(R)^{2}$ assumes the projected $R=r$ and estimates the infall velocity, $v_{\text {inf }}(R)$ at that position, and the third $P_{\text {ram }}\left(0.5 R_{200}\right)=\rho_{\text {ICM }}(0.5$ $\left.R_{200}\right) v_{\text {inf }}\left(0.5 R_{200}\right)^{2}$ estimates ram pressure experienced upon entering $0.5 R_{200}$ at the infall velocity. These estimates span a range of $P_{\text {ram }}$ values that a galaxy is expected to experience having entered within $0.5 R_{200}$; the minimum occurs at $P_{\text {ram }}(0.5$ $R_{200}$ ), and an upper limit at the position in PPS of $P_{\text {ram }}\left(R, v_{\text {inf }}(R)\right)$, assuming that the galaxy has not yet past pericenter.

The restoring force per unit area for each HDS galaxy is:

$$
\Pi=2 \pi G \Sigma_{\text {stars }} \Sigma_{\text {gas }} .
$$

where $\Sigma_{\text {stars }}$ and $\Sigma_{\text {gas }}$ are the surface density profiles for the stars and gas, respectively, which are assumed to follow an exponentially declining profile estimated following Domainko et al. (2006):

$$
\Sigma(r)=\Sigma_{0} e^{-r_{g} / R_{d}}
$$

where $R_{d}$ is the disk scale length, $\Sigma_{0}$ is the central surface density, and $r_{g}$ is the distance from the center of the galaxy. For the stars, an exponentially declining profile is justified given that the majority of the HDSGs have $r$-band Sérsic indices $n_{\text {ser }} \simeq 1$ and are, therefore, disk-dominated systems (Figure 9). The stellar central density is $\Sigma_{0}^{*}=M_{*} /\left(2 \pi R_{d}^{2}\right)$, where we have assumed that the stellar mass proxy, $M_{*}$ is dominated by the disk component of the galaxy. The stellar disk scale length $R_{d}^{*}=0.59 r_{e}$, where $r_{e}$ is the effective radius measured in Section 2.2.1. Estimates for the gas scale length, $R_{d, \text { gas }}$, and central density, $\Sigma_{0, \text { gas }}=M_{\mathrm{gas}} /\left(2 \pi R_{d, \text { gas }}^{2}\right)$, are less wellconstrained for the HDSGs. For the scale length, we follow Boselli \& Gavazzi (2006) and assume that $R_{d, g a s} \simeq 1.8 R_{d}^{*}$. For the gas mass, we use the $M_{\mathrm{gas}} / M^{*}$ scaling relations provided by Catinella et al. (2018) to estimate the total $M_{\text {gas }}$. We assume that our HDSGs have originated from SFGs on the blue cloud, which has $N U V-r \sim 3$ in Figure 6. According to Table 2 in Catinella et al. (2018), galaxies with this color have $M_{\text {gas }} \simeq 0.4 M^{*}$, and we therefore use this for our estimates of $M_{\text {gas }}$ for each HDS galaxy.

We can now combine Equations (2) and (3) to measure the stripping radius

$$
R_{\text {strip }}=\frac{r_{e}}{2.64} \times \ln \left(\frac{0.4 G M_{*}^{2}}{2.47 P_{\mathrm{ram}} r_{e}^{4}}\right)
$$

for each of our three different $P_{\text {ram }}$ values and for each HDSG. We note that the Gunn \& Gott (1972) analytical approximation for $R_{\text {strip }}$ assumes that the galaxy is traversing the ICM face-on. However, Roediger \& Brüggen (2007) showed that there is good agreement between analytical and simulated $R_{\text {strip }}$ estimates, except for galaxies moving close to edge-on though the ICM. The results are shown as ellipses overlaid on the $\mathrm{EW}\left(\mathrm{H}_{\alpha}\right)$ maps in Figure 7, where $R_{\text {strip }}$ is represented as the major axis of the ellipse with PA and ellipticity determined from the Sérsic fits in Section 2.2.1. The gray dashed, blue dotted-dashed, and purple solid ellipses show the results for $P_{\text {ram }}\left(0.5 R_{200}\right), P_{\text {ram }}\left(v_{\text {pec }}, R\right)$, and $P_{\text {ram }}\left(v_{\text {inf }}(R), R\right)$, respectively. Also overlaid on the $\operatorname{EW}\left(\mathrm{H}_{\alpha}\right)$ maps in Figure 7 are black contour levels drawn at $\mathrm{EW}\left(\mathrm{H}_{\alpha}\right)=3 \AA$ including only spaxels that are classified as SF, INT, or wSF, and therefore show the region at which the star formation is truncated. In Figure 18 we show the semimajor axis of the $\operatorname{EW}\left(\mathrm{H}_{\alpha}\right)=3 \AA$ region, $R_{\mathrm{H} \alpha}$, which is determined by fitting an ellipse to the black contours shown in Figure 7, versus the three measures of $R_{\text {strip. }}$ The $R_{\text {strip }}$ estimates for the $P_{\text {ram }}\left(0.5 R_{200}\right)$ and $P_{\text {ram }}\left(R, v_{\text {pec }}\right)$ (blue dotteddashed and red dashed lines in Figure 18) values are generally much larger than $R_{\mathrm{H} \alpha}$. On the other hand, for eight of nine HDSGs with central star formation, the $R_{\text {strip }}$ values estimated using $P_{\text {ram }}\left(v_{\text {inf }}(R), R\right)$ (shown as black crosses in Figure 18) are within a factor $\lesssim 1.5$ of the $R_{\mathrm{H} \alpha}$. This agreement supports the hypothesis that the ram pressure stripping encountered by these 


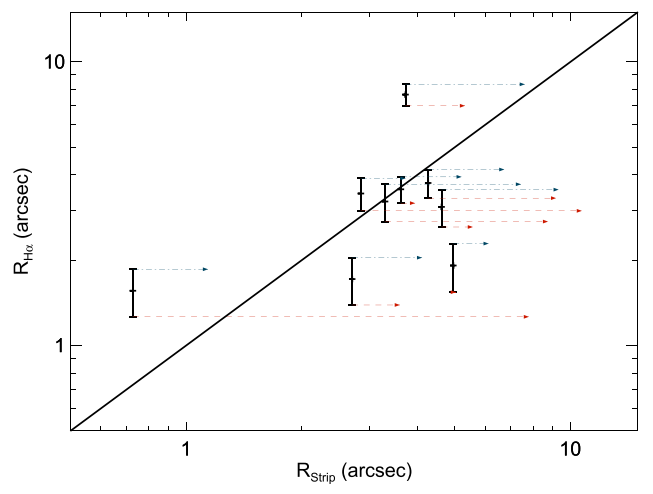

Figure 18. Extent of the $\mathrm{H} \alpha$ emission driven by star formation, $R_{\mathrm{H} \alpha}$, vs. predictions for the stripping radius $R_{\text {strip }}$ determined from Equation (5). The black crosses show the $R_{\mathrm{H} \alpha}$ vs. $R_{\text {strip }}$ for the $P_{\text {ram }}\left(v_{\text {inf }}(R), R\right)$ ram pressure estimate at the projected position of the galaxy at the infall velocity. The blue dotted-dashed and red dashed arrows show $R_{\text {strip }}$ values estimated for the ram pressure estimated at $P_{\text {ram }}\left(0.5 R_{200}\right)$ and $P_{\text {ram }}\left(R, v_{\text {pec }}\right)$, which are the ram pressure estimates using the infall velocity at $0.5 R_{200}$, and the observed position in PPS, respectively. The black solid line shows the one-to-one relation. In eight of nine cases, the $R_{\text {strip }}$ estimated for $P_{\text {ram }}\left(v_{\text {inf }}(R), R\right)$ is within a factor $\lesssim 1.5$ of $R_{\mathrm{H} \alpha}$.

HDSGs on first infall is capable of removing the gas disk leading to the observed truncation of star formation.

The above analysis provides support for ram pressure stripping in the HDSGs with central star formation. However, inspection of the $R_{\text {strip }}$ ellipses for the eight HDSGs with no evidence for ongoing star formation indicates that the predicted ram pressure at their current positions is insufficient to completely strip their gas. In this regard, it is important to note that the stripping radius estimates given by Equation (5) do not account for past stripping (e.g., if those galaxies have previously passed pericenter where they experienced peak ram pressure). Figure 16 shows that six of eight of the NSF HDSGs are located at larger projected distances and smaller LOS velocities when compared with the remaining HDSGs. We speculate that the majority of these galaxies have passed pericenter and are currently outbound toward apocenter. This interpretation is supported by the simulations presented in Figure 16, which shows that the expected position of the postpericenter, outbound satellites in the 0.6-1.0 Gyr panels is consistent with the positions of the NSF HDSGs. In this scenario, the strong ram pressure experienced during pericenter would have completely stripped the gas from the galaxies, leading to their completely quenched state.

\subsection{Comparison with Previous Work}

The results and analysis presented in this paper lead us to conclude that ram pressure stripping plays an important role in quenching infalling SF disk galaxies on their first passage through the cluster core. This conclusion is supported by observations in the nearby Virgo cluster, where Koopmann \& Kenney (2004b) found that around half of the spiral galaxies in the Virgo cluster exhibited truncated $\mathrm{H} \alpha$ disks, with little evidence for any disturbance in the stellar disks, and that the majority of the truncated spirals were found close to the core of the cluster. Moreover, Chung et al. (2009b) and Chung et al. (2007) found that the H I distributions of galaxies in the core of Virgo $\left(R / R_{200} \lesssim 0.3\right.$, where $R_{200}=1550 \mathrm{kpc}$; Ferrarese et al. 2012) are often asymmetric and always truncated with respect to the stellar disk, while many galaxies in the $0.4 \leqslant$
$R / R_{200} \leqslant 0.7$ range exhibit one-sided H I tails that point away from the center of Virgo. These observations provide evidence that ram pressure begins to strip the $\mathrm{H}$ I disk of galaxies as they fall into Virgo, and removes much of the HI disk during pericenter, leading to truncated star formation.

Our results are broadly consistent with those of Crowl \& Kenney (2008), where the stellar population ages in the outer disks of around half of their sample of truncated Virgo spirals were consistent with the timescales expected due to ram pressure stripping experienced near pericenter. However, Crowl \& Kenney (2008) also find evidence that a subset of their galaxies must have been stripped outside of the cluster core; their interpretation is that tidal interactions and enhanced ram pressure stripping due to bulk motions and ICM substructure may be responsible for stripping at larger cluster-centric distances. In contrast with the findings of Crowl \& Kenney (2008), we find only one HDSG outside its cluster core $\left(R>0.5 R_{200}\right)$. However, it is worth noting that only a handful of the Crowl \& Kenney (2008) galaxies would pass our $\mathrm{EW}(\mathrm{H} \delta)<-3 \AA$ selection criteria, so care needs to be taken in directly comparing our results with theirs. It is possible that our selection criteria bias the selection to include only those objects undergoing the most rapid stripping, and that there are galaxies with truncated star formation that are not included in our sample. Indeed, Figure 10 reveals that there are 13 SFGs that have $C_{\mathrm{H} \alpha \text {,cont }}$ in the same range as the HDSGs with central star formation; half of these SFGs have $R>0.5 R_{200}$. We will investigate the properties of these SFGs with centrally concentrated star formation in a forthcoming paper.

Cortese \& Hughes (2009) investigated a sample of local transition galaxies that are located in the green valley in the NUV-H diagram. They found that the majority of the transition galaxies are H I-deficient and located in the Virgo cluster. The Virgo transition galaxies are disk-like, have radial and velocity distribution that are consistent with that of an infalling population, and around half show evidence for star formation at their centers. These pieces of evidence led Cortese \& Hughes (2009) to conclude that ram pressure stripping was responsible for the quenching of star formation in the Virgo transition galaxies, consistent with the results presented here for our HDSGs. The importance of ram pressure stripping in quenching star formation in Virgo has found much support from many other avenues (Cortese et al. 2011, 2012; Boselli et al. 2014a, 2014b, 2016). The results presented here provide support for these previous results, and extend them to environments beyond the nearby Virgo cluster.

The radial distribution of the HDSGs within our cluster ensemble is consistent with that found in Paccagnella et al. (2017), who investigated the environments of a large sample of HDSGs $^{29}$ selected from the WINGS (Fasano et al. 2006). In agreement with the results in Figure 12, Paccagnella et al. (2017) found that the ratio of HDSGs to the total number of active galaxies (emission line and HDSGs) increases markedly toward the cluster center. In contrast with our results, Paccagnella et al. (2017) did not find significant differences when comparing the kinematics and PPS distribution of the passive and HDS sample. However, their sample of HDSGs with $\mathrm{EW}(\mathrm{H} \delta)>8 \AA$ did show both a significantly higher

\footnotetext{
${ }^{29}$ Paccagnella et al. (2017) use the nomenclature "poststarburst" and "strong poststarburst" to describe their samples. We note that their poststarburst sample is selected to have $3<\mathrm{EW}(\mathrm{H} \delta)<8 \AA$, while galaxies with $\mathrm{EW}(\mathrm{H} \delta)>8 \AA$ are strong poststarbursts.
} 
velocity dispersion than the PASGs, as well as a tendency to be clustered at $\left|v_{\text {pec }}\right| / \sigma_{200} \simeq 1.5$ and $R / R_{200} \simeq 0.25$, in agreement with the results shown in Figure 13 and 14.

In comparing the results of Paccagnella et al. (2017) with those presented here, a crucial caveat is that their selection relies on single-fiber spectroscopy. At the redshifts of the WINGS clusters $(0.04<z<0.07$ Moretti et al. 2017), the spectra cover an area that subtends a physical scale of only $1.3-2.8 \mathrm{kpc}$ in diameter. This means that in order to be included in their HDS sample, the strong Balmer absorption signature must be present within the very central parts of the galaxy. Furthermore, Paccagnella et al. (2017) exclude from their HDS sample all galaxies that have [OII], [O III] $(\lambda 5007), \mathrm{H} \beta$, or $\mathrm{H} \alpha$ detected in emission. Inspection of Figure 7 reveals that only two of our cluster HDSGs have central spectra that meet these criteria. Further investigation of the spectral properties within the central $2^{\prime \prime}$ for the remaining SAMI cluster galaxies reveals that no other objects would meet the HDS criteria of Paccagnella et al. (2017). Thus, fewer than $0.5 \%$ of our sample would be classified as HDS in single-fiber surveys, consistent with previous results for local clusters where HDSGs with $\log \left(M_{*} / M_{\odot}\right)>10$ are rare (Poggianti et al. 2004; Gavazzi et al. 2010; Boselli et al. 2014b). This fraction is, however, substantially lower than that reported by Paccagnella et al. (2017), who found that $7.3 \%$ of $\log \left(M_{*} / M_{\odot}\right)>9.8$ cluster galaxies are classified as HDSGs. We also note that a large fraction of the HDSGs in Paccagnella et al. (2017) lie on the red-sequence (see their Figure 3), whereas the majority of our sample lie blueward of the red-sequence (Figure 6). Thus, while there are some consistencies between the trends with environment seen in our HDSGs and those reported by Paccagnella et al. (2017), it is clear that the two samples are unlikely to be tracing the same population of transition galaxies.

The coherent arc-like distribution of HDSGs in PPS shown in Figure 14 is strikingly similar to that found in a sample of $z \sim 1$ clusters by Muzzin et al. (2014). Comparing the distribution of their HDSGs to four simulated clusters, Muzzin et al. (2014) find that the galaxies were likely quenched within $500 \mathrm{Myr}$ after crossing $0.5 R_{200}$, consistent with our interpretation that the SAMI HDSGs are recent arrivals to the region within $0.5 R_{200}$. Muzzin et al. (2014) favor the fast-acting ram pressure stripping of the cold gas as the likely quenching mechanism, although they could not conclusively rule out slower processes such as starvation/strangulation due to the removal of the hot gas reservoir. If the HDSGs presented here are local analogs of those in Muzzin et al. (2014), then they may offer a valuable and more accessible insight into the quenching processes operating in higher redshift clusters. In this vein, we note that the HDSGs in Muzzin et al. (2014) are selected to have weak [OII] emission, indicating that they are fully quenched, whereas many of our HDSGs are only partially quenched. This partial quenching indicates that our HDSGs are at an earlier quenching phase than those detected in Muzzin et al. (2014). If so, then the relative consistency of the coherent phase space positions between the two samples supports a quenching scenario driven by ram pressure stripping during the core passage phase in both high- and low-redshift clusters.

The rapid phase of the "delayed-then-rapid" quenching scenario (Wetzel et al. 2012) has been attributed to very efficient, complete quenching that occurs within $\sim 1 \mathrm{Gyr}$ of pericentric passage, likely due to the effects of ram pressure stripping (Oman \& Hudson 2016). The HDSGs observed here provide support for a substantial fraction of infalling SFGs being affected by ram pressure stripping at close to pericenter. In particular, Table 3 shows that the fraction of HDSGs is highest in the $R / R_{200}<0.5, \mid V_{\text {pec }} / \sigma_{200}>1.5$ portion of the PPS diagram, where they make up $15 \%$ of the total population, and $50 \%$ of the population that show evidence for recent or ongoing star formation. The simulations in Section 7.1 indicate that this PPS region contains the highest fraction of recently accreted satellites, with $25 \%$ having $t_{\text {cross }} \leqslant 1 \mathrm{Gyr}, 17 \%$ with $t_{\text {cross }}>1 \mathrm{Gyr}$, while the remaining $58 \%$ of satellites have not yet crossed $0.5 r_{200,3 \mathrm{D}}$. Therefore, the SFGs seen in this region of PPS that do not show evidence for quenching can be accounted for by projection effects, indicating that the majority of SFGs that pass within $0.5 r_{200,3 \mathrm{D}}$ will begin the quenching process.

While many of the HDSGs are completely quenched, thereby supporting a rapid quenching phase within $1 \mathrm{Gyr}$ of pericenter, a substantial fraction of the HDSGs are not yet completely quenched. These partially quenched galaxies may be either caught just prior to peak ram pressure experienced at pericenter, or they are outgoing and were not fully quenched at pericenter. In principle, these two scenarios can be distinguished by deep, highresolution narrow-band $\mathrm{H} \alpha$ or UV imaging that can reveal onesided, extraplanar tails of ionized gas and young stars, such as those seen in galaxies in nearby clusters (Smith et al. 2010; Yagi et al. 2010; Boselli et al. 2018; Gavazzi et al. 2018). The kinematics and radial distributions of these tailed galaxies indicate that they are an infalling population at close to pericenter, consistent with our HDSGs. The additional information provided by the orientation of the tails, which are found to generally point away from the cluster center, strongly indicates that the galaxies are observed prior to pericenter, and that stripping is occurring on the first passage. We note that there is some tentative evidence for one-sided or extraplanar $\mathrm{H} \alpha$ emission in several of our HDSGs (e.g., 9016800074, 9008500100, 9008500107, and 9011900084 in Figure 7). However, it is difficult to draw strong conclusions based on those few galaxies because the emission is often close to the edge of the SAMI FOV, meaning that it is not clear whether the $\mathrm{H} \alpha$ morphology is tail-like. Moreover, for a large fraction of our sample the SAMI FOV is too small to probe extraplanar emission and, therefore, to detect tails.

\section{Summary and Conclusions}

The spatially resolved spectroscopy provided by the SAMIGS has allowed for a new method of selecting galaxies that show evidence for ongoing or very recent cessation of star formation, or quenching. Our method outlined in Section 5 uses the fraction of spaxels that show strong Balmer absorption in the absence of emission lines associated with ongoing star formation. This selection allows for the detection of localized quenching that, for many of the galaxies in our sample, would not be observed in single-fiber spectroscopic surveys. Using this new technique, we selected 25 HDSGs from the SAMI-GS, with 17 found in the cluster regions and eight found in the GAMA regions. We have focused on investigating the environments of the cluster HDSGs with the primary aim of disentangling which environment-related mechanisms were most important in shutting down star formation. Our key results can be summarized as follows: 
1. Galaxies with HDS signatures are rare overall, making up only $\sim 2 \%$ of the $\log \left(M_{*} / M_{\odot}\right)>10$ galaxy population. However, they are significantly more common among the population of galaxies that show evidence for recent or ongoing star formation in the cluster regions (15\%) when compared with the lower density GAMA regions $(2 \%)$. Notably, only two of 17 of the cluster HDSGs would be identified in single-fiber surveys; the majority of the HDS regions are found in the outer parts of the galaxy, away from the center.

2. The HDSGs found in the GAMA regions are different from those found in the cluster regions in several important ways. First, they are not associated with massive $\left(M_{200}>10^{13} M_{\odot}\right)$ groups in the GAMA regions. Second, the majority (7/8) of the GAMA HDSGs show little evidence for ongoing star formation, whereas many of the cluster HDSGs show central star formation $(9 / 17)$. Third, the structure of the cluster HDSGs is generally disk-like $\left(n_{\text {ser }}<2\right)$, whereas the majority of the GAMA HDSGs have $n_{\text {ser }}>2$.

3. Focusing on the cluster regions, we find that there are significant differences between the radial, velocity, and PPS distributions of the HDSGs when compared to the PASGs and SFGs. The cluster HDSGs are exclusively found to have cluster-centric distances $R / R_{200} \leqslant 0.6$, and have a larger velocity dispersion than the general cluster population $\left(\sigma_{\mathrm{HDS}}=1.66_{-0.25}^{+0.29} \sigma_{200}\right)$. The distribution of HDSGs in the PPS reveals a coherent arc-like structure, where the HDSGs with smaller cluster-centric distances have higher velocities, and those at larger cluster-centric distances have smaller velocities.

4. By comparing with the simulated orbit libraries derived from clusters selected from cosmological $N$-body simulations by Oman et al. (2013), we find that the distribution of HDSGs in PPS is consistent with that expected of a population of infalling galaxies that have entered within $0.5 r_{200,3 \mathrm{D}}$ within the last $1 \mathrm{Gyr}$. We find that the SFG PPS distribution is consistent with an infalling population that is yet to pass $0.5 r_{200,3 \mathrm{D}}$, while the PASG PPS distribution is consistent with a virialized population.

5. For the eight of nine of the cluster HDSGs with central star formation, the extent of the $\mathrm{EW}(\mathrm{H} \alpha)$ emission can be explained by outside-in quenching due to ram pressure stripping.

We conclude that the HDSGs in the cluster regions consist of a population of infalling SFGs that are close to pericenter and are currently being quenched due to ram pressure stripping. On the other hand, the quenching of the star formation in the GAMA HDSGs is unlikely to be associated with large-scale environment processes and may be internally driven. We note that, by definition, our selection biases us toward selecting objects that have had their star formation quenched within the last $\sim 1.5 \mathrm{Gyr}$ and, therefore, toward selecting for processes that quench star formation on relatively short timescales.

While our results show that ram pressure stripping is likely a very important mechanism for quenching in clusters, more subtle effects on star formation due to, for example, starvation may also gradually lower star formation during the early phases of infall. There is also the key question of the future evolution of the HDSGs. Do they maintain some star formation at their centers as they head to apocenter, or are they completely stripped during pericenter? In future work, we will address these important questions by using the cluster portion of the SAMI-GS to perform a more comprehensive investigation of the current and recent star formation of cluster galaxies and the relation to the cluster environment. We note that the nextgeneration HECTOR Galaxy Survey (Bryant et al. 2016) will extend the SAMI-GS by providing resolved spectroscopy for a much larger sample of cluster galaxies, which will include more high-mass halos $\left(M_{200}>10^{14.5} M_{\odot}\right)$ and also galaxies in the cluster outskirts (up to $2 R_{200}$ ). This extended survey will enable the study of important regions where preprocessing may be important, as well as probing the regions where "backsplash" galaxies are most commonly found (Balogh et al. 2000; Gill et al. 2005).

We thank the anonymous referee for their comments that have helped to improve this paper. M.S.O. acknowledges the funding support from the Australian Research Council through a Future Fellowship (No. FT140100255). M.H. acknowledges support from an NSERC Discovery Grant, from the Australian Astronomical Observatory Distinguished Visitor Scheme and from the Australian Research Council Centre of Excellence for All Sky Astrophysics in 3 Dimensions (ASTRO 3D) also as a Distinguished Visitor. K.O. received support from VICI grant No. 016.130.338 of the Netherlands Foundation for Scientific Research (NWO). J.B.H. is supported by an ARC Laureate Fellowship that funds Jesse van de Sande and an ARC Federation Fellowship that funded the SAMI prototype. S.B. acknowledges the funding support from the Australian Research Council through a Future Fellowship (No. FT140101166). J.J.B. acknowledges support of an Australian Research Council Future Fellowship (No. FT180100231). L.C. is the recipient of an Australian Research Council Future Fellowship (No. FT180100066) funded by the Australian Government. J.vd.S. is funded under BlandHawthorn's ARC Laureate Fellowship (No. FL140100278). C.F. acknowledges funding provided by the Australian Research Council (Discovery Projects No. DP150104329 and No. DP170100603, and Future Fellowship No. FT180100495), and the Australia-Germany Joint Research Cooperation Scheme (UADAAD). B.G. is the recipient of an Australian Research Council Future Fellowship (No. FT140101202). Support for A.M.M. is provided by NASA through Hubble Fellowship grant No. HSTHF2-51377 awarded by the Space Telescope Science Institute, which is operated by the Association of Universities for Research in Astronomy, Inc., for NASA, under contract No. NAS5-26555. N.S. acknowledges support of a University of Sydney Postdoctoral Research Fellowship. S.K.Y. acknowledges support from the Korean National Research Foundation (grant No. 2017R1A2A1A05001116) and by the Yonsei University Future Leading Research Initiative (grant No. 2015-22-0064). This study was performed under the umbrella of the joint collaboration between Yonsei University Observatory and the Korean Astronomy and Space Science Institute.

The SAMI Galaxy Survey is based on observations made at the Anglo-Australian Telescope. The Sydney-AAO Multiobject Integral-field spectrograph (SAMI) was developed jointly by the University of Sydney and the Australian Astronomical Observatory. The SAMI input catalog is based on data taken from the Sloan Digital Sky Survey, the GAMA Survey, and the VST/ATLAS Survey. The SAMI Galaxy Survey is supported by the Australian Research Council Centre of Excellence for All Sky Astrophysics in 3 Dimensions (ASTRO 3D), through project No. CE170100013, the Australian 
Research Council Centre of Excellence for All Sky Astrophysics (CAASTRO), through project No. CE110001020, and other participating institutions. The SAMI Galaxy Survey website is http://sami-survey.org/.

GAMA is a joint European-Australasian project based on a spectroscopic campaign using the Anglo-Australian Telescope. The GAMA input catalog is based on data taken from the Sloan Digital Sky Survey and the UKIRT Infrared Deep Sky Survey. Complementary imaging of the GAMA regions is being obtained by a number of independent survey programs, including GALEX MIS, VST KiDS, VISTA VIKING, WISE, Herschel-ATLAS, GMRT, and ASKAP providing UV to radio coverage. GAMA is funded by the STFC (UK), the ARC (Australia), the AAO, and the participating institutions. The GAMA website is http://www.gama-survey.org/. Based on observations made with ESO Telescopes at the La Silla Paranal Observatory under program ID No. 177.A-3016. Based on data products (VST/ATLAS) from observations made with ESO Telescopes at the La Silla Paranal Observatory under program ID No. 177.A-3011(AJ). This paper includes data that have been provided by AAO Data Central (datacentral.org.au).

Facility: AAT (SAMI).

\section{ORCID iDs}

Matt S. Owers (ํ) https://orcid.org/0000-0002-2879-1663 Michael J. Hudson (1) https://orcid.org/0000-0002-1437-3786 Joss Bland-Hawthorn 주 https://orcid.org/0000-00017516-4016

S. Brough 지 https://orcid.org/0000-0002-9796-1363 Luca Cortese (10) https://orcid.org/0000-0002-7422-9823 Warrick J. Couch (1) https://orcid.org/0000-0001-5005-3125 Jesse van de Sande (10 https://orcid.org/0000-0003-2552-0021 Christoph Federrath (1) https://orcid.org/0000-0002-0706-2306 Brent Groves (i) https://orcid.org/0000-0002-9768-0246 A. M. Hopkins (1) https://orcid.org/0000-0002-6097-2747 Anne M. Medling (iㅏ https://orcid.org/0000-0001-7421-2944 Samuel N. Richards (1) https://orcid.org/0000-0002-5368-0068 Nicholas Scott (1) https://orcid.org/0000-0001-8495-8547 Dan S. Taranu (1) https://orcid.org/0000-0001-6268-1882 Sukyoung K. Yi i https://orcid.org/0000-0002-4556-2619

\section{References}

AAO software team, 2015, 2dfdr: Data Reduction Software, Astrophysics Source Code Library, ascl:1505.015

Ahn, C. P., Alexandroff, R., Allende Prieto, C., et al. 2012, ApJS, 203, 21

Alatalo, K., Cales, S. L., Rich, J. A., et al. 2016, ApJS, 224, 38

Allen, J. T., Croom, S. M., Konstantopoulos, I. S., et al. 2015, MNRAS, 446, 1567

Allen, J. T., Green, A. W., Fogarty, L. M. R., et al. 2014, SAMI: Sydney-AAO Multi-object Integral Field Spectrograph Pipeline, Astrophysics Source Code Library, ascl:1407.006

Baldry, I. K., Liske, J., Brown, M. J. I., et al. 2018, MNRAS, 474, 3875

Baldwin, J. A., Phillips, M. M., \& Terlevich, R. 1981, PASP, 93, 5

Balogh, M., Eke, V., Miller, C., et al. 2004, MNRAS, 348, 1355

Balogh, M. L., Morris, S. L., Yee, H. K. C., Carlberg, R. G., \& Ellingson, E. 1999, ApJ, 527, 54

Balogh, M. L., Navarro, J. F., \& Morris, S. L. 2000, ApJ, 540, 113

Barsanti, S., Girardi, M., Biviano, A., et al. 2016, A\&A, 595, A73

Barsanti, S., Owers, M. S., Brough, S., et al. 2018, ApJ, 857, 71

Beers, T. C., Flynn, K., \& Gebhardt, K. 1990, AJ, 100, 32

Bekki, K. 1999, ApJL, 510, L15

Bekki, K. 2009, MNRAS, 399, 2221

Bekki, K. 2014, MNRAS, 438, 444

Bekki, K., Couch, W. J., \& Shioya, Y. 2002, ApJ, 577, 651

Belfiore, F., Maiolino, R., Maraston, C., et al. 2016, MNRAS, 461, 3111
Belfiore, F., Maiolino, R., Maraston, C., et al. 2017, MNRAS, 466, 2570 Bellhouse, C., Jaffé, Y. L., Hau, G. K. T., et al. 2017, ApJ, 844, 49 Bianconi, M., Smith, G. P., Haines, C. P., et al. 2018, MNRAS, 473, L79 Biviano, A., \& Katgert, P. 2004, A\&A, 424, 779

Biviano, A., Katgert, P., Mazure, A., et al. 1997, A\&A, 321, 84 Blake, C., Pracy, M. B., Couch, W. J., et al. 2004, MNRAS, 355, 713 Bland-Hawthorn, J., Bryant, J., Robertson, G., et al. 2011, OExpr, 19, 2649 Blanton, M. R., \& Moustakas, J. 2009, ARA\&A, 47, 159

Boselli, A., Cortese, L., Boquien, M., et al. 2014a, A\&A, 564, A67

Boselli, A., Fossati, M., Ferrarese, L., et al. 2018, A\&A, 614, A56

Boselli, A., \& Gavazzi, G. 2006, PASP, 118, 517

Boselli, A., Roehlly, Y., Fossati, M., et al. 2016, A\&A, 596, A11

Boselli, A., Voyer, E., Boissier, S., et al. 2014b, A\&A, 570, A69

Bryan, G. L., \& Norman, M. L. 1998, ApJ, 495, 80

Bryant, J. J., Bland-Hawthorn, J., Fogarty, L. M. R., Lawrence, J. S., \& Croom, S. M. 2014, MNRAS, 438, 869

Bryant, J. J., Bland-Hawthorn, J., Lawrence, J., et al. 2016, Proc. SPIE, 9908 , 99081F

Bryant, J. J., Owers, M. S., Robotham, A. S. G., et al. 2015, MNRAS, 447, 2857

Byrd, G., \& Valtonen, M. 1990, ApJ, 350, 89

Cameron, E. 2011, PASA, 28, 128

Cappellari, M. 2017, MNRAS, 466, 798

Cappellari, M., \& Copin, Y. 2003, MNRAS, 342, 345

Cappellari, M., \& Emsellem, E. 2004, PASP, 116, 138

Cardiel, N., Gorgas, J., Cenarro, J., \& Gonzalez, J. J. 1998, A\&AS, 127, 597

Carter, B. J., Fabricant, D. G., Geller, M. J., Kurtz, M. J., \& McLean, B. 2001, ApJ, 559, 606

Catinella, B., Saintonge, A., Janowiecki, S., et al. 2018, MNRAS, 476, 875

Chilingarian, I. V., Melchior, A.-L., \& Zolotukhin, I. Y. 2010, MNRAS, 405, 1409

Chung, A., van Gorkom, J. H., Kenney, J. D. P., Crowl, H., \& Vollmer, B. 2009a, AJ, 138, 1741

Chung, A., van Gorkom, J. H., Kenney, J. D. P., \& Vollmer, B. 2007, ApJL, 659, L115

Chung, S. M., Gonzalez, A. H., Clowe, D., et al. 2009b, ApJ, 691, 963

Cid Fernandes, R., Pérez, E., García Benito, R., et al. 2013, A\&A, 557, A86

Cid Fernandes, R., Stasińska, G., Mateus, A., \& Vale Asari, N. 2011, MNRAS, 413,1687

Cid Fernandes, R., Stasińska, G., Schlickmann, M. S., et al. 2010, MNRAS, 403, 1036

Colless, M., Dalton, G., Maddox, S., et al. 2001, MNRAS, 328, 1039

Colless, M., \& Dunn, A. M. 1996, ApJ, 458, 435

Cortese, L., Catinella, B., Boissier, S., Boselli, A., \& Heinis, S. 2011, MNRAS, 415, 1797

Cortese, L., Ciesla, L., Boselli, A., et al. 2012, A\&A, 540, A52

Cortese, L., Gavazzi, G., Boselli, A., et al. 2006, A\&A, 453, 847

Cortese, L., \& Hughes, T. M. 2009, MNRAS, 400, 1225

Cortese, L., Marcillac, D., Richard, J., et al. 2007, MNRAS, 376, 157

Couch, W. J., \& Sharples, R. M. 1987, MNRAS, 229, 423

Croom, S. M., Lawrence, J. S., Bland-Hawthorn, J., et al. 2012, MNRAS, 421,872

Crowl, H. H., \& Kenney, J. D. P. 2008, AJ, 136, 1623

Darvish, B., Mobasher, B., Sobral, D., et al. 2016, ApJ, 825, 113

de Carvalho, R. R., Ribeiro, A. L. B., Stalder, D. H., et al. 2017, AJ, 154, 96

de Jong, J. T. A., Verdoes Kleijn, G. A., Boxhoorn, D. R., et al. 2015, A\&A, 582, A62

De Lucia, G., Weinmann, S., Poggianti, B. M., Aragón-Salamanca, A., \& Zaritsky, D. 2012, MNRAS, 423, 1277

Domainko, W., Mair, M., Kapferer, W., et al. 2006, A\&A, 452, 795

Dressler, A. 1980, ApJ, 236, 351

Dressler, A., \& Gunn, J. E. 1983, ApJ, 270, 7

Dressler, A., Smail, I., Poggianti, B. M., et al. 1999, ApJS, 122, 51

Driver, S. P., Hill, D. T., Kelvin, L. S., et al. 2011, MNRAS, 413, 971

Duong, T. 2007, Journal of Statistical Software, 21, 1

Duong, T., Goud, B., \& Schauer, K. 2012, PNAS, 109, 8382

Falcón-Barroso, J., Sánchez-Blázquez, P., Vazdekis, A., et al. 2011, A\&A, 532, A95

Fasano, G., Marmo, C., Varela, J., et al. 2006, A\&A, 445, 805

Ferrarese, L., Côté, P., Cuillandre, J.-C., et al. 2012, ApJS, 200, 4

Fisher, D., Fabricant, D., Franx, M., \& van Dokkum, P. 1998, ApJ, 498, 195

Fogarty, L. M. R., Scott, N., Owers, M. S., et al. 2015, MNRAS, 454, 2050

Fossati, M., Fumagalli, M., Boselli, A., et al. 2016, MNRAS, 455, 2028

Fossati, M., Mendel, J. T., Boselli, A., et al. 2018, A\&A, 614, A57

Fritz, J., Moretti, A., Gullieuszik, M., et al. 2017, ApJ, 848, 132

Fumagalli, M., Fossati, M., Hau, G. K. T., et al. 2014, MNRAS, 445, 4335

Gavazzi, G., Boselli, A., Cortese, L., et al. 2006, A\&A, 446, 839 
Gavazzi, G., Boselli, A., Pedotti, P., Gallazzi, A., \& Carrasco, L. 2002, A\&A, 396, 449

Gavazzi, G., Consolandi, G., Gutierrez, M. L., Boselli, A., \& Yoshida, M. 2018, A\&A, 618, A130

Gavazzi, G., Fumagalli, M., Cucciati, O., \& Boselli, A. 2010, A\&A, 517, A73

Ghigna, S., Moore, B., Governato, F., et al. 1998, MNRAS, 300, 146

Ghirardini, V., Eckert, D., Ettori, S., et al. 2019, A\&A, 621, A41

Gill, S. P. D., Knebe, A., \& Gibson, B. K. 2005, MNRAS, 356, 1327

González Delgado, R. M., Cerviño, M., Martins, L. P., Leitherer, C., \& Hauschildt, P. H. 2005, MNRAS, 357, 945

Green, A. W., Croom, S. M., Scott, N., et al. 2018, MNRAS, 475, 716

Grevesse, N., \& Sauval, A. J. 1998, SSRv, 85, 161

Gullieuszik, M., Poggianti, B. M., Moretti, A., et al. 2017, ApJ, 846, 27

Gunn, J. E., \& Gott, J. R. I. 1972, ApJ, 176, 1

Haines, C. P., Finoguenov, A., Smith, G. P., et al. 2018, MNRAS, 477, 4931

Haines, C. P., Pereira, M. J., Smith, G. P., et al. 2013, ApJ, 775, 126

Haines, C. P., Pereira, M. J., Smith, G. P., et al. 2015, ApJ, 806, 101

Hernández-Fernández, J. D., Haines, C. P., Diaferio, A., et al. 2014, MNRAS, 438,2186

Hill, D. T., Kelvin, L. S., Driver, S. P., et al. 2011, MNRAS, 412, 765

Ho, I.-T., Kewley, L. J., Dopita, M. A., et al. 2014, MNRAS, 444, 3894

Ho, I.-T., Medling, A. M., Groves, B., et al. 2016, Ap\&SS, 361, 280

Hudson, M. J., Stevenson, J. B., Smith, R. J., et al. 2010, MNRAS, 409, 405

Jaffé, Y. L., Poggianti, B. M., Moretti, A., et al. 2018, MNRAS, 476, 4753

Jaffé, Y. L., Smith, R., Candlish, G. N., et al. 2015, MNRAS, 448, 1715

Jung, S. L., Choi, H., Wong, O. I., et al. 2018, ApJ, 865, 156

Kauffmann, G., Heckman, T. M., Tremonti, C., et al. 2003a, MNRAS, 346, 1055

Kauffmann, G., Heckman, T. M., White, S. D. M., et al. 2003b, MNRAS, 341,54

Kauffmann, G., Heckman, T. M., White, S. D. M., et al. 2003c, MNRAS, 341,33

Kelvin, L. S., Driver, S. P., Robotham, A. S. G., et al. 2012, MNRAS, 421, 1007

Kewley, L. J., Dopita, M. A., Sutherland, R. S., Heisler, C. A., \& Trevena, J. 2001, ApJ, 556, 121

Kewley, L. J., Groves, B., Kauffmann, G., \& Heckman, T. 2006, MNRAS, 372, 961

Koopmann, R. A., \& Kenney, J. D. P. 2004a, ApJ, 613, 851

Koopmann, R. A., \& Kenney, J. D. P. 2004b, ApJ, 613, 866

Larson, R. B., Tinsley, B. M., \& Caldwell, C. N. 1980, ApJ, 237, 692

Lee, B., Chung, A., Tonnesen, S., et al. 2017, MNRAS, 466, 1382

Lewis, I., Balogh, M., De Propris, R., et al. 2002, MNRAS, 334, 673

Lindner, R. R., Vera-Ciro, C., Murray, C. E., et al. 2015, AJ, 149, 138

Liske, J., Baldry, I. K., Driver, S. P., et al. 2015, MNRAS, 452, 2087

Lopes, P. A. A., Ribeiro, A. L. B., \& Rembold, S. B. 2017, MNRAS, 472, 409

Mahajan, S., Mamon, G. A., \& Raychaudhury, S. 2011, MNRAS, 416, 2882

Manzer, L. H., \& De Robertis, M. M. 2014, ApJ, 788, 140

Markwardt, C. B. 2009, in ASP Conf. Ser. 411, Astronomical Data Analysis Software and Systems XVIII, ed. D. A. Bohlender, D. Durand, \& P. Dowler (San Francisco, CA: ASP), 251

Marziani, P., D'Onofrio, M., Bettoni, D., et al. 2017, A\&A, 599, A83

McFarland, J. P., Verdoes-Kleijn, G., Sikkema, G., et al. 2013, ExA, 35, 45

McGee, S. L., Balogh, M. L., Bower, R. G., Font, A. S., \& McCarthy, I. G. 2009, MNRAS, 400, 937

Medling, A. M., Cortese, L., Croom, S. M., et al. 2018, MNRAS, 475, 5194 Meert, A., Vikram, V., \& Bernardi, M. 2015, MNRAS, 446, 3943

Merluzzi, P., Busarello, G., Dopita, M. A., et al. 2013, MNRAS, 429, 1747

Merluzzi, P., Busarello, G., Dopita, M. A., et al. 2016, MNRAS, 460, 3345

Miller, C. J., Nichol, R. C., Gómez, P. L., Hopkins, A. M., \& Bernardi, M. 2003, ApJ, 597, 142

Moore, B., Katz, N., Lake, G., Dressler, A., \& Oemler, A. 1996, Natur, 379,613

Moran, S. M., Ellis, R. S., Treu, T., et al. 2007, ApJ, 671, 1503

Moretti, A., Gullieuszik, M., Poggianti, B., et al. 2017, A\&A, 599, A81

Muzzin, A., van der Burg, R. F. J., McGee, S. L., et al. 2014, ApJ, 796, 65

Muzzin, A., Wilson, G., Yee, H. K. C., et al. 2012, ApJ, 746, 188

Noble, A. G., Muzzin, A., McDonald, M., et al. 2019, ApJ, 870, 56

Noble, A. G., Webb, T. M. A., Muzzin, A., et al. 2013, ApJ, 768, 118
Nulsen, P. E. J. 1982, MNRAS, 198, 1007

Oman, K. A., \& Hudson, M. J. 2016, MNRAS, 463, 3083

Oman, K. A., Hudson, M. J., \& Behroozi, P. S. 2013, MNRAS, 431, 2307

Owers, M. S., Allen, J. T., Baldry, I., et al. 2017, MNRAS, 468, 1824

Paccagnella, A., Vulcani, B., Poggianti, B. M., et al. 2016, ApJL, 816, L25

Paccagnella, A., Vulcani, B., Poggianti, B. M., et al. 2017, ApJ, 838, 148

Peacock, J. A. 1983, MNRAS, 202, 615

Peng, Y.-J., Lilly, S. J., Kovač, K., et al. 2010, ApJ, 721, 193

Poggianti, B. M., Aragón-Salamanca, A., Zaritsky, D., et al. 2009, ApJ, 693, 112

Poggianti, B. M., Bridges, T. J., Komiyama, Y., et al. 2004, ApJ, 601, 197

Poggianti, B. M., Moretti, A., Gullieuszik, M., et al. 2017, ApJ, 844, 48

Poggianti, B. M., Smail, I., Dressler, A., et al. 1999, ApJ, 518, 576

Pracy, M. B., Couch, W. J., Blake, C., et al. 2005, MNRAS, 359, 1421

Pracy, M. B., Kuntschner, H., Couch, W. J., et al. 2009, MNRAS, 396, 1349

Pracy, M. B., Owers, M. S., Zwaan, M., et al. 2014, MNRAS, 443, 388

Prada, F., Klypin, A. A., Cuesta, A. J., Betancort-Rijo, J. E., \& Primack, J. 2012, MNRAS, 423, 3018

Quilis, V., Moore, B., \& Bower, R. 2000, Sci, 288, 1617

Reiprich, T. H., Basu, K., Ettori, S., et al. 2013, SSRv, 177, 195

Rhee, J., Smith, R., Choi, H., et al. 2017, ApJ, 843, 128

Robotham, A. S. G., Davies, L. J. M., Driver, S. P., et al. 2018, MNRAS, 476, 3137

Robotham, A. S. G., Norberg, P., Driver, S. P., et al. 2011, MNRAS, 416, 2640

Robotham, A. S. G., \& Obreschkow, D. 2015, PASA, 32, e033

Robotham, A. S. G., Taranu, D. S., Tobar, R., Moffett, A., \& Driver, S. P 2017, MNRAS, 466, 1513

Roediger, E. 2009, AN, 330, 888

Roediger, E., \& Brüggen, M. 2006, MNRAS, 369, 567

Roediger, E., \& Brüggen, M. 2007, MNRAS, 380, 1399

Rola, C., \& Pelat, D. 1994, A\&A, 287, 676

Saunders, W., Bridges, T., Gillingham, P., et al. 2004, Proc. SPIE, 5492, 389

Schaefer, A. L., Croom, S. M., Allen, J. T., et al. 2017, MNRAS, 464, 121

Schaefer, A. L., Croom, S. M., Scott, N., et al. 2019, MNRAS, 483, 2851

Schulz, S., \& Struck, C. 2001, MNRAS, 328, 185

Scott, N., van de Sande, J., Croom, S. M., et al. 2018, MNRAS, 481, 2299

Seibert, M., Wyder, T., Neill, J., et al. 2012, AAS Meeting, 219, 340.01

Shanks, T., Metcalfe, N., Chehade, B., et al. 2015, MNRAS, 451, 4238

Sharp, R., Allen, J. T., Fogarty, L. M. R., et al. 2015, MNRAS, 446, 1551

Sharp, R., Saunders, W., Smith, G., et al. 2006, Proc. SPIE, 6269, 62690G

Singh, R., van de Ven, G., Jahnke, K., et al. 2013, A\&A, 558, A43

Smith, R. J., Lucey, J. R., Hammer, D., et al. 2010, MNRAS, 408, 1417

Solanes, J. M., Manrique, A., García-Gómez, C., et al. 2001, ApJ, 548, 97

Taranu, D. S., Hudson, M. J., Balogh, M. L., et al. 2014, MNRAS, 440, 1934

Taylor, E. N., Hopkins, A. M., Baldry, I. K., et al. 2011, MNRAS, 418, 1587

Tran, K.-V. H., Franx, M., Illingworth, G., Kelson, D. D., \& van Dokkum, P. 2003, ApJ, 599, 865

van de Sande, J., Bland-Hawthorn, J., Fogarty, L. M. R., et al. 2017, ApJ, 835,104

van der Burg, R. F. J., McGee, S., Aussel, H., et al. 2018, A\&A, 618, A140

van der Marel, R. P., \& Franx, M. 1993, ApJ, 407, 525

Vazdekis, A., Sánchez-Blázquez, P., Falcón-Barroso, J., et al. 2010, MNRAS, 404, 1639

Veilleux, S., \& Osterbrock, D. E. 1987, ApJS, 63, 295

Vikhlinin, A., Kravtsov, A., Forman, W., et al. 2006, ApJ, 640, 691

Vollmer, B., Cayatte, V., Balkowski, C., \& Duschl, W. J. 2001, ApJ, 561, 708

von der Linden, A., Wild, V., Kauffmann, G., White, S. D. M., \& Weinmann, S. 2010, MNRAS, 404, 1231

Wetzel, A. R., Tinker, J. L., \& Conroy, C. 2012, MNRAS, 424, 232

Wetzel, A. R., Tinker, J. L., Conroy, C., \& van den Bosch, F. C. 2013, MNRAS, 432, 336

Worthey, G., Faber, S. M., Gonzalez, J. J., \& Burstein, D. 1994, ApJS, 94, 687 Worthey, G., \& Ottaviani, D. L. 1997, ApJS, 111, 377

Wright, A. H., Robotham, A. S. G., Bourne, N., et al. 2016, MNRAS, 460, 765

Yagi, M., Yoshida, M., Komiyama, Y., et al. 2010, AJ, 140, 1814

Yan, R., Newman, J. A., Faber, S. M., et al. 2006, ApJ, 648, 281

Yang, Y., Zabludoff, A. I., Zaritsky, D., \& Mihos, J. C. 2008, ApJ, 688, 945

York, D. G., Adelman, J., Anderson, J. E., Jr., et al. 2000, AJ, 120, 1579

Zabludoff, A. I., Zaritsky, D., Lin, H., et al. 1996, ApJ, 466, 104 